\title{
Understanding interactive multidimensional projections
}



Assinatura:

\title{
Samuel Gomes Fadel
}

\section{Understanding interactive multidimensional projections}

\begin{abstract}
Master dissertation submitted to the Instituto de Ciências Matemáticas e de Computação - ICMCUSP, in partial fulfillment of the requirements for the degrees of the Master Program in Computer Science and Computational Mathematics. FINAL VERSION

Concentration Area: Computer Science and Computational Mathematics
\end{abstract}

Advisor: Prof. Dr. Fernando Vieira Paulovich 
Ficha catalográfica elaborada pela Biblioteca Prof. Achille Bassi e Seção Técnica de Informática, ICMC/USP, com os dados fornecidos pelo(a) autor(a)

Fadel, Samuel Gomes
Understanding interactive multidimensional
projections / Samuel Gomes Fadel; orientador Fernando
Vieira Paulovich. - São Carlos - SP, 2016.
88 p.
Dissertação (Mestrado - Programa de Pós-Graduação
em Ciências de Computação e Matemática Computacional)
- Instituto de Ciências Matemáticas e de Computação,
Universidade de São Paulo, 2016.
1. information visualization. 2. dimensionality
reduction. I. Paulovich, Fernando Vieira, orient. II.
Título.


Samuel Gomes Fadel

\title{
Compreendendo projeções multidimensionais interativas
}

\author{
Dissertação apresentada ao Instituto de Ciências \\ Matemáticas e de Computação - ICMC-USP, como \\ parte dos requisitos para obtenção dos títulos \\ de Mestre em Ciências - Ciências de Computação e \\ Matemática Computacional. VERSÃO REVISADA \\ Área de Concentração: Ciências de Computação e \\ Matemática Computacional \\ Orientador: Prof. Dr. Fernando Vieira Paulovich
}

USP - São Carlos

Dezembro de 2016 



\section{ACKNOWLEDGEMENTS}

First and foremost, I would like to thank my advisor Fernando Paulovich for his guidance, patience and interesting discussions (either scientific or philosophical). You introduced me to the world of scientific research and showed me how challenging, fun and satisfying it can be. In addition, I would like to thank professor Alex Telea from the University of Groningen for his time, really neat ideas, and guidance. You are truly an inspiration for any researcher.

In alphabetical order and hopefully not forgetting any names, I would also like to thank all my friends and colleagues that made the period during this research more pleasant: André Perina, Antonio Canabrava, Bianca Filtre, Bruno Tasso, Carlos Zampieri, Danilo Coimbra, Felipe Duarte, Filipe Perez, Filipe Verri, Francisco Fatore, Fábio Sikansi, Gabriel de Barros, Jorge Ono, Karina Emboaba, Lucas Sodré, Marcelo Brasileiro, Paulo Rauber, Renato Rodrigues, Rodolfo Cunha, Rodrigo Bataglioli, Stefânia Rodrigues, Tiago Nazaré, Tácito Neves, Verivaldo Lobo.

I am thankful to my family for their unconditional support, encouragement and love. You made all of this possible in the first place.

Last but not least, Flávia, I am forever grateful for your unconditional and unrelenting support, patience and love.

Disclaimer: This research was supported by FAPESP (grant number 2014/13010-7), CAPES and CNPq. The opinions, assumptions and conclusions or recommendations expressed in this material are those of the authors and do not necessarily reflect the views of FAPESP, CAPES nor CNPq. 



\section{ABSTRACT}

FADEL, S. G.. Understanding interactive multidimensional projections. 2016. 88 f. Master dissertation (Master student Program in Computer Science and Computational Mathematics) Instituto de Ciências Matemáticas e de Computação (ICMC/USP), São Carlos - SP.

The large amount of available data on a diverse range of human activities provides many opportunities for understanding, improving and revealing unknown patterns in them. Powerful automatic methods for extracting this knowledge from data are already available from machine learning and data mining. They, however, rely on the expertise of analysts to improve their results when those are not satisfactory. In this context, interactive multidimensional projections are a useful tool for the analysis of multidimensional data by revealing their underlying structure while allowing the user to manipulate the results to provide further insight into this structure. This manipulation, however, has received little attention regarding their influence on the mappings, as they can change the final layout in unpredictable ways. This is the main motivation for this research: understanding the effects caused by changes in these mappings. We approach this problem from two perspectives. First, the user perspective, we designed and developed visualizations that help reduce the trial and error in this process by providing the right piece of information for performing manipulations. Furthermore, these visualizations help explain the changes in the map caused by such manipulations. Second, we defined the effectiveness of manipulation in quantitative terms, then developed an experimental framework for assessing manipulations in multidimensional projections under this view. This framework is based on improving mappings using known evaluation measures for these techniques. Using the improvement of measures as different types of manipulations, we perform a series of experiments on five datasets, five measures, and four techniques. Our experimental results show that there are possible types of manipulations that can happen effectively, with some techniques being more susceptible to manipulations than others.

Key-words: information visualization, dimensionality reduction. 



\section{RESUMO}

FADEL, S. G.. Understanding interactive multidimensional projections. 2016. 88 f. Master dissertation (Master student Program in Computer Science and Computational Mathematics) Instituto de Ciências Matemáticas e de Computação (ICMC/USP), São Carlos - SP.

O grande volume de dados disponíveis em uma diversa gama de atividades humanas cria várias oportunidades para entendermos, melhorarmos e revelarmos padrões previamente desconhecidos em tais atividades. Métodos automáticos para extrair esses conhecimentos a partir de dados já existem em áreas como aprendizado de máquina e mineração de dados. Entretanto, eles dependem da perícia do analista para obter melhores resultados quando estes não são satisfatórios. Neste contexto, técnicas de projeção multidimensional interativas são uma ferramenta útil para a análise de dados multidimensionais, revelando sua estrutura subjacente ao mesmo tempo que permite ao analista manipular os resultados interativamente, estendendo o processo de exploração. Essa interação, entretanto, não foi estudada com profundidade com respeito à sua real influência nos mapeamentos, já que podem causar mudanças não esperadas no mapeamento final. Essa é a principal motivação desta pesquisa: entender os efeitos causados pelas mudanças em tais mapeamentos. Abordamos o problema de duas perspectivas. Primeiro, da perspectiva do usuário, desenvolvemos visualizações que ajudam a diminuir tentativas e erros neste processo provendo a informação necessária a cada passo da interação. Além disso, essas visualizações ajudam a explicar as mudanças causadas no mapeamento pela manipulação. A segunda perspectiva é a efetividade da manipulação. Definimos de forma quantitativa a efetividade da manipulação, e então desenvolvemos um arcabouço para avaliar manipulações sob a visão da efetividade. Este arcabouço é baseado em melhorias nos mapeamentos usando medidas de avaliação conhecidas para tais técnicas. Usando tais melhorias como diferentes formas de manipulação, realizamos uma série de experimentos em cinco bases de dados, cinco medidas e quatro técnicas. Nossos resultados experimentais nos dão evidências que existem certos tipos de manipulação que podem acontecer efetivamente, com algumas técnicas sendo mais suscetíveis a manipulações do que outras.

Palavras-chave: visualização de informação, redução de dimensionalidade. 

Figure 1 - An overview of PCA. . . . . . . . . . . . 23

Figure 2 - The information retrieval task in information visualization, as introduced by Venna et al. (2010). . . . . . . . . . . . . . . . . . . . 31

Figure 3 - An example of interaction in Dis-function. . . . . . . . . . . . . 37

Figure 4 - The image retrieval system prototype by Mamani et al. (2013). . . . . . . . 39

Figure 5 - Illustration of a three-dimensional grand tour path. . . . . . . . . . . 40

Figure 6 - The transition between two scatterplots in the scatterplot matrix navigation framework proposed by Elmqvist, Dragicevic e Fekete (2008). . . . . . . . 41

Figure 7 - The different ways of cell navigation in the scatterplot matrix navigation framework proposed by Elmqvist, Dragicevic e Fekete (2008). . . . . . . . 42

Figure 8 - Highlighting regions of interest with the technique described by Martins et al. (2014). . . . . . . . . . . . . . . . . . . . 4 47

Figure 9 - Selection of control points using influence as a guide. . . . . . . . . . 48

Figure 10 - Brushing and linking with the additional ordered bar plot allows more complex, informed selections and inspection. . . . . . . . . . . 50

Figure 11 - Illustration of the graph bundling technique employed in the identifying unexpected behavior when manipulating. . . . . . . . . . . 51

Figure 12 - Difference in $e^{\text {aggr }}$ relative to previous map. . . . . . . . . . . . 52

Figure 13 - Illustration of the animation interaction mechanism for visualizing changes in the map caused by manipulation. . . . . . . . . . . . . . . 53

Figure 14 - Overview of the methodology for effectiveness analysis. . . . . . . . . . 60

Figure 15 - Details of how we present our results for many sets of control points. . . . . 60

Figure 16 - Details of how we present our results for a set of control points chosen using the $k$-medoids strategy. . . . . . . . . . . . . . . . 61

Figure 17 - An example of automated manipulation (NEWSGROUPS dataset; see chapter 5 for details) . . . . . . . . . . . . . . . . . . . . . 63

Figure 18 - Scatterplots of the average measurements over all control point selections, before and after manipulation. . . . . . . . . . . . .

Figure 19 - Line plots of $95 \%$ confidence intervals for the difference between a measure after manipulation and before. . . . . . . . . . . . . 72 
Figure 20 - The improvement in measures both in $\mathscr{Y}^{\prime}$ and $\mathscr{Y}$, starting from a randomly projected $\mathscr{Y}^{\prime}$ and then moving towards an improved placement according to a measure. . . . . . . . . . . . . . . . . . . . . 73 


\section{LIST OF TABLES}

Table 1 - Summary of the datasets used in our experiments for evaluating effectiveness in ascending order of number of instances. . . . . . . . . . . . . . . . 66 

INTRODUCTION .................... 17

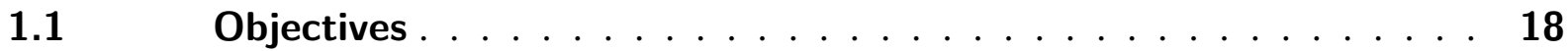

$1.2 \quad$ Structure of the document $\ldots \ldots \ldots \ldots$

2 BACKGROUND AND RELATED WORK . . . . . . . . . . . . 21

$2.1 \quad$ Automatic methods and techniques . . . . . . . . . . . 22

2.1.1 Principal Component Analysis . . . . . . . . . . . . . . . . . 22

2.1.2 Classical Scaling . . . . . . . . . . . . . . . . . 24

2.1.3 Projection Pursuit . . . . . . . . . . . . . . . . . 26

2.1.4 Sammon's Mapping . . . . . . . . . . . . . . . . 27

2.1.5 Force-based point placement . . . . . . . . . . . . 28

2.1.6 Stochastic Neighbor Embedding . . . . . . . . . . . . . . . 30

$2.2 \quad$ Interactive methods and techniques . . . . . . . . . . 32

2.2.1 Least Squares Projection . . . . . . . . . . . . . . . . . . 32

2.2.2 Part Linear Multidimensional Projection . . . . . . . . . . . . . . 33

2.2.3 Local Affine Multidimensional Projection . . . . . . . . . . . . . . . . 34

$2.3 \quad$ Interactive data transformation . . . . . . . . . . . . 36

2.3.1 Dis-function . . . . . . . . . . . . . . . . 36

2.3.2 User-driven feature space transformation . . . . . . . . . . . . 38

$2.4 \quad$ Animation and transition-based projections . . . . . . . . . 39

2.4.1 Grand Tour . . . . . . . . . . . . . . . . . . . . 39

2.4.2 Scatterplot matrix navigation . . . . . . . . . . . . . . . 41

$2.5 \quad$ Chapter conclusions . . . . . . . . . . . . . . 42

3 VISUALLY UNDERSTANDING MANIPULATION . . . . . . 45

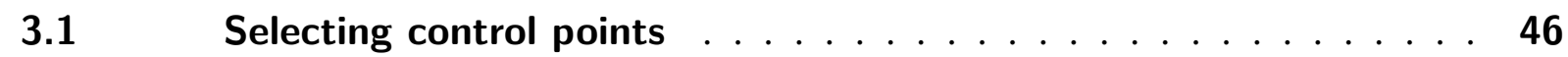

3.1.1 Regions of interest . . . . . . . . . . . . . . 46

3.1.2 Precise selections, brushing and linking . . . . . . . . . . 48

$3.2 \quad$ Highlighting unexpected behavior ............. 49

$3.3 \quad$ Explaining the manipulation . . . . . . . . . . . 51

3.3.1 Changes in metrics . . . . . . . . . . . . . . . . 51

3.3.2 Changes in positioning ................ 52 


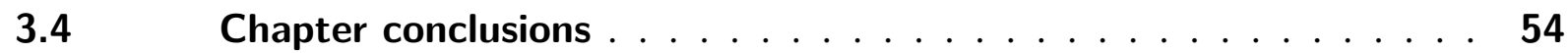

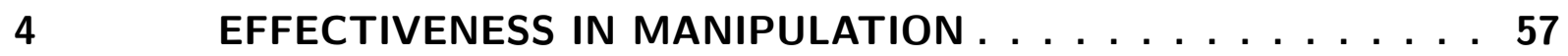

$4.1 \quad$ Defining and measuring effectiveness $\ldots \ldots \ldots \ldots \ldots$

$4.2 \quad$ Methodology . . . . . . . . . . . . . . . . . . 59

4.2.1 Quality measures . . . . . . . . . . . . . . . . . . . . 61

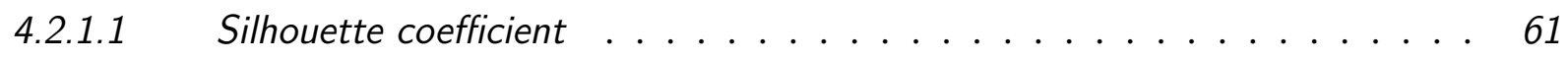

4.2.1.2 Sammon's stress . . . . . . . . . . . . . . . . . . . . . . . . 62

4.2.1.3 Neighborhood preservation index . . . . . . . . . . . . . . . . 62

4.2.1.4 Smoothed precision and smoothed recall . . . . . . . . . . . . . . 62

4.2.2 Automated manipulation procedures . . . . . . . . . . . . . 63

$4.3 \quad$ Chapter conclusions . . . . . . . . . . . . . . . . . . . . 64

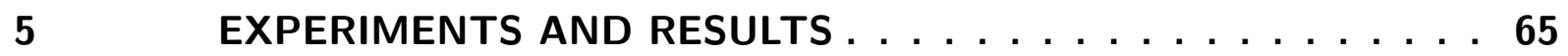

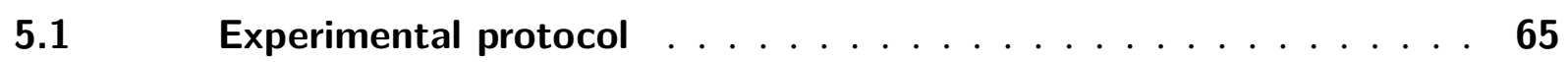

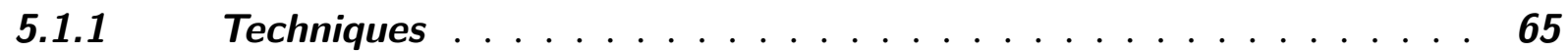

5.1.2 Datasets . . . . . . . . . . . . . . . . . 66

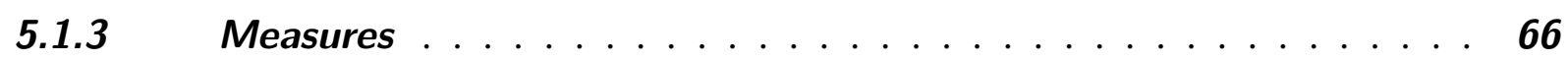

5.1.4 Control point selection and mapping . . . . . . . . . . . 67

$5.2 \quad$ Results and discussion . . . . . . . . . . . . . . 67

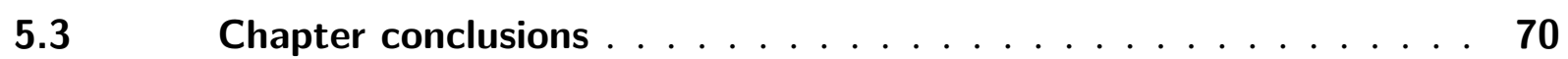

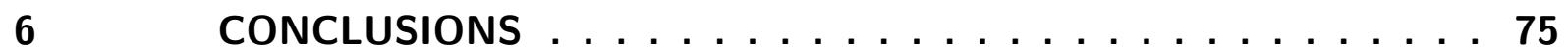

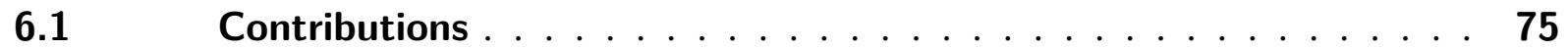

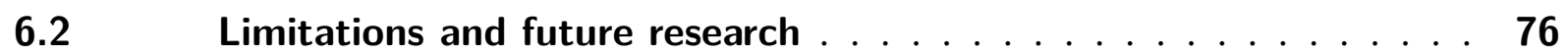

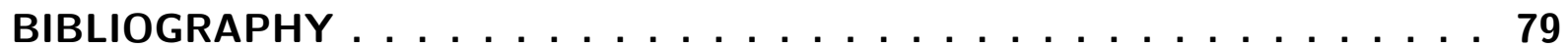

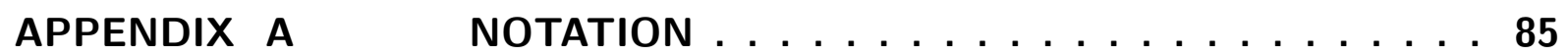

APPENDIX B DATA PREPARATION AND PREPROCESSING . . 87 
The ever growing data collection and storage provides many opportunities for understanding, improving and discovering the unexpected in many areas of human activities. Specifically, machine learning, data mining and visualization have become crucial in making sense of, obtaining insights, and deriving knowledge from this data. Although the first two already provide powerful methods for automatically modeling and analyzing data, they rely on the experience of the analyst in order to improve their results. In this context, data visualization complements the analysis via intuitive mechanisms for interacting with data, providing analysts with a tool for guiding the refinement and tuning of machine learning and data mining methods (KEIM et al., 2010; SUN et al., 2013).

This coordinated use of automatic analysis and visualization is known as visual analytics. It "combines the automated analysis techniques with interactive visualizations for an effective understanding, reasoning and decision making on the basis of very large datasets" (KEIM et al., 2010). One specific aspect of visual analytics is the use of interactive interfaces in the visual analysis, since they enable repeated explorations while analysts develop insights about patterns and relationships in the data (HEER; SHNEIDERMAN, 2012).

A particular class of visualization techniques, called multidimensional projections, has attracted attention in the information visualization community for some time, mainly because of their broad applicability (PAULOVICH et al., 2008). As the name suggests, these techniques create visual representations for multidimensional data, that is, data where items with $p \geq 4$ attributes represent points in a $p$-dimensional space (SHNEIDERMAN, 1996). This is done by reducing the number of attributes to 2 or 3 while still retaining as much of the original data structure as possible. The new reduced data is then displayed as a scatterplot, where each item is represented as a glyph.

Multidimensional projections have been successfully applied in many different areas, such as vector field analysis (II et al., 2010), visual text mining (CHEN et al., 2009; WISE, 1999; 
ANDREWS et al., 2002), and biosensor analysis (PAULOVICH et al., 2011b; PAULOVICH et al., 2011). Recent research on visualization of multidimensional data gave evidence that interactive methods can deal with complex data and overcome limitations of previous fully automatic methods (JOIA et al., 2011; PAULOVICH; SILVA; NONATO, 2012; MAMANI et al., 2013).

An example of these advantages is one particular application proposed by Mamani et al. (2013) that involves incorporating user knowledge via multidimensional projections in order to transform the original data. This application is used for retrieving similar images from a collection. It exposes a subsample of this collection to the user. A visual representation of those images is then created using a multidimensional projection technique, positioning thumbnails of each image in this subsample according to their content. Later, users must then group similar images, as dictated by their own judgement, by dragging those thumbnails. Finally, the system transforms the original data features in order to reflect the changes made by the user, effectively improving the retrieval of similar images.

This use of interactivity fully exploits the capabilities of multidimensional projections in representing groups of instances in the data. By taking advantage of an intuitive way of implicitly defining the similarity between images, the user can directly see what should be done to achieve the desired grouping. As all interactive multidimensional projection techniques (PEKALSKA et al., 1999; PAULOVICH et al., 2008; PAULOVICH; SILVA; NONATO, 2010; JOIA et al., 2011), however, the system does not impose any kind of restrictions to the subsample manipulation. The user, by introducing knowledge external to the provided data via manipulation, distorts the final mapping with information not previously observed. To the best of our knowledge, there are no studies regarding the distortion caused by these manipulations.

Given the relevance of visual analytics as a data analysis discipline, these issues are significant gaps in the formulation of such techniques. While making use of them, one must be able to assess how that manipulation affects the final visualization while still being able to tell how faithful it is to the original data. As of this writing, no studies have been conducted to properly assess whether interaction in such techniques is significant in providing better representations of data. Moreover, from the user perspective, little to no information is available to guide such interactions, often resulting in a trial-and-error process.

\subsection{Objectives}

Under the hypothesis that properly evaluating and measuring the influence of user manipulation can lead to better incorporation of user knowledge and motivated by the aforementioned limitations on interactive multidimensional projections, the objectives of this research are:

- to provide evidence on whether user interaction can effectively lead to better representa- 
tions of data using interactive multidimensional projections;

- to employ visualization techniques aimed at conveying the distortion introduced by such manipulations in order to provide a better understanding of these interactions.

\subsection{Structure of the document}

The remainder of this document is organized as follows:

- Chapter 2 presents and discusses the technical background and previous work related to the topic of this research;

- Chapter 3 outlines and discusses the visualization techniques for guiding user manipulation, aiming to provide better insights during interaction, reducing trial-and-error;

- Chapter 4 describes the method for quantitatively evaluating effectiveness in user manipulation;

- Chapter 5 presents experimental results of the methods and techniques described in Chapter 4;

- Chapter 6 highlights the contributions and limitations of this research, along with some directions of future work. 



\section{BACKGROUND AND RELATED WORK}

Let $\mathscr{X}=\left\{\mathbf{x}_{1}, \mathbf{x}_{2}, \ldots, \mathbf{x}_{n}\right\}$ be a set of $p$-tuples $\mathbf{x}_{i} \in \mathbb{R}^{p}$ representing $n$ observations of $p>3$ variables. Also consider an analogous representation, which places observations in a matrix $X_{n \times p}$, with each observation occupying a row and each observed variable having a corresponding column. We say that $\mathscr{X}$ (or $X$ ) is a dataset or a set of multidimensional data. Additionally, we call each tuple $\mathbf{x}_{i}$ (or row of $X$ ) a datum, instance or sample, each observed variable (or column of $X$ ) an attribute, feature or dimension. Most of the notation used throughout the text is summarized in Appendix A.

In this chapter, we discuss the problem of exploring multidimensional data by transforming each observation into a low-dimensional representation, where a simple and straightforward visual mapping is possible by using a scatterplot of glyphs. According to Keim (2002), techniques which find such transformation are categorized as Geometrically transformed displays. We say that $\mathbf{y}_{i} \in \mathscr{Y}$ is the low-dimensional representation of $\mathbf{x}_{i}$, with $\mathscr{Y} \subset \mathbb{R}^{q}$ and $q \in\{2,3\}$. We also call $\mathscr{Y}$ a map or, as a slight abuse of terminology, a multidimensional projection or projection of $\mathscr{X}$. When detailing the techniques in this chapter, we mostly consider the setting where $q=2$ without loss of generality.

When visualizing multidimensional data, one is interested in finding patterns, clusters, correlations among pairs of variables, gaps and outliers (SHNEIDERMAN, 1996). By providing mappings that represent some aspect of the original data in a low-dimensional space, it is possible to visually convey these aspects by taking advantage of the human visual system. In this context, we present relevant methods and techniques that were devised or later re-purposed for performing these tasks. They are organized in the following sections: section 2.1 discusses fully automatic methods, which require no input beyond the dataset being analyzed; section 2.2 presents methods that enable user interactivity to influence the resulting visualization; section 2.3 focuses on interactive data transformation via user interactions with scatterplots; section 2.4 presents the use of projections in animations to explore datasets in various different perspectives. 


\subsection{Automatic methods and techniques}

Automatic methods and techniques for visualizing multidimensional data are much more numerous and diverse than their interactive counterparts. The great amount of dimensionality reduction techniques is probably one of the main reasons for that. Thus, we discuss only the most relevant work related to the topic of this research.

Representing multidimensional data as points in a low-dimensional space resembles closely the problem of dimensionality reduction. That is, when mapping this kind of data to a low-dimensional space, one is interested in using fewer dimensions to describe the original data. As it turns out, many dimensionality reduction methods can also be applied to the visualization of multidimensional data when the number of resulting dimensions is adequate. There are two main types of dimensionality reduction: feature selection and feature extraction (PUDIL; HOVOVICOVA, 1998).

Feature selection, also known as feature subset selection, encompasses methods for reducing the dimensionality of data by selecting a subset of the original features with the purpose of retaining only the most useful ones, based on the hypothesis that the original set of features contain redundant and irrelevant features (GUYON; ELISSEEFF, 2003). As this generally does not result in a number of dimensions as few as two or three, these are not part of this review. This does not, however, exclude the use of these methods at a preprocessing stage for data analysis.

Feature extraction techniques, on the other hand, assume that despite having a dimensionality $p$, much of the data lies on a manifold of lower dimensionality. Specifically, this manifold, possibly consisting of a number of disconnected sub-manifolds, has a dimensionality $q$ usually much smaller than $p$ (MAATEN; POSTMA; HERIK, 2009). These techniques attempt to reduce the data dimensionality by transforming the original dataset $\mathscr{X}$ with $p$ dimensions into a dataset $\mathscr{Y}$ with $q$ dimensions while preserving some property of the data. These characteristics makes them similar to methods designed specifically for visualization, therefore we make no distinction between them for the purposes here considered.

\subsubsection{Principal Component Analysis}

According to Jolliffe (2005), principal component analysis (PCA) is probably the most widely used dimensionality reduction technique. Essentially, PCA finds orthogonal directions, called principal components, that describe the original data by maximizing the variance in each direction. This is done in such a way that the first component is the direction of largest variance, the second component is the direction of largest variance orthogonal to the first component, and so on. An overview of the method is presented in Figure 1. As an example, consider the dataset illustrated in Figure 1a, consisting of 500 observations of two variables. The principal components of this dataset are highlighted in a "zoomed in" view of the dataset, shown in Figure 1b. Lastly, the projection onto the principal components is shown in Figure 1c. 
Figure 1 - An overview of PCA. (a) A dataset consisting of 500 observations of two variables: "Var1" and "Var2". (b) The principal components represent the directions of largest variance, with their length scaled to convey the variance in each direction. (c) The data projected on the principal components "PC1" and "PC2".

(a)

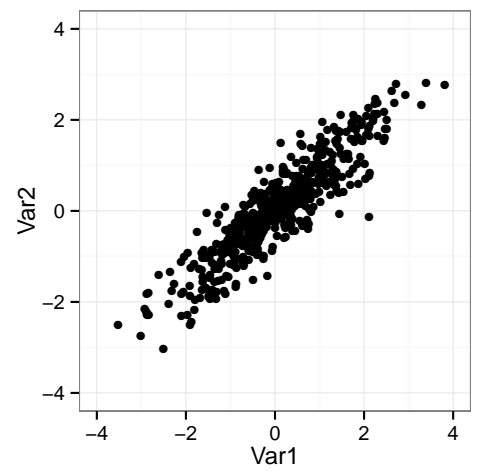

(b)

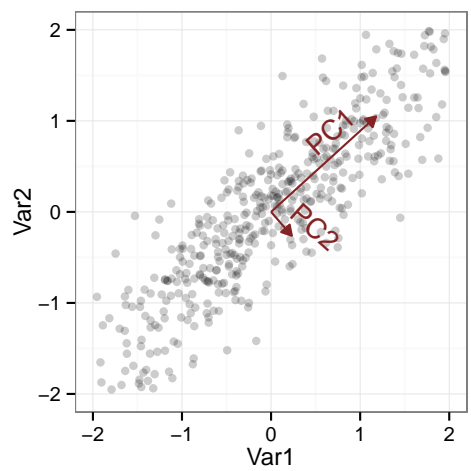

(c)

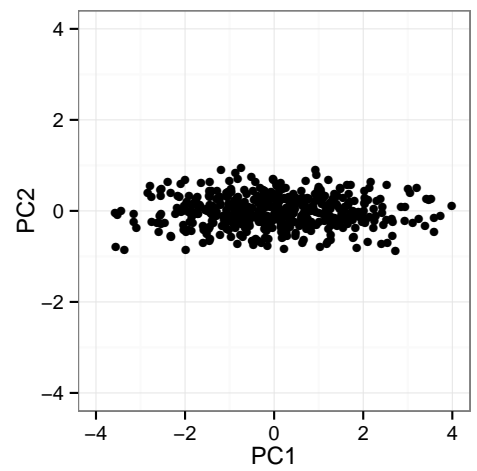

Source: Elaborated by the author.

Mathematically, the principal components $\mathbf{a}_{1}, \mathbf{a}_{2}, \ldots, \mathbf{a}_{p}$ of a dataset $X$ are, in fact, the solution of the following problem

$$
\begin{array}{ll}
\operatorname{maximize} & \operatorname{tr}\left(A^{T} \operatorname{cov}(X) A\right) \\
\text { subject to } & \left\|\mathbf{a}_{i}\right\|^{2}=1 ; \text { for each column } \mathbf{a}_{i} \text { of } A
\end{array}
$$

where $\operatorname{tr}(\cdot)$ denotes the trace and $\operatorname{cov}(X)$ is the sample covariance matrix of $X$. The solution of (2.1) is given by the eigenvectors of $S=\operatorname{cov}(X)$, where $\mathbf{a}_{i}$ is the eigenvector with the $i$ th largest associated eigenvalue, for all $i=1,2, \ldots, p$. While each principal component $\mathbf{a}_{i}$ represents one direction of data variability, its associated eigenvalue represents how large is that variability (JOLLIFFE, 2005; MAATEN; POSTMA; HERIK, 2009).

Accordingly, the components $\mathbf{a}_{1}, \mathbf{a}_{2}, \ldots, \mathbf{a}_{q}$ represent the $q$-dimensional subspace where the data has the largest variability. Therefore, if we are interested in reducing the dimensionality of the dataset $X$ regarding the variability, a projection matrix with only these $q$ column vectors is used to project $X$ into the subspace spanned by them with

$$
Y=X\left[\begin{array}{llll}
\mathbf{a}_{1} & \mathbf{a}_{2} & \cdots & \mathbf{a}_{q}
\end{array}\right]
$$

Unsurprisingly, the transformation given by Equation (2.2) incurs in loss of information, as $q$ might not be high enough to represent the original dataset faithfully. Added to this, data variability might not be the only aspect worth looking for in the data. Consequently, other properties of the data should be also considered in the analysis, as discussed in the following sections. 


\subsubsection{Classical Scaling}

Closely related to PCA is one of the oldest multidimensional scaling (MDS) methods, commonly known as classical scaling (COX; COX, 2000). Multidimensional scaling comprises a family of techniques for solving the problem of finding a low-dimensional space, usually Euclidean, in which to embed a given set of objects described by their dissimilarities ${ }^{1}$ into a set of points in this space, such that the distance between every pair of points $i$ and $j$ are as close as possible to the dissimilarity between objects $i$ and $j$.

Suppose a set of objects for which a dissimilarity measure is given, with $\delta_{i j} \in \mathbb{R}_{0}^{+}$representing the dissimilarity between objects $i$ and $j$. In our setting, $\delta_{i j}$ represents the dissimilarity between tuples $\mathbf{x}_{i}$ and $\mathbf{x}_{j}$ of $\mathscr{X}$ and the low-dimensional space is assumed to have dimensionality $q$. Classical scaling originated as an MDS solution when the dissimilarities are assumed to be Euclidean distances. Specifically, an inner product matrix $B$, with $[B]_{i j}=\mathbf{x}_{i} \cdot \mathbf{x}_{j}$, is built from the given Euclidean distances

$$
d_{i j}^{2}=\left(\mathbf{x}_{i}-\mathbf{x}_{j}\right) \cdot\left(\mathbf{x}_{i}-\mathbf{x}_{j}\right)
$$

and the coordinates of the embedded points are recovered from $B$.

An important aspect of MDS is that relative distances are not enough to determine unique embeddings because they are rotation, translation and reflection invariant. Hence, at first, we assume the data is translated such that the centroid $\overline{\mathbf{x}}$ is placed at the origin, i.e.,

$$
\mathbf{x}_{i} \leftarrow \mathbf{x}_{i}-\overline{\mathbf{x}} \quad \text { for } i=1,2, \ldots, n, \quad \text { with } \overline{\mathbf{x}}=\frac{1}{n} \sum_{k=1}^{n} \mathbf{x}_{k} .
$$

To calculate $B$ from the distances defined by Equation (2.3), we rewrite them as

$$
d_{i j}^{2}=\mathbf{x}_{i} \cdot \mathbf{x}_{i}+\mathbf{x}_{j} \cdot \mathbf{x}_{j}-2 \mathbf{x}_{i} \cdot \mathbf{x}_{j}
$$

and since we assume the data is zero-mean, we have

$$
\begin{aligned}
\frac{1}{n} \sum_{i=1}^{n} d_{i j}^{2} & =\mathbf{x}_{j} \cdot \mathbf{x}_{j}+\frac{1}{n} \sum_{i=1}^{n} \mathbf{x}_{i} \cdot \mathbf{x}_{i} \\
\frac{1}{n} \sum_{j=1}^{n} d_{i j}^{2} & =\mathbf{x}_{i} \cdot \mathbf{x}_{i}+\frac{1}{n} \sum_{j=1}^{n} \mathbf{x}_{j} \cdot \mathbf{x}_{j} \\
\frac{1}{n^{2}} \sum_{i=1}^{n} \sum_{j=1}^{n} d_{i j}^{2} & =\frac{2}{n} \sum_{i=1}^{n} \mathbf{x}_{i} \cdot \mathbf{x}_{i}
\end{aligned}
$$

Substituting (2.6) into (2.5) results in

$$
\begin{aligned}
{[B]_{i j} } & =\mathbf{x}_{i} \cdot \mathbf{x}_{j}, \\
& =-\frac{1}{2}\left(d_{i j}^{2}-\frac{1}{n} \sum_{k=1}^{n} d_{i k}^{2}-\frac{1}{n} \sum_{k=1}^{n} d_{k j}^{2}+\frac{1}{n^{2}} \sum_{k=1}^{n} \sum_{l=1}^{n} d_{k l}^{2}\right) .
\end{aligned}
$$

1 Dissimilarity is a measure of "proximity" between two objects; larger values represent objects that are less similar and therefore further away from each other in some sense (COX; COX, 2000). 
Lastly, to recover coordinates from $B$, we first look at its rank. Since $B=X X^{T}$, we have

$$
\operatorname{rank}(B)=\operatorname{rank}\left(X X^{T}\right)=\operatorname{rank}(X)=p
$$

Hence, $B$ is of rank $p$ while also being symmetric and positive semi-definite. A well-known result from linear algebra is that it also has $p$ non-negative eigenvalues and $n-p$ zero eigenvalues (COX; COX, 2000). Writing $B$ in terms of its spectral decomposition,

$$
B=U \Lambda U^{T}
$$

where $\Lambda=\operatorname{diag}\left(\lambda_{1}, \lambda_{2}, \ldots, \lambda_{n}\right)$, with $\lambda_{1} \geq \lambda_{2} \geq \ldots \geq \lambda_{n}$, is the diagonal matrix of eigenvalues of $B$ and $U=\left[\begin{array}{llll}\mathbf{u}_{1} & \mathbf{u}_{2} & \cdots & \mathbf{u}_{n}\end{array}\right]$ is the matrix of eigenvectors with $\mathbf{u}_{i}^{T} \mathbf{u}_{i}=1$.

Because $B$ has $n-p$ zero eigenvalues and as $B=X X^{T}$ we write the coordinate matrix $X$ as

$$
X=U_{p} \Lambda_{p}^{\frac{1}{2}}
$$

where $U_{p}=\left[\begin{array}{llll}\mathbf{u}_{1} & \mathbf{u}_{2} & \cdots & \mathbf{u}_{p}\end{array}\right]$ and $\Lambda_{p}^{\frac{1}{2}}=\operatorname{diag}\left(\sqrt{\lambda_{1}}, \sqrt{\lambda_{2}}, \ldots, \sqrt{\lambda_{p}}\right)$. Note that since the eigenvectors have arbitrary signs, the solution is invariant regarding reflection in the origin.

One interesting aspect of classical scaling is that even when the given input distances are not Euclidean it is still possible to obtain a valid embedding in an Euclidean space if a condition is met. If $B$ is positive semi-definite and of rank $r$, then a configuration in a $r$-dimensional Euclidean space can be found (COX; COX, 2000). There are even other ways of satisfying this condition when the dissimilarities do not, as described by Cox e Cox (2000).

When considering some practical aspects, however, classical scaling has some limitations. Since the method relies on the spectral decomposition of a matrix whose number of elements grows quadratically on the dataset size, its applicability on larger datasets is hampered. To address that limitation, Landmark MDS (SILVA; TENENBAUM, 2004) was proposed.

Instead of performing the spectral decomposition on $B$ as explained above, a subset of the original input, called landmark points is selected. The selection can be done either randomly or by maximizing the distance between each landmark point. Then, classical scaling is applied on these points, resulting in an embedding $L$. Following, the embedding for the remaining points is found by performing a distance-based triangulation, with the embedding of instance $i$ given by

$$
\mathbf{x}_{i}=-\frac{1}{2} L^{\sharp}\left(\delta_{i}-\bar{\delta}\right)
$$

where $L^{\sharp}$ is the inverse transpose of $L, \delta_{i}$ is the vector of distances between $i$ and all landmark points and $\bar{\delta}$ denotes the vector of mean distances between all landmark points.

Even though it offers greater flexibility to describe other kinds of data and find embeddings which provide insight regarding their proximity, as given by their dissimilarities, classical scaling is not concerned with revealing what the analyst might consider as "interesting". Because of this, the following section discusses what has been done to provide a way for algorithms to 
find projections which describe interesting properties of the data, such as outliers and cluster formation.

\subsubsection{Projection Pursuit}

Finding "interesting" projections automatically while considering different properties of the data is, in a certain way, a generalization of the previous settings. Projection pursuit (PP) is the name coined by Friedman e Tukey (1974) to refer to techniques whose purpose is to find low-dimensional linear projections of multidimensional data by maximizing an objective function, called a projection index (HUBER, 1985). More formally, given a projection index $Q: \mathbb{R}^{q} \rightarrow \mathbb{R}$ and a dataset $X$, a PP finds a linear projection $A$ that satisfies

$$
A=\underset{P}{\arg \max }\{Q(X P)\}
$$

The index is defined with the intent of revealing some property in the data, such as outliers, holes or cluster formation. In particular, if $Q$ is chosen such that $A$ maximizes $\operatorname{tr}\left(A^{T} \operatorname{cov}(X) A\right)$ (problem (2.1)), then PP is equivalent to PCA. In fact, PCA has been pointed out as a special case of PP (HUBER, 1985). It should be noted that, even though it is possible to define mathematically what is an interesting projection, PP does not necessarily find an interesting projection, but merely increases the likelihood of that happening. Moreover, most indices rely on the hypothesis that normal projections should be deemed uninteresting (HUBER, 1985).

Despite being able to bypass the curse of dimensionality (HUBER, 1985), the high computational costs associated with PP has limited its applicability on general projection indices. Since it must assess many different projections in order to find the one that maximizes a certain index, while being prone to local optima, an interactive method where both the grand tour (section 2.4) and PP are used together has been proposed, called projection pursuit guided tour (COOK et al., 1995). It combines the exploration provided by a grand tour with the optimization criteria offered by PP indices. The user can manipulate individual attributes while the tour is running, further steering the optimization. By also letting the user decide when to activate the optimization index, the result is a highly interactive PP and grand tour.

Although offering a more compelling and objective analysis described by explicitly optimized functions, these are still linear methods. A direct implication of this fact is that they fail to find nonlinear relationships between attributes in the projections when those are present (MAATEN; POSTMA; HERIK, 2009). This limits their applicability in cases where the data being analyzed has such characteristics. As a result, nonlinear methods provide ways of circumventing this limitation by finding mappings that are capable of revealing these more complex relationships between attributes, although generally at the cost of having non-explicit mapping functions. 


\subsubsection{Sammon's Mapping}

One of the first nonlinear dimensionality reduction methods was proposed by Sammon (1969). Similarly to other dimensionality reduction scenarios, given a dataset $\mathscr{X}$ with dimensionality $p$, the objective is to transform $\mathscr{X}$ into a new dataset $\mathscr{Y}$ with dimensionality $q$ while approximately preserving the underlying structure of the data. In this case, however, it is assumed that the pairwise dissimilarities between each datum is what represents this structure.

As in the case of classical scaling (subsection 2.1.2), suppose we are given a set of dissimilarities between $n$ objects, with $\delta_{i j}$ representing the dissimilarity between objects $i$ and $j$. Let $d_{i j}=\left\|\mathbf{y}_{i}-\mathbf{y}_{j}\right\|$ for every $1 \leq i<j \leq n$. We say that a map $\mathscr{Y}$ preserves this structure if the error function given by

$$
E=\frac{1}{\sum_{i<j}^{n} \delta_{i j}} \sum_{i<j}^{n} \frac{\left(\delta_{i j}-d_{i j}\right)^{2}}{\delta_{i j}}
$$

is minimized. The proposed algorithm is based on a gradient steepest descent to minimize the error function in Equation (2.11). Starting with a random map, the coordinates of every $\mathbf{y}_{i}$ are adjusted for a fixed number of iterations $k$. Each iteration $t=1,2, \ldots, k$ updates the map using the equation

$$
\mathbf{y}_{i}^{(t+1)}=\mathbf{y}_{i}^{(t)}-\gamma \hat{\mathbf{y}}_{i}^{(t)}, \quad \hat{\mathbf{y}}_{i}^{(t)}=\left(\hat{y}_{i 1}^{(t)}, \hat{y}_{i 2}^{(t)}, \ldots, \hat{y}_{i q}^{(t)}\right)
$$

where

$$
\begin{gathered}
\hat{y}_{i k}^{(t)}=\frac{\partial E^{(t)}}{\partial y_{i k}^{(t)}} / \frac{\partial^{2} E^{(t)}}{\partial y_{i k}^{2(t)}} \\
\frac{\partial E^{(t)}}{\partial y_{i k}^{(t)}}=\frac{-2}{\sum_{i<j}^{n} \delta_{i j}} \sum_{j \neq i}^{n}\left[\frac{\delta_{i j}-d_{i j}}{\delta_{i j} d_{i j}}\right]\left(y_{i k}^{(t)}-y_{j k}^{(t)}\right), \\
\frac{\partial^{2} E^{(t)}}{\partial y_{i k}^{2(t)}}=\frac{-2}{\sum_{i<j}^{n} \delta_{i j}} \sum_{j \neq i}^{n} \frac{1}{\delta_{i j} d_{i j}}\left[\left(\delta_{i j}-d_{i j}\right)-\frac{\left(y_{i k}^{(t)}-y_{j k}^{(t)}\right)^{2}}{d_{i j}}\left(1+\frac{\delta_{i j}-d_{i j}}{d_{i j}}\right)\right] .
\end{gathered}
$$

Sammon (1969) suggests using $\gamma \approx 0.3$ or 0.4 as the "learning rate" in Equation (2.12).

Even though this method is well-known due to its simplicity, certain shortcomings restrict its applicability. First, it is computationally expensive, since each iteration requires $O\left(n^{2}\right)$ operations, amounting to a total of $O\left(\mathrm{kn}^{2}\right)$ operations. Additionally, the function being optimized is not convex, therefore the optimization is highly sensitive to the initial configuration, since it is crucial to avoiding local minima.

Pekalska et al. (1999) have proposed an approximation to address the high computational cost in the optimization. It is based on the hypothesis that if a linear mapping can approximate the map $\mathscr{Y}^{\prime}$ found by applying Sammon's method on a subset $\mathscr{X}^{\prime}$ of $c$ elements of $\mathscr{X}$, that same linear map can be used to also map the rest of the data. Formally, given the map $\mathscr{Y}^{\prime}$ and the matrix $D_{\mathscr{X}}$, whose entries represent the dissimilarities between each element of $\mathscr{X}^{\prime}$, we find a matrix $V$ that satisfies

$$
Y^{\prime}=D_{\mathscr{X}^{\prime}} V
$$


where $Y^{\prime}$ is the matrix form of $\mathscr{Y}^{\prime}$. Then, the map $\mathscr{Y}^{\prime \prime}$ of the remaining elements $\mathscr{X}^{\prime \prime}=\mathscr{X} \backslash \mathscr{X}^{\prime}$ is found by applying $V$ to $D^{\prime}$, with entries $\left[D^{\prime}\right]_{i j}=\delta\left(\mathbf{x}_{i}^{\prime \prime}, \mathbf{x}_{j}^{\prime}\right)$, where $\mathbf{x}_{i}^{\prime \prime} \in \mathscr{X}^{\prime \prime}$ and $\mathbf{x}_{j}^{\prime} \in \mathscr{X}^{\prime}$

$$
Y^{\prime \prime}=D^{\prime} V
$$

where $Y^{\prime \prime}$ is the matrix form of $\mathscr{Y}^{\prime \prime}$. Experiments conducted by Pekalska et al. (1999) have suggested that while this approach requires almost an order of magnitude less operations, the maps found are also close to those found by the original method.

Other approaches of mapping the dissimilarity between objects is based on modeling the points in the mapping as being connected by springs exerting a force proportional to their dissimilarity. These provide an alternative to Sammon's Mapping where either the iteration time or the number of iterations is reduced.

\subsubsection{Force-based point placement}

In this section, we present methods that map instances to points interacting with each other via forces proportional to their dissimilarities. Similarly to N-body simulations, these techniques place points on the mapping which represent each instance in the dataset and move them according to the forces exerted on them by the other points. The goal is to iteratively minimize, for each pair of instances $i$ and $j$, the difference between $\delta_{i j}$ and $d_{i j}$, where $\delta_{i j}$ is the dissimilarity between $\mathbf{x}_{i}$ and $\mathbf{x}_{j}$ and $d_{i j}=\left\|\mathbf{y}_{i}-\mathbf{y}_{j}\right\|$, resulting in a nonlinear mapping approach different from a direct optimization of an objective function.

Chalmers (1996) proposed a method of reducing the computational cost that involves calculating less interactions between points by making each point interact with a fixed number of other points. This is done by using two sets of points for each $\mathbf{y}_{i}$ : one representing the neighbors of $\mathbf{x}_{i}, \mathscr{V}_{i}$, and one representing other points selected at random, $\mathscr{S}_{i}$. They are both first initialized randomly, but only $\mathscr{V}_{i}$ gets carried over after each iteration, while $\mathscr{S}_{i}$ is not. The number of elements of each set is $\left|\mathscr{V}_{i}\right|=5$ and $\left|\mathscr{S}_{i}\right|=10$ for $i=1,2, \ldots, n$.

At every iteration, the position of each point $\mathbf{y}_{i}$ is updated by calculating the resulting force on it using elements in $\mathscr{V}_{i}$ and $\mathscr{S}_{i}$. Let $\mathbf{f}_{i j}$ be the force $\mathbf{y}_{j}$ exerts on $\mathbf{y}_{i}$, linearly proportional to $\delta_{i j}-d_{i j}$. The resulting force $\mathbf{f}_{i}$ applied on $\mathbf{y}_{i}$ is given by

$$
\mathbf{f}_{i}=\sum_{\mathbf{y}_{v} \in \mathscr{H}_{i}} \mathbf{f}_{i v}+\sum_{\mathbf{y}_{s} \in \mathscr{S}_{i}} \mathbf{f}_{i s}
$$

When every $\mathbf{f}_{i}$ is determined, an integration step using 4th order Runge-Kutta updates the position and velocity of each $\mathbf{y}_{i}$. In addition, other instances not already in $\mathscr{V}_{i}$ are selected to replace the ones in $\mathscr{S}_{i}$. Let $\delta_{i_{\max }}$ denote largest dissimilarity between some element in $\mathscr{V}_{i}$ and $\mathbf{x}_{i}$. For every new selected instance $\mathbf{x}_{j}$, if $\delta_{i j}<\delta_{i_{\max }}$, then the element with the largest dissimilarity is replaced by $\mathbf{x}_{j}$ in $\mathscr{V}_{i}$ and a new candidate is selected to be placed in $\mathscr{S}_{i}$, until $\mathscr{S}_{i}$ is again rebuilt. Therefore, over successive iterations, the set $\mathscr{V}_{i}$ tends to approximate better the real set of nearest neighbors. 
At the same time, $\mathscr{S}_{i}$ helps $\mathbf{y}_{i}$ avoid being stuck in local minima by adding random points to the exerted forces.

While the algorithm above certainly reduces the computational cost of calculating the exerting forces on each point, Morrison, Ross e Chalmers (2002) argue that it is not a substantial improvement, since the total number of operations is still roughly $O\left(n^{2}\right)$. Hence, they propose a new hybrid model where a subset $\mathscr{X}^{\prime}$ of $\sqrt{n}$ elements is mapped using Chalmers' spring model and the rest is interpolated.

This interpolation consists of mapping each new instance $\mathbf{x}_{i}$ close to the mapping $\mathbf{y}_{j}$ of $\mathbf{x}_{j} \in \mathscr{X}^{\prime}$ where $\delta_{i j}$ is minimal. Then, $S_{i}$ is constructed and used to calculate the resulting force on $\mathbf{y}_{i}$, refining its placement. When all instances are mapped, the full dataset goes through a fixed number of iterations of Chalmers' spring model to further improve the placement, in case the initial sampling was not representative enough to enable a faithful mapping. The algorithm results in $O(n \sqrt{n})$ operations, a significant improvement in running time over the previous method. Even though it has better running times, as shown in later studies, the algorithm can be stuck in local minima, failing to represent the original dataset faithfully (INGRAM; MUNZNER; OLANO, 2009, Figure 8).

Tejada, Minghim e Nonato (2003) proposes a modification to the N-body simulation where every point attracts or repels all others instead of computing the resulting force on each point and then moving the point. In other words, each iteration goes over all $\mathbf{y}_{i}$ and then updates the position of all $\mathbf{y}_{j}(j \neq i)$ by a fraction of the difference $\delta_{i j}-d_{i j}$. This approach leads to layout stabilization in only a few iterations, but as the number of operations on each iteration is $O\left(n^{2}\right)$, it is still costly.

As dealing with the raw number of operation in these scenarios has been a challenging subject, Ingram, Munzner e Olano (2009) proposed Glimmer, a technique that leverages the GPU for its raw processing power in order to reduce the time needed to perform the force calculations. By adapting a variation of Chalmer's spring model into an algorithm called GPU-SF, Glimmer employs a multi-level strategy that successively aggregates more subsets of the dataset into the layout which is then mapped using GPU-SF. While the authors argue that this multi-level strategy helps avoid local minima, there are no guarantees to that happening, as reported on later studies (FADEL et al., 2015, Figure 3).

Although these methods have dealt with the high computational costs of the explicit optimization of objective functions, the local optima problem is more challenging. Intuitively, convex objective functions and their guaranteed global optima should be the preferred approach. As discussed by Maaten, Postma e Herik (2009), though, this might not always be the case for dimensionality reduction problems. While the optimization of convex objective functions certainly finds global optima, they are more restricted in representing optimal solutions to the original problem, in this case a low dimensional map of multidimensional data. Consequently, their experiments suggest that finding suboptimal solutions to more reasonable non-convex 
objective functions is, in many cases, a more viable approach when compared to optimal solutions to convex objective functions.

Taking this into consideration, alternative approaches that also employ non-convex objective functions might still provide interesting results. Therefore, in the following section, we also present and discuss methods which take into account other properties of the data in their objective function.

\subsubsection{Stochastic Neighbor Embedding}

Instead of approximating the original distances in the mapping, the Stochastic Neighbor Embedding (SNE) (HINTON; ROWEIS, 2003) tries to represent the data by placing each mapped instance near a certain number of its original neighbors. This is done by defining probability distributions that represent the chance of each datum picking each other datum as its neighbor, with higher probabilities assigned to instances that are closer. Two distributions are calculated: one over the original data and another distribution over the current map. The map is then adjusted and its probabilities recalculated in order to approximate both distributions.

The probability distribution is calculated on the data by centering a Gaussian on each datum $\mathbf{x}_{i}$. We then compute the probability $p_{i j}$ that $\mathbf{x}_{i}$ would pick another datum $\mathbf{x}_{j}$ as its neighbor. Given a certain function $\delta$ that gives us the dissimilarity between $\mathbf{x}_{i}$ and $\mathbf{x}_{j}$, this probability is defined as

$$
p_{i j}=\frac{\exp \left(-\hat{\delta}_{i j}^{2}\right)}{\sum_{k \neq i} \exp \left(-\hat{\delta}_{i k}^{2}\right)}, \text { with } \hat{\delta}_{i j}^{2}=\frac{\delta\left(\mathbf{x}_{i}, \mathbf{x}_{j}\right)^{2}}{2 \sigma_{i}^{2}} .
$$

The value $\sigma_{i}$ determines how many neighbors affect the probabilities of each datum, effectively controlling the neighborhood size. As originally proposed by Hinton e Roweis (2003), it is calculated by a binary search that makes the entropy of the distribution over neighbors equal to $\log k$. The integer $k$, called perplexity, is set beforehand and is regarded as an upper bound on the neighborhood size determined by $\sigma_{i}$.

An analogous probability is calculated for the low-dimensional map, which we want to be such that it approximates the original data regarding the nearest neighbors of each instance. The induced probability $q_{i j}$ of $\mathbf{y}_{i}$ picking another $\mathbf{y}_{j}$ as its neighbor is given by

$$
q_{i j}=\frac{\exp \left(-\left\|\mathbf{y}_{i}-\mathbf{y}_{j}\right\|^{2}\right)}{\sum_{k \neq i} \exp \left(-\left\|\mathbf{y}_{i}-\mathbf{y}_{k}\right\|^{2}\right)} .
$$

Lastly, we minimize a cost function that measures how different the induced probabilities are from the original probabilities, with respect to the positioning of instances in the map. The cost function $C$, composed of a sum between all Kullback-Leibler divergences between the two probabilities for each object, is

$$
C=\sum_{i, j} p_{i j} \log \frac{p_{i j}}{q_{i j}}=\sum_{i} \operatorname{KL}\left(P_{i} \| Q_{i}\right) .
$$


Figure 2 - The information retrieval task in information visualization, as introduced by Venna et al. (2010). In this illustration, a query point $\mathbf{y}_{i}$ and its neighborhood $Q_{i}$ are evaluated against the original data, the instance $\mathbf{x}_{i}$ and its neighborhood $P_{i}$.
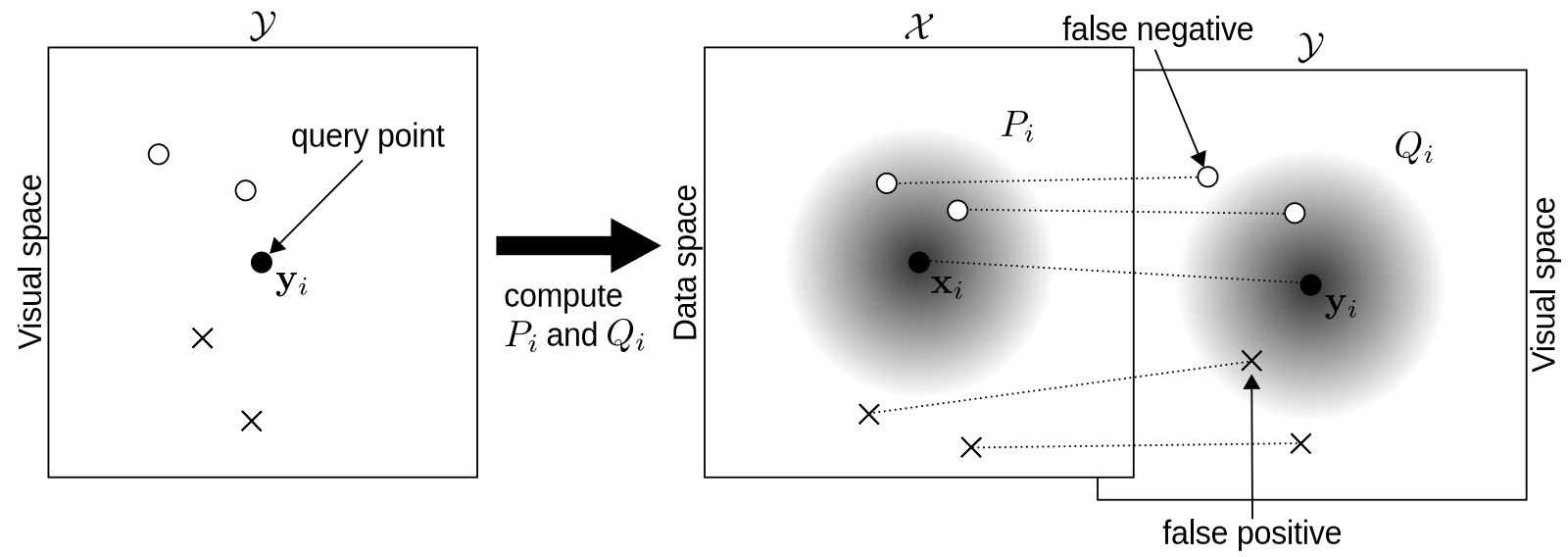

Source: Elaborated by the author.

This cost function has a desirable property of not only keeping close the points representing nearby instances, but also spreading apart points representing widely separated instances. For data where the number of dimensions is really high, such as visual and textual data, this property can be the source of the crowding problem (MAATEN; HINTON, 2008), where the large distances resulting from the sparsity of the data overshadow the smaller ones, leading to poor visual mappings. To overcome this, Maaten e Hinton (2008) propose the use of a t-distribution to define the probabilities $q_{i j}$ along with a simpler cost function. The justification for those modifications is that the t-distribution's characteristics help circumvent the crowding problem by treating clusters of distant elements in the same way as single points with small distances. Furthermore, the simpler cost function helps avoiding bad local optima.

Closely related to SNE is the Neighborhood Retrieval Visualizer (NeRV) technique, introduced by Venna et al. (2010). They describe visual inspection of visualizations as the task of retrieving information by selecting points (the query) from $\mathscr{Y}$ and inspecting its neighbors (the results of the query). Thus, the quality of $\mathscr{Y}$ can be evaluated as how good it is at "retrieving" the relevant neighbors in each query $\mathbf{y}_{i}$. Relevant neighbors, in this task, are the original neighbors of $\mathbf{x}_{i}$ in $\mathscr{X}$. These concepts are outlined in Figure 2.

By defining continuous neighborhoods as the probabilities of two points being neighbors, each neighborhood becomes the probability distributions defined earlier in Equation (2.19) and Equation (2.20). Then, they demonstrate that $\operatorname{KL}\left(P_{i} \| Q_{i}\right)$ is a generalization of recall, called smoothed recall, while $\mathrm{KL}\left(Q_{i} \| P_{i}\right)$ is a generalization of precision, called smoothed precision. The NeRV technique consists of optimizing a trade-off between these two measures. Since they cannot be both optimized, due the nature of their definition, the authors use a $\lambda$ parameter for controlling this trade-off, resulting in the cost function given by

$$
C_{\mathrm{NeRV}}=\lambda \sum_{i} \mathrm{KL}\left(P_{i} \| Q_{i}\right)+(1-\lambda) \sum_{i} \mathrm{KL}\left(Q_{i} \| P_{i}\right)
$$


From Equation (2.22), it follows that the SNE cost function is interpreted as maximizing the smoothed recall in the map, which is equivalent to setting $\lambda=1$.

\subsection{Interactive methods and techniques}

Methods discussed in the previous sections required little to no user intervention, meaning the user's ability to steer results was only parameter tuning. In this section, however, the user performs a central role in determining the resulting mapping. Since these techniques require a first step where a subsample of the data is selected to perform an initial mapping, the user is given the ability to directly manipulate this subsample. As discussed in the following sections, letting the user directly interfere on the initial mapping can lead to better mappings. By incorporating user knowledge indirectly on this first step, techniques which were not able to handle the complexity of certain datasets become able to do so.

\subsubsection{Least Squares Projection}

Among the first multidimensional projection techniques that allow user interaction, Least Squares Projection (LSP) (PAULOVICH et al., 2008) is a nonlinear technique that tries to map data by preserving neighborhood relationships. Similarly to Landmark MDS (SILVA; TENENBAUM, 2004), LSP employs a two-step approach to mapping the data.

First, elements of $\mathscr{X}$ are selected as representative samples. These samples should be chosen so as to represent the various groups of instances existing in $\mathscr{X}$. They are denoted by $\mathscr{X}^{\prime}=\left\{\mathbf{x}_{1}^{\prime}, \mathbf{x}_{2}^{\prime}, \ldots, \mathbf{x}_{c}^{\prime}\right\} \subset \mathscr{X}$. Their images $\mathscr{Y}^{\prime}=\left\{\mathbf{y}_{1}^{\prime}, \mathbf{y}_{2}^{\prime}, \ldots, \mathbf{y}_{c}^{\prime}\right\}$, called control points, are used to define the geometry of the final mapping, since neighboring points must be placed in similar locations in order to preserve the neighborhood structure in the data. Therefore, this step is critical regarding the visual quality of the mapping. The placement of control points can be done either by automatic techniques (section 2.1) such as those described in or by letting the user place them manually allowing analysts to directly interfere with the geometry of the mapping. A reasonable amount of elements must be chosen, both because there must be enough to properly represent the data and their number should not be too high as to exhibit prohibitive cost of interaction.

The second step is to map the remaining instances while attempting to place each of them near to its neighbors. Let $N_{i}=\left\{\mathbf{x}_{i_{1}}, \mathbf{x}_{i_{2}}, \ldots, \mathbf{x}_{i_{k}}\right\}$ be the set of neighbors of $\mathbf{x}_{i}$, where $k$ is a fixed constant. The coordinates of $\mathbf{y}_{i}$, the image of $\mathbf{x}_{i}$, is determined by the coordinates of the images of each neighbor of $\mathbf{x}_{i}$

$$
\mathbf{y}_{i}-\sum_{\mathbf{x}_{j} \in N_{i}} \alpha_{i j} \mathbf{y}_{j}=0, \quad \text { with } \alpha_{i j} \geq 0 \text { and } \sum \alpha_{i j}=1 .
$$

Equation (2.23) enforces the placement of $\mathbf{y}_{i}$ in the convex hull formed by the images of the neighbors of $\mathbf{x}_{i}$. The constants $\alpha_{i j}$ control the influence of each neighbor on the positioning 
of $\mathbf{y}_{i}$. In particular, for $\alpha_{i j}=\frac{1}{\left|N_{i}\right|}, \mathbf{y}_{i}$ is placed towards the centroid of the convex hull. Lower values of $\alpha_{i j}$ moves $\mathbf{y}_{i}$ away from neighbor $\mathbf{y}_{j}$, while larger values do the opposite. In order to map the remaining instances while trying to satisfy (2.23), a linear system of these equations is built. This problem is expressed in its matrix form as

$$
L Y=0
$$

where the solution $Y$ is the final mapping and $L_{n \times n}$ is given by

$$
[L]_{i j}= \begin{cases}1 & \text { if } i=j \\ -\alpha_{i j} & \text { if } \mathbf{x}_{j} \in N_{i} \\ 0 & \text { otherwise }\end{cases}
$$

An exact solution to (2.24), however, is not always possible, meaning that an approximate solution must be sought. One caveat is that the linear system (2.24) will always have a trivial solution: points are mapped to the same coordinates, which is undesirable. To avoid this, additional equations are added to account for the coordinates of control points, enforcing the solution to honor the previous placement of control points. This new problem can be expressed as

$$
A Y=\mathbf{b},
$$

where the solution $Y$ is the final mapping, $\mathbf{b}$ is given by

$$
b_{i}= \begin{cases}0 & \text { if } i \leq n, \\ y_{i} & \text { otherwise }\end{cases}
$$

and $A$ is $\left[{ }_{C}^{L}\right]$, with $C_{c \times n}$ given by

$$
[C]_{i j}= \begin{cases}1 & \text { if } \mathbf{y}_{j} \in \mathscr{Y}^{\prime} \\ 0 & \text { otherwise }\end{cases}
$$

The problem (2.25) is solved in a least squares sense, that is, minimizing $\|A Y-\mathbf{b}\|^{2}$, since an exact solution is unlikely to exist in most cases. In other words, we have $Y=\left(A^{T} A\right)^{-1} A^{T} \mathbf{b}$, which can be solved by an iterative method, such as conjugate gradient (PAULOVICH et al., 2008).

Experimental results presented by Paulovich et al. (2008) have shown that LSP has compelling performance in neighborhood preservation on the produced maps. A strong drawback, however, is the linear system employed: as it scales quadratically on the number of instances, solving it on larger datasets becomes computationally expensive. Other approaches that still enable user interaction while not solving large linear systems are more desirable in general.

\subsubsection{Part Linear Multidimensional Projection}

Based on a priori information given by already mapped points, such as the control points of LSP, the reasoning behind Part Linear Multidimensional Projection (PLMP) is that it is 
possible to determine a linear mapping that can approximate the transformation which positioned those points (PAULOVICH; SILVA; NONATO, 2010). Aside from being much faster than LSP, it also avoids the computation of distances between every datum, a mandatory operation in some techniques, such as those that rely on neighborhoods, which makes PLMP scalable to large numbers of instances.

A linear transformation $\Phi: \mathbb{R}^{p} \rightarrow \mathbb{R}^{q}$ that minimizes the difference between the projected distances $d_{i j}$ and the original dissimilarities $\delta_{i j}$ can be expressed as

$$
\Phi=\underset{\hat{\Phi} \in \mathscr{L} p, q}{\arg \min }\left\{\frac{1}{\sum_{i<j}^{n} \delta_{i j}^{2}} \sum_{i<j}^{n}\left(\delta_{i j}-\left\|\hat{\Phi}\left(\mathbf{x}_{i}\right)-\hat{\Phi}\left(\mathbf{x}_{j}\right)\right\|\right)^{2}\right\}
$$

where $\mathscr{L}_{p, q}$ is the space of linear transformations from $\mathbb{R}^{p}$ to $\mathbb{R}^{q}$. As pointed by (PAULOVICH; SILVA; NONATO, 2010), solving (2.26) directly is computationally expensive as $n$ grows. Consequently, an approximation of $\Phi$, denoted $\tilde{\Phi}$, is proposed by using the already given a priori information by the representative samples.

By assuming that the number of representative samples $\mathscr{X}^{\prime}=\left\{\mathbf{x}_{1}^{\prime}, \mathbf{x}_{2}^{\prime}, \ldots, \mathbf{x}_{c}^{\prime}\right\}$ is greater than $p, \Phi$ can be solved, again in the least squares sense, through $q$ linear systems in the form

$$
X^{\prime T} X^{\prime} \phi_{j}=X^{\prime T} \mathbf{b}_{j}
$$

for all $1 \leq j \leq q$, where $X^{\prime}$ is the matrix form of $\mathscr{X}^{\prime}$ and $\mathbf{b}_{j}$ is the $j$-th column of $Y^{\prime}$, which is the image of $X^{\prime}$. Then, each $\phi_{j}$ composes a column of $\tilde{\Phi}$. Lastly, all the remaining instances are projected using $\tilde{\Phi}$, the approximation of $\Phi$.

The experimental results by Paulovich, Silva e Nonato (2010) show that PLMP is indeed one of the fastest techniques when projecting to two dimensions. Moreover, it also attains good efficacy regarding the visual quality of projections. The authors also provide further evidence that user interactivity can indeed improve the resulting maps. Nonetheless, it is still a global transformation: all points undergo the same transformation, so changing what calculates the transformation (in this case, the initial positioning provided), also incurs in global changes on the final map. As a consequence, user interaction is hampered by the unintuitive possible changes in distant places on the map caused by changes in the control points. This issue is addressed by another technique, which creates local mapping functions for each control point, described in the next section.

\subsubsection{Local Affine Multidimensional Projection}

Mapping groups of data using local mapping functions was already a characteristic of other techniques, such as the ones proposed by Morrison, Ross e Chalmers (2002), Chalmers (1996), Paulovich et al. (2011a). However, one of their main drawbacks is using neighborhood graphs to define groups of data, hence arising the notion of "locality". The construction of such graphs usually relies on other parameters such as a maximum distance to consider one datum as 
a neighbor of another, which must be tuned for each dataset for optimal operation. The Local Affine Multidimensional Projection (LAMP) technique has properties that overcome previously mentioned shortcomings (JOIA et al., 2011).

The solution adopted by LAMP to locally mapping each instance $\mathbf{x}_{i}$ is to find one linear function for each of them. The problem is defined in terms of the already known coordinates of each representative sample and the mapping of each $\mathbf{x}_{i}$ is the affine transformation $f_{\mathbf{x}_{i}}(\mathbf{x})=\mathbf{x} M+\mathbf{t}$ that optimizes

$$
\begin{array}{ll}
\underset{f_{\mathbf{x}_{i}}}{\operatorname{minimize}} & \sum_{j=1}^{c} \alpha_{j}\left\|f_{\mathbf{x}_{i}}\left(\mathbf{x}_{j}^{\prime}\right)-\mathbf{y}_{j}^{\prime}\right\|^{2} \\
\text { subject to } & M^{T} M=I,
\end{array}
$$

where $\alpha_{j}=\frac{1}{\left\|\mathbf{x}_{j}^{\prime}-\mathbf{x}_{i}\right\|^{2}}$ are scalar weights added in order to let more similar representative samples influence more the mapping of $\mathbf{x}_{i}$, while, conversely, less similar ones have less influence. Furthermore, removing altogether the influence of more distant representative samples is what enables true local mappings.

The constraint added to the problem (2.28) enforces a transformation that only rotates and translates the data. This has a desirable property that no distortion is added from scale or shear effects, helping minimize the differences between the original dissimilarities and the distances in the map. Additionally, the distortion (regarding dissimilarities) added by the positioning of representative samples is mitigated. With no constraints, a solution could be found by using a traditional least squares fitting, as seen in other techniques, but would severely propagate the distortions (JOIA et al., 2011).

The solution to the minimization problem (2.28), is to rewrite it in matrix form, giving rise to an Orthogonal Procrustes Problem (GOWER; DIJKSTERHUIS, 2004), which has known solution. To do this, we take the partial derivatives with respect to $\mathbf{t}$ equal to zero and rewrite $\mathbf{t}$ in terms of $M$ as $\mathbf{t}=\overline{\mathbf{y}}^{\prime}-\overline{\mathbf{x}}^{\prime} M$, where

$$
\overline{\mathbf{x}}^{\prime}=\frac{\sum_{j=1}^{c} \alpha_{j} \mathbf{x}_{j}^{\prime}}{\sum_{j=1}^{c} \alpha_{j}}, \quad \overline{\mathbf{y}}^{\prime}=\frac{\sum_{j=1}^{c} \alpha_{j} \mathbf{y}_{j}^{\prime}}{\sum_{j=1}^{c} \alpha_{j}} .
$$

Back to problem (2.28), we have

$$
\begin{array}{ll}
\underset{f_{\mathbf{x}_{i}}}{\operatorname{minimize}} & \sum_{j=1}^{c} \alpha_{j}\left\|f_{\mathbf{x}_{i}}\left(\hat{\mathbf{x}}_{j}\right)-\hat{\mathbf{y}}_{j}\right\|^{2} \\
\text { subject to } & M^{T} M=I,
\end{array}
$$

where $\hat{\mathbf{x}}_{j}=\mathbf{x}_{j}^{\prime}-\overline{\mathbf{x}}^{\prime}$ and $\hat{\mathbf{y}}_{j}=\mathbf{y}_{j}^{\prime}-\overline{\mathbf{y}}^{\prime}$. Lastly, by writing problem (2.29) in matrix form, we have

$$
\begin{array}{ll}
\text { minimize } & \|A M-B\|_{F} \\
\text { subject to } & M^{T} M=I,
\end{array}
$$


where $\|\cdot\|_{F}$ denotes the Frobenius norm and matrices A and B are given by

$$
A=\left[\begin{array}{c}
\sqrt{\alpha_{1}} \hat{\mathbf{x}}_{1}^{T} \\
\sqrt{\alpha_{2}} \hat{\mathbf{x}}_{2}^{T} \\
\vdots \\
\sqrt{\alpha_{c}} \hat{\mathbf{x}}_{c}^{T}
\end{array}\right], \quad B=\left[\begin{array}{c}
\sqrt{\alpha_{1}} \hat{\mathbf{y}}_{1}^{T} \\
\sqrt{\alpha_{2}} \hat{\mathbf{y}}_{2}^{T} \\
\vdots \\
\sqrt{\alpha_{c}} \hat{\mathbf{y}}_{c}^{T}
\end{array}\right] .
$$

As mentioned before, problem (2.30) has well-known solution. By writing

$$
A^{T} B=U D V^{T}
$$

where $U D V^{T}$ is the singular value decomposition (SVD) of $A^{T} B$, we have that $M=U V^{T}$. We now have all we need to calculate $f_{\mathbf{x}_{i}}$, which gives us $\mathbf{y}_{i}$,

$$
f_{\mathbf{x}_{i}}(\mathbf{x})=\left(\mathbf{x}-\overline{\mathbf{x}}^{\prime}\right) M+\overline{\mathbf{y}}^{\prime}
$$

In addition to having a function for each instance being mapped, LAMP can also have an even more local behavior by using only the closest control points to the instance being mapped. With this approach, the amount of disturbance a control point can cause in a map is more limited, creating discontinuities that can lead to more predictable (and intuitive) mapping behavior while interacting.

The introduction of local affine mappings for each data point, while providing a mathematical framework that is both efficient and efficacious, places LAMP among the state-of-the-art multidimensional projection techniques. These traits are central to user interactivity, since fast response times and mappings that allow local modifications enable a new range of applications (JOIA et al., 2011). Nevertheless, the initial mapping has no restrictions on positioning by the user. Additionally, there are no studies regarding the distortion introduced by the manipulation, either in terms of the visual quality or the plausibility of the resulting maps. As a consequence, when control points no longer reflect the original data, little can be inferred from the final mapping attained.

\subsection{Interactive data transformation}

User interactivity in projections is not limited to changing the initial placement of a subsample to improve the positioning of the mapping. Two other applications are presented in this section: interactive distance metric learning and interactive data transformation.

\subsubsection{Dis-function}

In distance metric learning, one is interested in finding a distance metric that can appropriately measure the similarity of objects based on certain constraints. As an example, content-based 
Figure 3 - An example of interaction in Dis-function. (a) The blue and red sets are defined and the points are moved in accordance to their class coloring. (b) The updated view with a new distance metric reflects the previous manipulation.

(a)

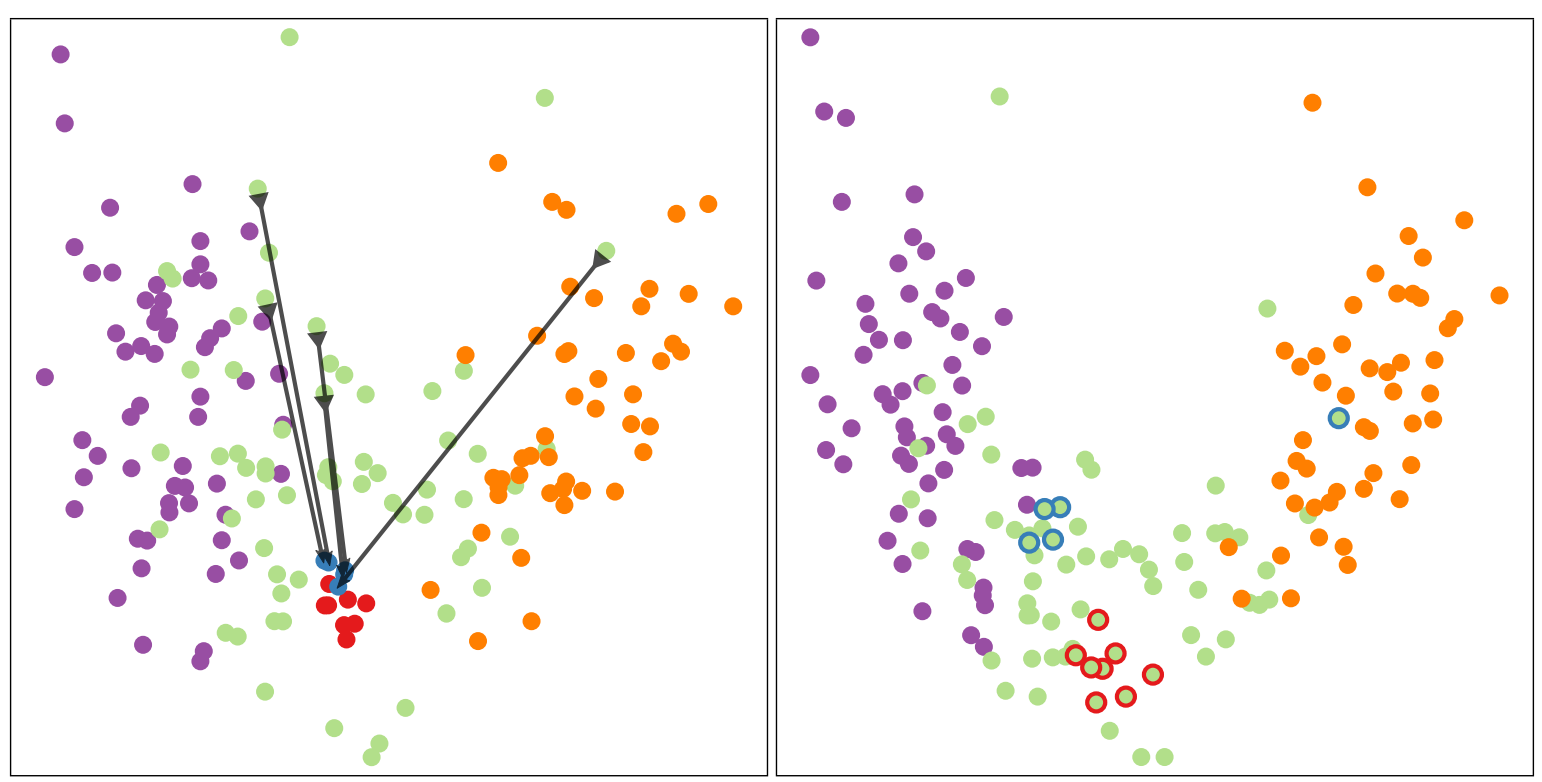

Source: Brown et al. (2012).

(b)

$$
\text { . }
$$

image retrieval (CBIR) systems rely mostly on the distance metric in use to determine the similarity between images. Consequently, the quality of a query is highly sensitive to the distance metric used. Usually, the constraints presented to the distance metric learning method are class labels where members of the same class are assumed to be similar and members of distinct classes are assumed to be dissimilar. Then, learning algorithms are used to find a metric that can satisfy those constraints, effectively obtaining a distance metric from data (LIU, 2006).

In this context, Dis-function (BROWN et al., 2012) is a distance metric learning system where the constraints are presented to the method via an interactive visualization of the dataset. The distance metric being learned in this case is defined by

$$
\delta_{\Theta}\left(\mathbf{x}_{i}, \mathbf{x}_{j}\right)=\sum_{k=1}^{p} \theta_{k}\left(x_{i k}-x_{j k}\right)^{2}
$$

where $\Theta=\left(\theta_{1}, \theta_{2}, \ldots, \theta_{p}\right)$ is the vector of coefficients being learned, initialized to $\theta_{k}=\frac{1}{p}$, for $k=1,2, \ldots, p$. By presenting a scatterplot of the data, projected using classical scaling, the system expects the user to compose two sets of points, one blue and one red, which will have their distances updated. After selecting which points will compose both sets, the user proceeds to dragging the points. When the user is satisfied with the new positioning, a button press confirms the update and $\Theta$ is adjusted automatically to reflect those changes. An example of such changes are illustrated in Figure 3. As the projection uses the same distance metric $\delta_{\Theta}$, the view is updated with the new metric and the user can then proceed to further refinement of $\Theta$.

The novel ideas of combining interactivity with machine learning brought by Dis-function 
provide a step ahead towards incorporating user knowledge both in data analysis and information retrieval systems. An important aspect is that the user is capable of immediately seeing the changes caused by interaction. This provides intuitive knowledge about the inner workings of the learning system while allowing the user to better decide which step to take next when interacting with it. As a consequence, since the user will be more familiar with the distance metric learned, he or she will be able to better judge where the new metric will be applicable, if at all.

Those benefits do not come without its shortcomings, however. As the system employs classical scaling to reflect the changes to the metric being learned, it is limited to a small number of instance being analyzed. While the learning system is much faster than the MDS algorithm, in the case of roughly 2,000 instances, the user must wait more than 2,000 seconds (BROWN et al., 2012) before the view is updated, which severely hampers interactivity.

\subsubsection{User-driven feature space transformation}

Another application of user interaction in projections was proposed by Mamani et al. (2013). Motivated by the lack of an overview of the data while the user performs transformations on the data attributes on existing techniques, a new system where the user is able to manipulate a sample of the data and then view the changes incurred on the entire dataset is presented.

The user is able to transform the dataset indirectly by changing the mapping $\mathscr{Y}^{\prime}$ of a subsample $\mathscr{X}^{\prime}$ using straightforward dragging operations. Then, from the new distances $d_{i j}^{\prime}$ for every $\mathbf{y}_{i}, \mathbf{y}_{j} \in \mathscr{Y}^{\prime}$, a new dissimilarity $\delta_{i j}^{\prime}$ is estimated using $\delta_{i j}$, the original $d_{i j}$ and $d_{i j}^{\prime}$. In order to reflect this transformation back on the data, Force Scheme (described in subsection 2.1.5) was adapted to take place in $\mathbb{R}^{p}$. Then, after the samples are updated in $\mathbb{R}^{p}$, LAMP (described in subsection 2.2.3), also using a modified formulation to transform the data in $\mathbb{R}^{p}$, is used to update rest of the data, reflecting the user transformation.

The decision to use LAMP on those transformations was crucial in two aspects. First, its orthogonality constraints prevents successive cycles of transformations from collapsing or expanding mapped points much beyond what the user intended. Second, its notorious ability to obtain satisfactory results even when the number of subsamples used is small, which is decisive in reducing visual clutter and user effort during manipulation.

Mamani et al. (2013) also presented an image retrieval system where the user can, using the proposed transformation framework, implicitly define new clustering criteria using intuitive dragging operations. As shown in Figure 4, by dragging apart some sample images, a transformation on data attributes incurs a better retrieval of similar images.

Even though the proposed framework provides new interesting applications, it its not complete. When considering the transformation caused by the subsample manipulation, it is not clear how far the user can go before the transformation disrupts the original data to a point where it no longer has any meaning. In addition, even when the transformation is apparently coherent 
Figure 4 - The image retrieval system prototype by Mamani et al. (2013). The user drags a collection of images and attempts to group them using a subjective grouping criteria from the given subsample of the data. Then, the returned images after transformation fit better the criteria implicitly given by the user through the subsample manipulation.

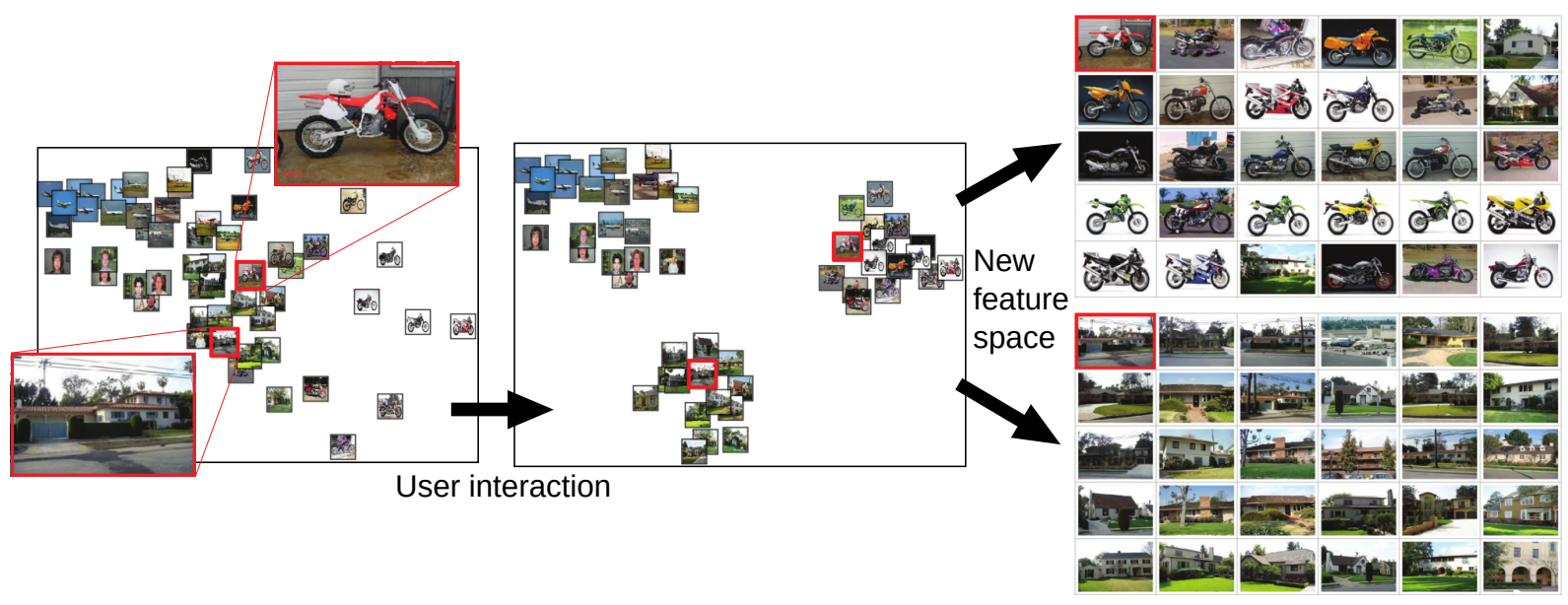

Source: Mamani et al. (2013).

from the user's point of view, the loss of information in the sample projection can introduce a bias in two ways. First, by leading the user to think certain malformed groups in the mapping are also malformed in the original data, the information loss in the mapping is then propagated back through the transformations, while the user is unaware of such situation. Second, since the user cannot assess how faithful the current transformed data is to the original data, the results attained cannot be used to perform further analysis.

\subsection{Animation and transition-based projections}

While previous techniques use interaction as a means of incorporating user knowlodge in some form, the ones presented in this section use interaction to enable visual data exploration (KEIM, 2002). Specifically, the purpose of visual data exploration is to integrate the human in the data exploration process, leveraging its perceptual abilities to the analysis of large data sets. It is particularly useful when either the goals of exploration are not clearly defined or little is known beforehand about the data.

\subsubsection{Grand Tour}

When exploring multidimensional data via linear projections, the simplest approach would be to observe different projections with the intent of "looking" at the data from different perspectives. However, choosing which perspectives to look at can be a daunting task at first. The grand tour (ASIMOV, 1985) is a method devised for visual exploration of multivariate data via orthogonal projections onto a sequence of two-dimensional subspaces which addresses this issue. 
Figure 5 - Illustration of a three-dimensional grand tour path containing three projection planes: $Y_{1}, Y_{2}$ and $Y_{3}$. The tour consists of an animation traversing a path of projections that are interpolated between certain projection planes that represent the properties described by Asimov (1985) using a smooth shortest path rotation.

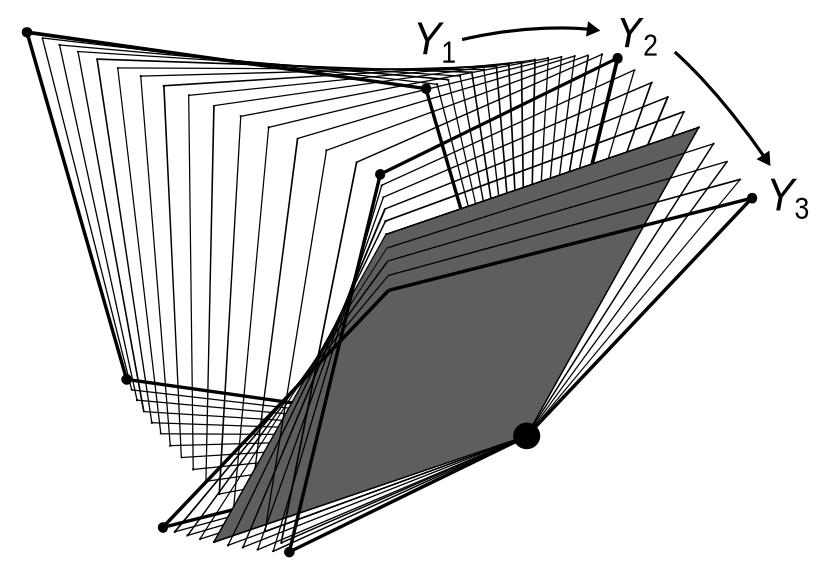

Source: Theus (2008).

These projections are selected so that the sequence is dense, that is, it should be asymptotically close to the sequence of all two-dimensional projections. This restriction accomplishes a desirable property that the user will see the data through all relevant perspectives, disregarding rotation and scaling, without having to go through the sequence of all two-dimensional projections. It should be noted that this alone does not make the sequence of projections finite. Therefore, it is best to set the number of projections $t$ in advance, not only making the task feasible, but also allowing for better optimization of the properties of the projection sequence (ASIMOV, 1985).

Three different methods for determining the sequence of projections are proposed by Asimov (1985). This sequence of projections, $Y_{1}, Y_{2}, \ldots, Y_{t}$, compose the path to be traversed in the analysis. A grand tour is accomplished by traversing this path using animated transitions between each $Y_{i}$ and $Y_{i+1}$ via a shortest path rotation between the two. Figure 5 illustrates a tour over a three-dimensional space, highlighting the current plane being displayed and $Y_{1}, Y_{2}$ and $Y_{3}$, which represent hypothetical projections satisfying the density criteria.

Although rotations incur an analogy to physical 3D rotations, subjectively increasing its aesthetic appeal, in higher dimensional datasets, however, they become less intuitive for an observer expecting the same behavior, hindering the analysis. Another serious drawback is that number of projections that the user must sit through with a higher number of dimensions makes for an infeasible task given the amount of time that must be spent (HUBER, 1985).

Extensions of the method were proposed by Cook et al. (1995), adding more interactivity. Selecting an attribute, the user can control the influence that attribute has on the projection by applying "drag" operations on the visualization. This drag operation is analogous to the 3D manipulation of objects, where the surface is dragged in order to apply a rotation. A direct consequence of this added interactivity is that, whenever an interesting or close to interesting projection is seen, the animation can be interrupted and the user can then apply slight modifications 
Figure 6 - The transition between two scatterplots in the scatterplot matrix navigation framework proposed by Elmqvist, Dragicevic e Fekete (2008). Each step of the transition is indicated by the labels (a) through (e).

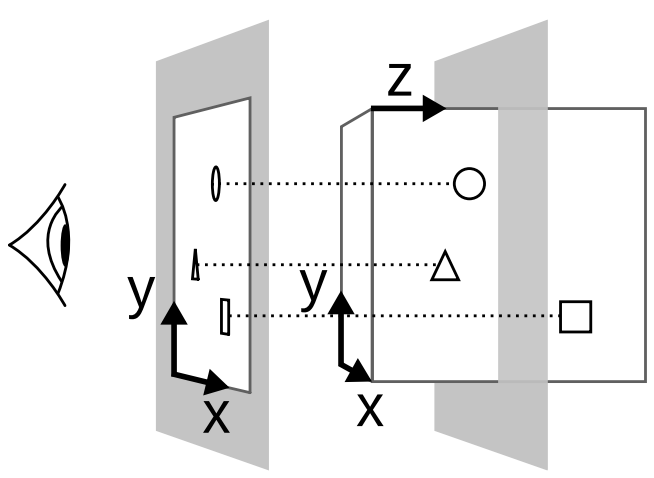

(a)

(b)

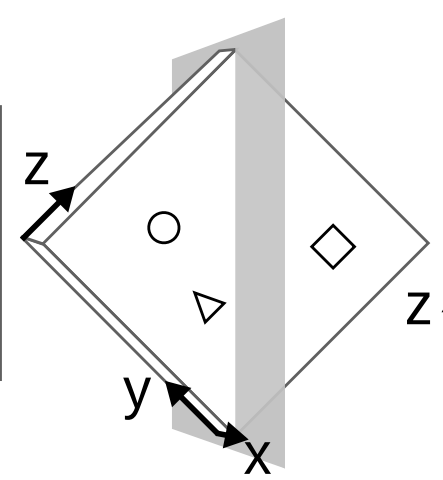

(c)

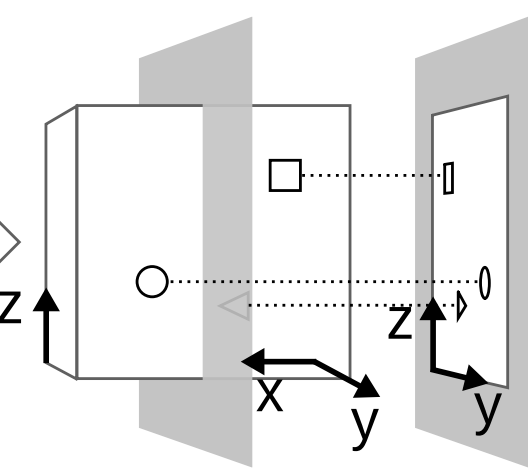

(d)

(e)

Source: Elmqvist, Dragicevic e Fekete (2008).

to assist exploration. Adding interactivity to the grand tour, however, does not circumvent the problem of analysing a long animation of projections when the number of attributes is large. As the objective of multidimensional data exploration is finding interesting projections that expose properties of the data, being able to explicitly choose which property to expose is a desirable feature.

\subsubsection{Scatterplot matrix navigation}

Inspired by the ideas of finding interesting projections from the grand tour (subsection 2.4.1) and projection pursuit (subsection 2.1.3), Elmqvist, Dragicevic e Fekete (2008) proposed a method of visual exploration where the user decides which projections to see next. The rationale behind it is that automatic methods provide no guarantee that the projections found will be interesting as judged by the user, since their notion of "interest" is very specific and limited.

The proposed exploration method is based on the navigation of a collection of scatterplots. A scatterplot for each pairwise combination of attributes from the dataset is built and organized into a scatterplot matrix. This matrix has two main purposes: providing an overview of the dataset and clues to interesting projections. Once selected, the scatterplot on a cell is shown at a larger view.

For transitioning between two different scatterplots, they must have one attribute in common. This helps the user to keep context while exploring different views of the data. Preserving one of the attributes, the view is first extruded with the new attribute acting as a depth coordinate (effectively making the scatterplot three-dimensional) and then rotated in order to make the replaced attribute the new depth coordinate, which is squashed into the plane, making the scatterplot two-dimensional again. This is illustrated in Figure 6. 
Figure 7 - The different ways of cell navigation in the scatterplot matrix navigation framework proposed by Elmqvist, Dragicevic e Fekete (2008).
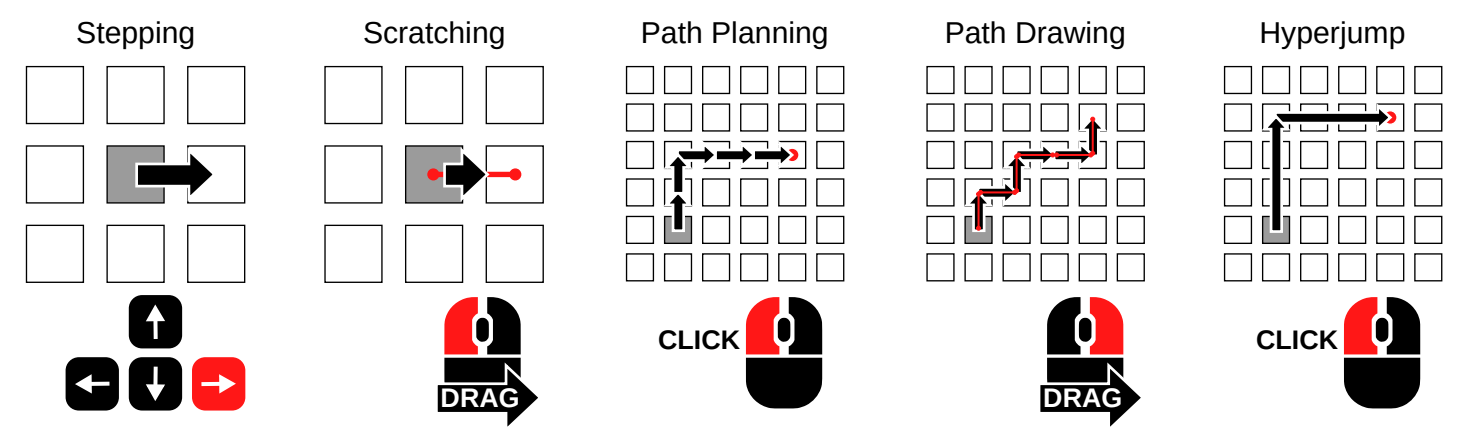

Source: Elmqvist, Dragicevic e Fekete (2008).

The navigation between cells offers several options, illustrated by Figure 7. The simplest is stepping, which allows transitions between adjacent cells using the arrow keys on a keyboard. Scratching relies on mouse drags. By dragging with the left mouse button between cells, the user controls the transition speed and can go either forward or backwards. In path planning, the user simply clicks a target cell and the system finds a shortest path to the cell, displaying all transitions in between. Path drawing instead leaves the path creation to the user: by dragging the cursor with the right mouse button through cells, the system shows all transitions between the cells in that path. Lastly, hyperjump allows a fast transitioning to any cell by performing at most two transitions.

Its startling simplicity and ease of use makes an attractive data exploration method for casual users of data visualization. Notwithstanding, the generation of a scatterplot matrix with all possible selections of two attributes imposes limitations on datasets of high dimensionality. Since the number of cells in the matrix is $p^{2}$, the visual clutter from the scatterplot matrix alone imposes severe limitations on visual exploration.

\subsection{Chapter conclusions}

We discussed and presented methods related to multivariate data visualization and analysis using projections. As previously stated, these methods aim at or were later repurposed for representing data as glyphs in a scatterplot, summarizing their numerous dimensions into two or three dimensions. In most cases, however, this comes at the cost of exhibiting only a limited set of patterns in the data.

Automatic methods require no user input beyond the data under analysis. As a consequence, the very nature of the method is going to be the only factor in determining the results. Whenever the results are poor, the user must find another method adequate for the data. This means that beyond parameters, whenever the results are insatisfactory to draw conclusions from, the user has no other option. In addition, their "black box" nature keeps the user from 
understanding what went wrong in some cases.

Alternatively, interactive methods provide the user a mechanism of directly steering the results. Because of this, the user performs a key role in the results attained: not only being part of the process, users can also intervene with their additional knowledge, incorporating it indirectly through interaction. As presented in the previous sections, this additional knowledge can lead to better results, overcoming the limitations of the methods in use when they cannot handle the complexity of the data being analyzed. Another positive aspect of interactivity in visualization comes from directly seeing the results of the interaction, including better understanding of the underlying algorithm.

Nevertheless, the state of the art methods mentioned in this chapter have some limitations in their interaction mechanisms. The most prominent limitation is related to the freedom given to users: as they manipulate the points, no restriction is given whatsoever to the movement. Thus, the manipulation does not necessarily reflect the data and can lead to distorted results. Moreover, the final mappings attained cannot be assessed regarding this distortion, which incurs limitations on the analysis conducted afterwards. 



\section{VISUALLY UNDERSTANDING MANIPULATION}

Multidimensional projection techniques help us visualize and understand complex high dimensional data in an intuitive way using lower dimensional representations of such data. Interactive multidimensional projection techniques are a special class of such techniques that introduce new ways to analyze and perceive data via interactive repositioning of elements. Interactions with such maps are largely driven by intuitive repositioning of points. As a result, it can often lead to a trial and error process.

One reason for this fact is that these interactions happen in a representation whose dimensionality is much lower than the dimensionality of the data. This mismatch sometimes results in counter-intuitive effects during manipulations. Another reason is the use of control points to perform such repositioning (section 2.2). Control points are a subset of the original data, usually chosen as representative of the whole dataset, and the position of every other data item, which we refer to as regular points, is computed based on the position of each control point. Thus, whenever repositioning a small set of regular points, we must identify the correct control points for the task, but it is not always clear which control points are the most relevant in positioning those points.

In this chapter, we discuss the design and implementation of a collection of data visualization techniques employed in a concise context for addressing these difficulties. Specifically, we have two aims with these visualizations. First, providing the information necessary to choose the control points with the best potential to perform the desired manipulation when interacting. Second, highlighting the changes caused by such manipulation. Note that we consider the control points as being already defined. In other words, the user, when interacting with a projection, does not have the option to determine which points are going to be control points or not. While it could lead to interesting exploration scenarios, we consider this kind of interaction out of the scope of our visualizations techniques. 
Throughout the chapter, we present our visualizations and design choices using examples with the LAMP technique (subsection 2.2.3) and the SEGMENTATION dataset. This dataset (see Table 1 for more details) was chosen for these examples due to the number of instances and dimensions being adequate for illustrating our ideas: not too large to become a challenging visualization problem and causing visual clutter, while not too small to be only a toy example with little meaning in a more complex scenario. In our examples, we randomly selected 137 $(3 \sqrt{n})$ instances to become control points. Their initial positions were computed using the Force Scheme technique (subsection 2.1.5).

\subsection{Selecting control points}

When interacting with a multidimensional projection that allows manipulation, the first step is to determine which control points are going to be repositioned. This selection ${ }^{1}$ is essential to the manipulation because it determines which instances are going to be affected and repositioned, having considerable influence on the final layout. The decision of moving certain control points is also based on the employed technique, not only on the current layout. Consequently, it must be based on more information than is trivially available to the user.

\subsubsection{Regions of interest}

Since control points determine which regions are going to be affected, we must first identify which regions might be in our interest to modify. In order to do this, a measure of what is in our interest to change in a map is necessary, so that we can in turn determine which control points would be the most adequate to reposition. Since this measure is dependent on the application domain, we do not impose a specific measure here. Instead, we illustrate what is interesting in a somewhat general sense by adopting a per-point measure, called aggregated error. As the name suggests, it measures the accumulation of errors: either by positioning a point next to false neighbors or away from its true neighbors. These errors, called projection errors, between two points $i$ and $j$ are given by

$$
e_{i j}=\frac{\delta\left(\mathbf{x}_{i}, \mathbf{x}_{j}\right)}{\max _{i, j}\left\{\boldsymbol{\delta}\left(\mathbf{x}_{i}, \mathbf{x}_{j}\right)\right\}}-\frac{\left\|\mathbf{y}_{i}-\mathbf{y}_{j}\right\|}{\max _{i, j}\left\{\left\|\mathbf{y}_{i}-\mathbf{y}_{j}\right\|\right\}} .
$$

Negative errors indicate that $i$ is closer than it should be to $j$, while positive errors indicate the opposite. The aggregated error on a point $i$ is the sum of the absolute errors, given by

$$
e_{i}^{\text {aggr }}=\sum_{j \neq i}\left|e_{i j}\right|
$$

Note that, while the projection error is bounded within $[-1,1]$ due to normalization factors, the aggregated error is not.

1 Here, "selection" has the conventional user interface meaning of informing in some way that the user is interested in performing some operation with a certain visual element, in this case, a control point. 
Figure 8 - Highlighting regions of interest with the technique described by Martins et al. (2014) applied only to regular points (non control points). Control points are the larger, white circles.

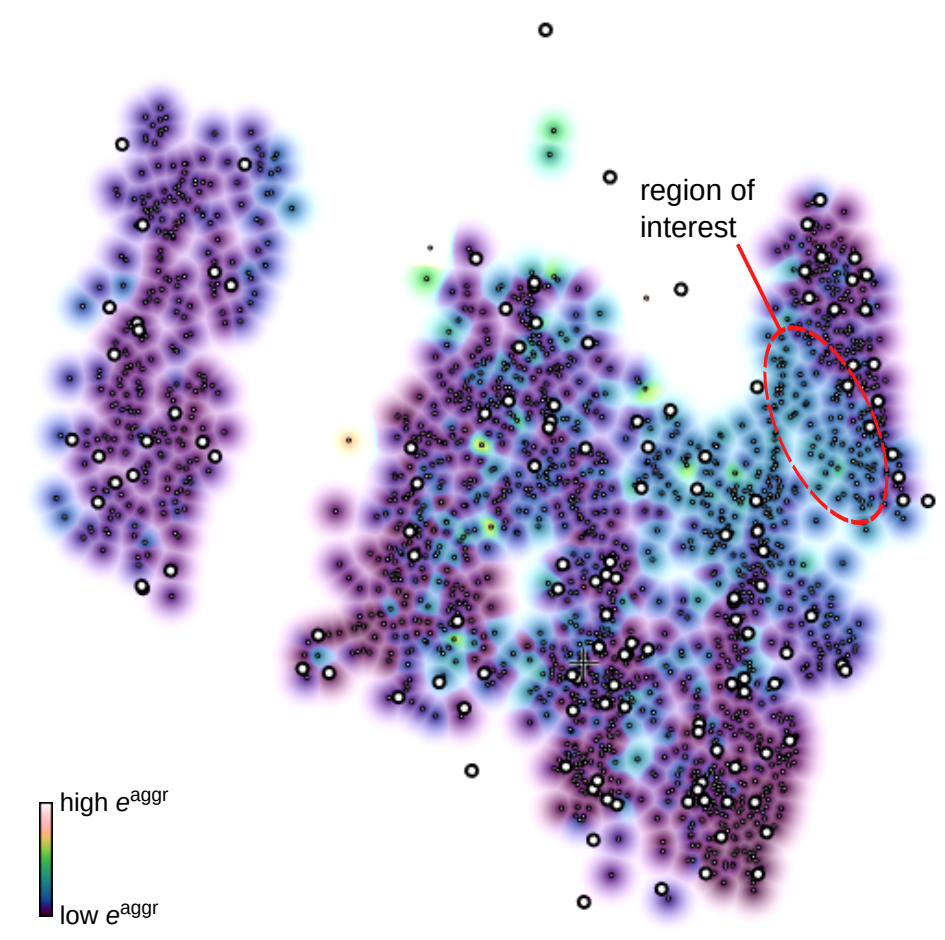

Source: Elaborated by the author.

With a way to determine interest in our projection, we need to highlight regions that have higher values of our interest measure. Some solutions for this include CheckViz (LESPINATS; AUPETIT, 2011) and the collection of methods by Stahnke et al. (2016). On one hand, the methods by Stahnke et al. (2016) are tailored for correcting and examining errors in projections defined in a specific sense. We are more concerned with representing any measure visually so that we can highlight potential regions of interest for interaction. CheckViz, on the other hand, is much simpler and can be adapted to our scenario. Despite this, the use of Voronoi diagrams has the drawback of attracting attention to outlier points in the map, since their cell will be larger than others. Thus, we opted for the rendering technique used by Martins et al. (2014) applied to visual analysis of dimensionality reduction. The major advantage of such technique is the similar flexibility of the visual representation proposed by Lespinats e Aupetit (2011), but with limited cell size around points. With this, we provide simple highlighting to regions of the map with large errors without hampering the analysis with points that should not be drawing attention.

To illustrate our methodology, suppose that we are interested in interacting with a certain projection of the SEGMENTATION dataset and our intent to segregate a cluster of instances using the projection error as a guidance for our interaction. That is, suppose we are testing the hypothesis of whether a certain group of points could be placed further away than it currently is, while not making a bad compromise in terms of the projection error. For this, we would first identify and then select a region using the splat visualization as a guide, as illustrated in Figure 8. 
Figure 9 - Selection of control points using influence as guide. The selection of control points changes the values mapped by the splat from $e^{\text {aggr }}$ to influence (accumulated $\alpha_{i j}$ 's).

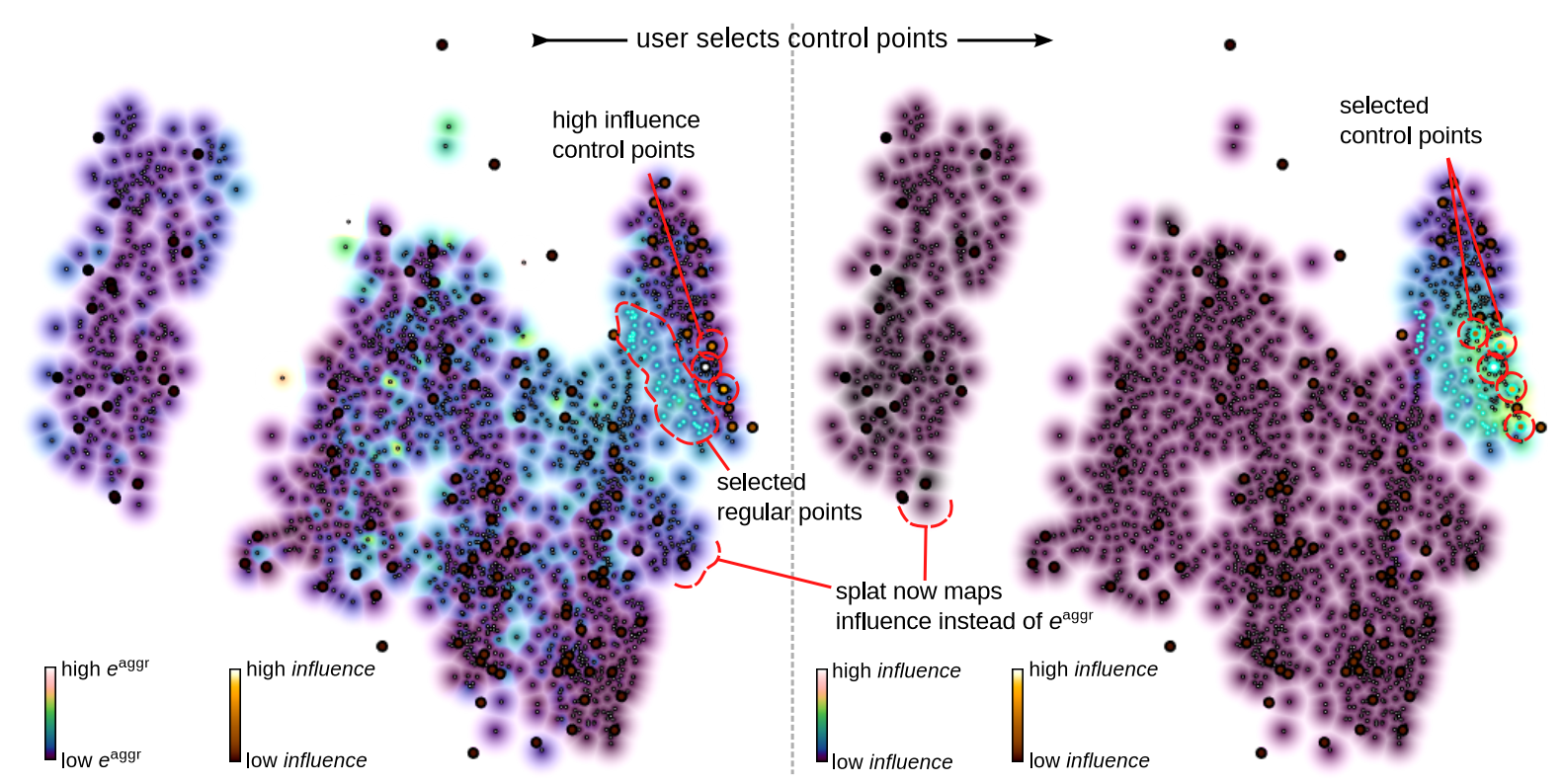

Source: Elaborated by the author.

Following that, we are faced with the question of which control points affect the region we are interested in. LAMP (subsection 2.2.3) defines the influence each control point $i$ has on a regular point $j$ using scalar weights $\alpha_{i j}$. These are defined as inversely proportional to the dissimilarity between the instance being projected and the instance represented by the control point, $\alpha_{i j}=\delta_{i j}^{-1}$. Based on $\alpha_{i j}$, we can color control points by summing the influence they have on the currently selected regular points. The control points with the largest influences will then stand out, enabling us to select only the most effective in our region of interest.

After selecting the control points we are interested in, the visualization is then updated, displaying the influence of the selected control points on all regular points. This information is useful because the user can now assess whether there are any other unwanted influences before even performing the manipulation. Since a control point might influence more points than those selected in the first step, displaying the global influence is useful in avoiding situations where unexpected behaviors might occur. The whole process is illustrated in Figure 9.

\subsubsection{Precise selections, brushing and linking}

The previous visualizations provide us with information to select control points that are effective in promoting the desired changes. Still, we may not be confident the selections we performed are the best available by just looking at the color of each glyph. It is presented in a way that allows us to visually infer which areas might be of our interest, but does not allow other information to be queried. As one example, it is not always trivial to select the control point with highest influence point among all points. 
Because of this, we complement the scatterplot view with two other views of bar plots, one for control points and the other for regular points. In these, each bar represents an instance, while their heights are proportional to the value associated with the point they represent. As an additional information, bars have the same color mapping as the glyphs in the scatterplot. Furthermore, the bars are always sorted in descending order, with the leftmost bar having the highest value and the rightmost, the lowest. These additional views, equipped with brushing and linking mechanisms with the scatterplot view, enables selections in whichever scenario is more convenient.

To illustrate this, we go back to our example. In the case of regions of interest, we performed the selection on the regular points we wanted the manipulation to reposition. Then, for selecting the control points with the largest influence on those, we can use the ordered bar plot, as shown in Figure 10. It shows how the brushing and linking mechanisms help us answer additional questions before manipulating the positions of control points. By using the bar plot to easily identify the control points, we reduce the amount of guessing and trial-and-error in the interactive process. Tasks such as identifying where are the points with the largest error or influence and determining how the error or influence is distributed are both facilitated.

\subsection{Highlighting unexpected behavior}

We also consider a complement to the splat visualization regarding the influence of control points. As previously mentioned, the splat visualization provides an overview of the error distribution over the projection. When interacting with a projection, we expect that nearby points are the ones we move when we select certain control points. Despite that, due to the high dimensionality of data, it is not always the case that all points influenced by a certain control point are nearby that point.

In these cases, the splat visualization might fail to highlight such "outliers", which, as a result of interaction, might be repositioned unexpectedly. As a tool for visualizing glyphs as being part of a field, it is not adequate to show those individual cases. A good illustration of this case is shown in Figure 9, where the projection on the right has a possibly unwanted influenced point that is hardly noticeable. Taking this into consideration, we designed an additional visualization on top of the scatterplot view for highlighting points that exhibit unexpected behavior when manipulating control points.

The first issue to address is quantitatively measuring a regular point as unreliable for an interaction. Intuitively, one would consider that points highly influenced by control points that are not nearby such control points should be an unreliable point. Thus, given the influence $\alpha_{i j}$ (subsection 2.2.3) a control point $i$ exerts on a regular point $j$, we measure the unreliability of such relation, denoted $\gamma_{i j}$, as

$$
\gamma_{i j}=\alpha_{i j}|| \mathbf{y}_{i}-\mathbf{y}_{j} \| .
$$


Figure 10 - Brushing and linking with the additional ordered bar plot allows more complex, informed selections and inspection.

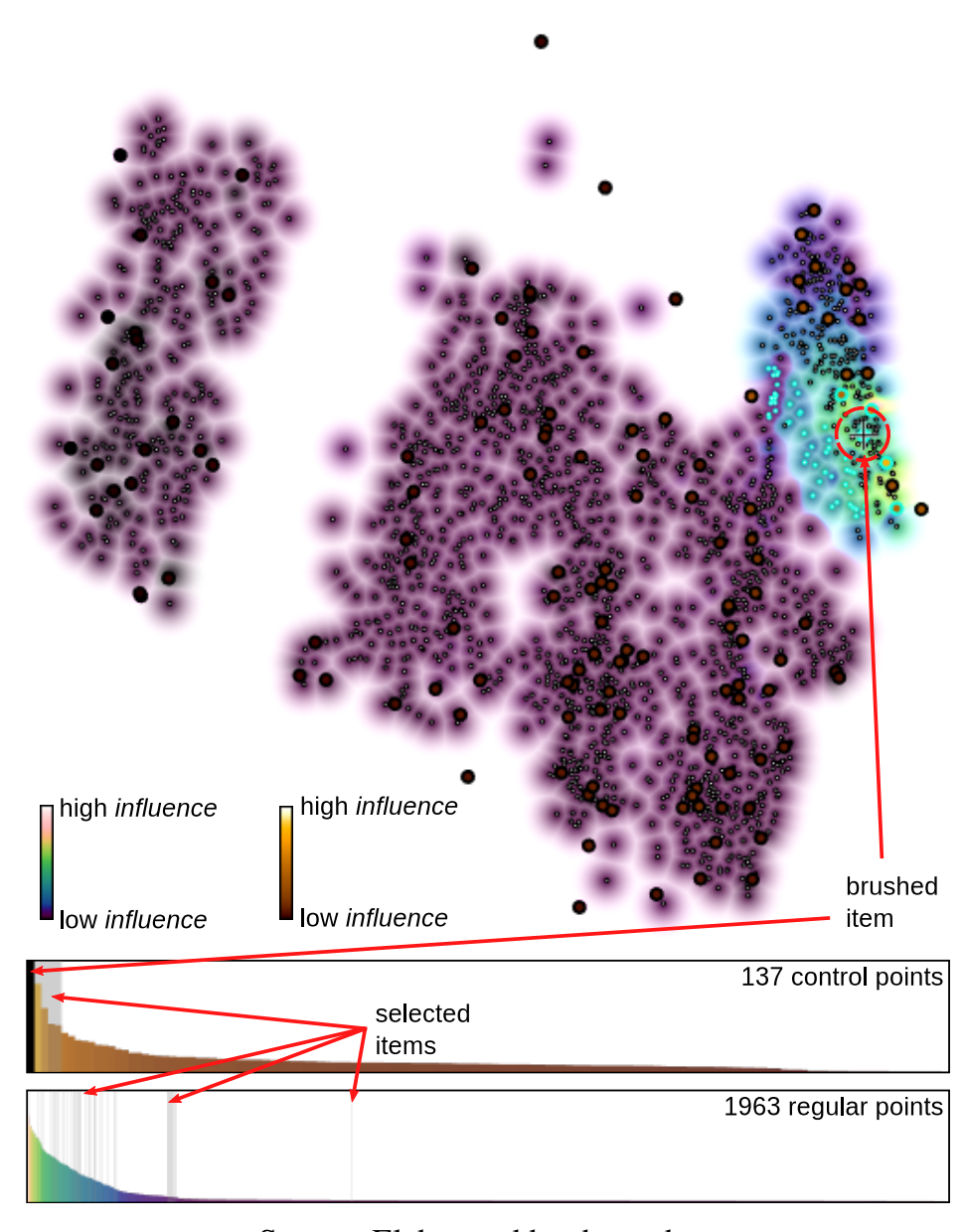

Source: Elaborated by the author.

One can see that $\gamma_{i j}$ has a large value is exactly when both the influence and distance are large, since we desire that a control point exert high influence to nearer points and low influence on farther points. Thus we take a percentage of the points with the largest values to assign as potentially unreliable. In our example, we use the $1 \%$ largest values. It should be noted that even when both influence and distance are small, this is not an issue, since with a small influence the control point cannot cause any change.

After computing the unreliability for based on a certain selection of control points, we draw lines connecting the unreliable regular points to the control points. An immediate issue with this approach would be the possibility of a high clutter caused by the lines drawn on top of the scatterplot. To address this, we use a fast graph edge bundling method (ZWAN; CODREANU; TELEA, 2016), reducing the visual clutter without compromising interactiveness with long computations. The final result for our example is shown in Figure 11. 
Figure 11 - Illustration of the graph bundling technique employed in the identifying unexpected behavior when manipulating.

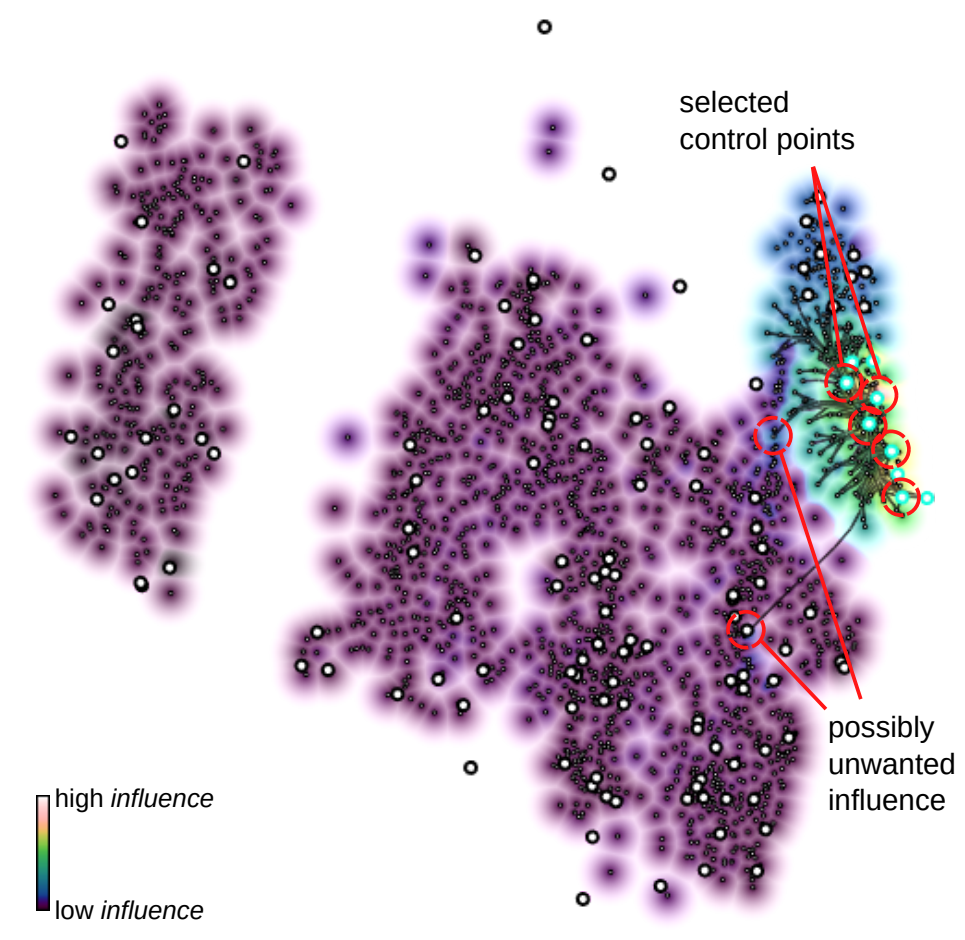

Source: Elaborated by the author.

\subsection{Explaining the manipulation}

After determining the control points we want to move using the provided visualizations, we perform the manipulation by directly moving the control points to a new desired position. Following this step, the map now changes: it is recomputed using the manipulated control points. Recall that, in our example, we wanted to separate a certain region of our map from the rest. In this section, we describe two ways of visually explaining the changes introduced by manipulating the control points.

\subsubsection{Changes in metrics}

A straightforward way of inspecting whether we achieved the desired result is to directly compute the difference between the aggregated errors in the new map and the previous map. One advantage of this method is its simple interpretation, as we reuse the same view used to convey the aggregated error. Moreover, we can also use the ordered bar plot to complement the visualization, as already discussed in section 3.1.

In this particular case, we also have to be careful with the color scale we employ. As we are looking at data where the difference in values can be more important than the actual values, a divergent color scale is more appropriate, so that positive and negative values have their own exclusive colors. In our case, increases in error values are red (positive) and decreases are blue 
Figure 12 - Difference in $e^{\text {aggr }}$ relative to previous map. The bar plot for control points is intentionally omitted as it contains no relevant information at this stage.

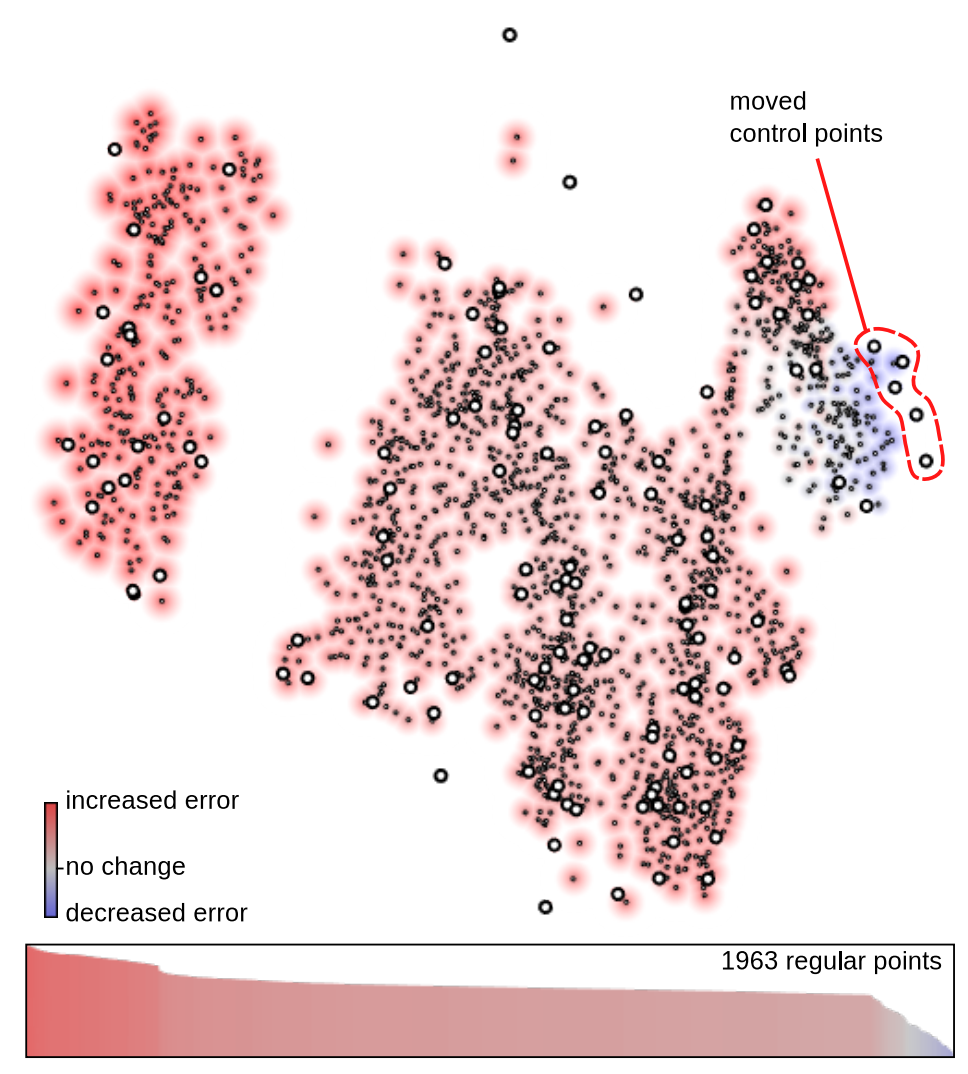

Source: Elaborated by the author.

(negative). In addition, values closer to zero become gray. This color scale naturally makes large differences noticeable, with the gray colors for less interesting values attracting less attention. The difference visualization is shown in Figure 12.

In the context of our example, this visualization reveals what happened when we moved the previously selected control points. Looking at the point positions, the manipulation caused the previously existing region between the main cluster and the rightmost cluster to be now better separated from the main cluster. A decrease in projection error for the regular points around the moved control points is also visible. Despite this, the visualization reveals that the error values in almost all other points increased to compensate. For a more complete analysis, however, one should also consider the now minimum and maximum values of differences, so that it is possible to evaluate whether the difference being shown is significant in terms of the original values.

\subsubsection{Changes in positioning}

Another visualization problem arises when we try to understand the repositioning that happens whenever a set of control points is manipulated. Since our main view is a scatterplot, and we usually have large number of elements (in our example, over 2000), approaches like streamlines are not appropriate, as they would result in a cluttered visualization and would be 
Figure 13 - Illustration of the animation interaction mechanism for visualizing changes in the map caused by manipulation.

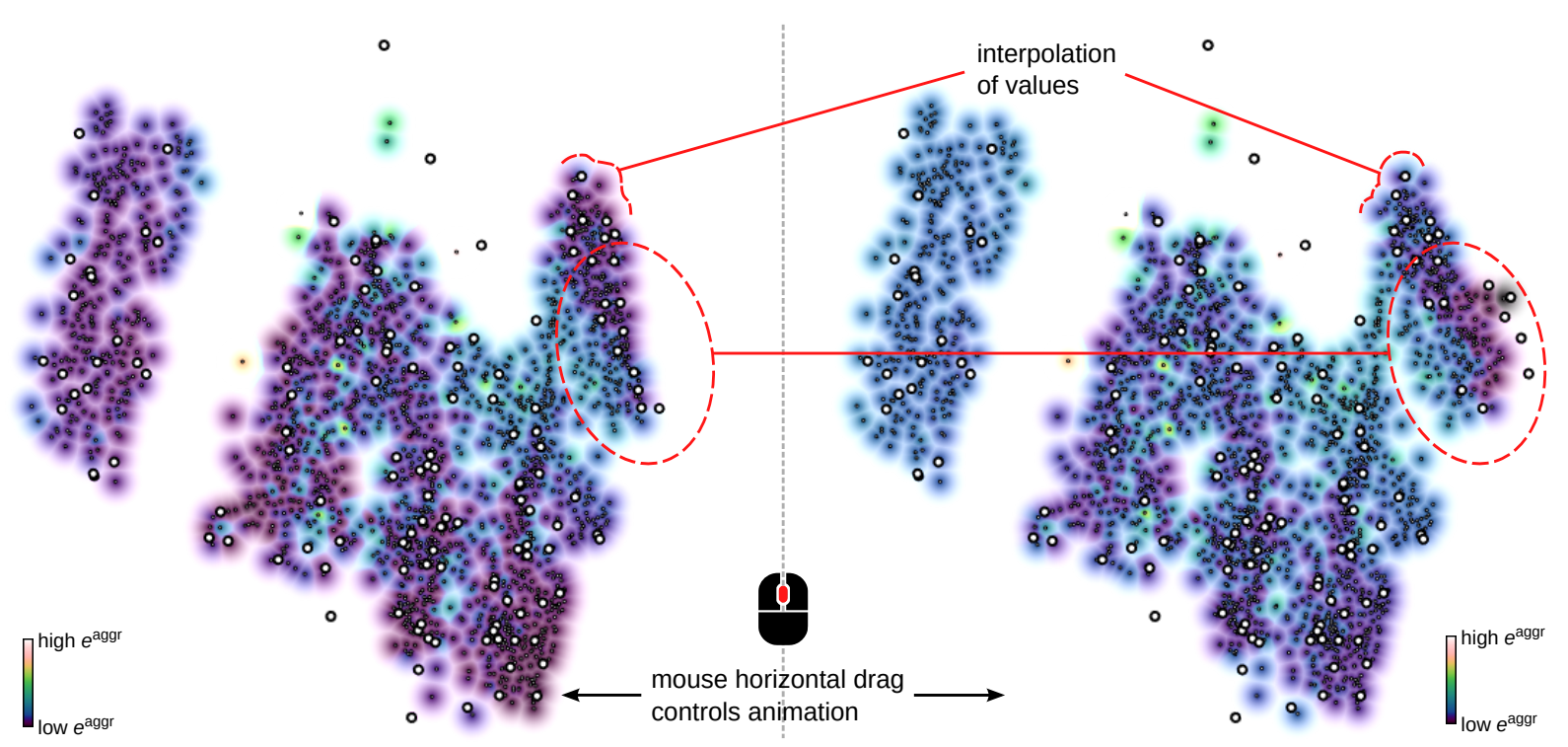

Source: Elaborated by the author.

in conflict with the visual representation for unexpected behavior (section 3.2). Moreover, the movement that happens is not always simple, as many interactive multidimensional techniques, such as LAMP, are nonlinear. Consequently, the use of animations is adequate, as it can convey movement while not cluttering the user view. One of the main drawbacks of animation, however, is that it can often lead to errors in interpretation (ROBERTSON et al., 2008). Because of this, we adopted a solution inspired by Hurter et al. (2014) that employs user-controlled animations, while reusing our scatterplot view.

This interaction mechanism works by defining a starting point along the horizontal axis, from which the animation timeline will be based upon. The starting point can be defined, for example, by middle-clicking somewhere in the scatterplot. Then, by dragging the mouse to the left, the user can "revert" in time the manipulation just performed. As the mouse moves, the animation occurs via a linear interpolation of the previous and the current maps, as the interpolation parameter is controlled by the mouse position along the horizontal axis. Whenever the mouse reaches the left edge of the scatterplot, the animation reaches its end and the map show is exactly the map from before the manipulation. The user is also free to move the mouse to the right while dragging, controlling the interpolation to go towards the current map instead. As an additional convenience, releasing the mouse button plays the animation forward in time, going towards the map obtained after the manipulation. An illustration of this interaction mechanism is shown in Figure 13.

This choice of approach, in favor of a traditional slider for example, results in a simpler user interface, keeping the user focused on the animation being displayed rather than how it is controlled. We also leverage the already available splat rendering by interpolating the error values 
between the two maps, changing the colors being displayed as a result. This, coupled with the ability to control the animation back and forth, enables even better analysis and explanation of the changes caused by manipulation. As we interpolate the values at the same time as the positions change, the animation conveniently highlights regions where the errors change significantly. It is also relevant to note that the interpolation is intended to understand the changes in positioning considering just the maps before and after the manipulation, not the maps "in between".

\subsection{Chapter conclusions}

In this chapter we presented and discussed a set of visualizations aimed at explaining interactivity in multidimensional projections. Their main purpose is to minimize the amount of trial-and-error inherent to this task. This is accomplished by providing the information needed at each step of the way. The visualizations are employed around four identified needs during interaction and manipulation in multidimensional projections.

First, the selection of the appropriate control points. Manipulation is based on moving control points, which in turn relies on selecting the appropriate ones. This is addressed by providing the user with information on which regions might be of interest to change interactively. Then, the user is informed of which control points would be the best candidates for this.

Second, the awareness of unexpected results in manipulation. The visualization aimed at this issue complements the previous visualizations with a way to highlight the possibility of unexpected results. In this way, the amount of trial-and-error in this process can be reduced by avoiding performing a manipulation that involves points with unreliable behavior in terms of expected results.

Third, the explanation of the changes in positioning caused by manipulation. After manipulating the appropriate control points, the technique does not guarantee that the user will attain the intended results. Moreover, due to the high dimensionality of the data under analysis and the non-linearity of the technique employed, the results are not always the same as expected. These difficulties are tackled via animations focused on providing easy control and a color map that displays changes in associated values.

One of the issues not addressed by these visualizations is the prediction of results before the manipulation takes place. This works in tandem with the objective of reducing trial-anderror in the process by providing information regarding the manipulation before it even takes place. Possible methodologies in this regard could involve approximation of results via local interpolations coupled with vector field visualization for displaying the direction of predicted movement.

Concerning the highlighting of unreliable points, one should note that choosing a fixed percentage of the highest values of unreliability can lead to unnecessary clutter. In Figure 11, we 
see that the influence is concentrated mostly nearby the control points. This shows that the $1 \%$ largest values contain points that will mostly behave within expectations. Consequently, the lines contain unnecessary information and contribute to clutter.

Another important issue is the number of elements and the resulting visual clutter. In principle, the number of elements a bar plot can accurately contain is equal to its width in pixels, since the minimum bar width is one pixel. Consequently, even though it can handle even twice that amount without significantly compromising selections, a number of elements much larger than that will still be a problem. The scatterplot will not help in this case either, as the large number of elements can make selections more difficult. 

Interactive multidimensional projection techniques (section 2.2) incorporate explicit user input in the computed map. This is accomplished by letting the user to manipulate a map of a subset of the dataset, indirectly dictating the layout of the overall map. Formally, given a dataset $\mathscr{X}$, let $\mathscr{X}^{\prime}$ be a sample of $\mathscr{X}$, with $\mathscr{X}^{\prime} \subset \mathscr{X}$ and $\left|\mathscr{X}^{\prime}\right|=c$. Also let $\mathscr{Y}^{\prime}$ be a $q$-dimensional map of $\mathscr{X}^{\prime}$, whose elements are called control points. We say that a multidimensional projection technique is interactive if $\mathscr{Y}^{\prime}$ is used by it to compute $\mathscr{Y}$, the $q$-dimensional map of $\mathscr{X}$.

Being interactive, these techniques are unique among other multidimensional data analysis techniques, which traditionally rely on parameter tuning to refine the mapping. Despite that, as of this writing, few studies (PAULOVICH; SILVA; NONATO, 2010; JOIA et al., 2011) have been conducted to analyze them from the interactivity perspective. Even those are still incomplete in terms of the types of interactions employed, focusing only on group separation and cohesion. Aside from the role of understanding data by interacting with it, these techniques enable novel visual analytics applications, such as the one introduced by Mamani et al. (2013). Consequently, it is fundamental for further exploiting such techniques in data analysis to properly assess and determine how effective is such manipulation. In this chapter, we describe our methodology to address the problem of quantitatively assessing the effectiveness of such manipulation.

\subsection{Defining and measuring effectiveness}

To address the aforementioned problem, consider the different forms of influence the control points have on the mapping. We organize them in three main forms: the choice of control points, the number of control points and their positioning itself.

The choice of control points determines which elements of $\mathscr{X}$ should be selected to be part of $\mathscr{X}^{\prime}$. There are specific strategies in the literature of multidimensional projections, but most pursue certain properties in the selection (PAULOVICH et al., 2008; FADEL et al., 2015). 
In contrast, Paulovich, Silva e Nonato (2010) present evidence that a sophisticated approach is not significantly different from random sampling. For completeness, we consider both on several sets of control points chosen by uniform sampling and a more sophisticated approach based on the $k$-medoids method (KAUFMAN; ROUSSEEUW, 1987).

Another form of influence is the number of control points, $c$. Usually, the adequate value of $c$ is determined using experimental solutions (PAULOVICH et al., 2008; PAULOVICH; SILVA; NONATO, 2010; JOIA et al., 2011; FADEL et al., 2015). Among these, the most common choices are either a percentage of the original number of items $n$ or a multiple of $\sqrt{n}$. Unless where otherwise stated, we use the multiple of $\sqrt{n}$ strategy for avoiding a large number of control points, which would hinder the experiments by leaving too few data items for techniques to handle.

Finally, the positioning itself, $\mathscr{Y}^{\prime}$, is another form of influence and the one we will mostly discuss throughout this chapter. As mentioned before, we are mainly interested in how effectively we can use $\mathscr{Y}^{\prime}$ to change $\mathscr{Y}$. Ideally, whenever a change is performed in $\mathscr{Y}^{\prime}$, we expect that $\mathscr{Y}$, computed after those changes, will reflect them. However, we cannot promptly infer whether this is the case, in general. Because of this, we need to establish what it means to be effective, relating effectiveness to whether the changes are faithfully reflected as expected. By using this definition in our analysis, we develop experiments that provide evidence as to whether interactive multidimensional projection techniques can properly generalize changes in the control points to the whole dataset. Thus, we define effectiveness of manipulation as follows.

Definition 4.1.1. Let $\mathscr{X}$ be a dataset, $\mathscr{X}^{\prime}$ be a sample of $\mathscr{X}$, and $\mathscr{Y}$ and $\mathscr{Y}^{\prime}$ be the $q$-dimensional mapping of $\mathscr{X}$ and $\mathscr{X}^{\prime}$, respectively. Suppose $\tilde{\mathscr{Y}}^{\prime}$ is the map of control points after the changes in positioning in $\mathscr{Y}^{\prime}$. Additionally, let

$$
m: \mathbb{R}^{p} \times \mathbb{R}^{q} \rightarrow \mathbb{R}
$$

denote a function that gives a number which evaluates the quality of $\mathscr{Y}$ relative to $\mathscr{X}$, according to some criteria. We say that a manipulation is effective when either

$$
m\left(\mathscr{X}^{\prime}, \tilde{\mathscr{Y}}^{\prime}\right)>m\left(\mathscr{X}^{\prime}, \mathscr{Y}^{\prime}\right) \Rightarrow m(\mathscr{X}, \tilde{Y})>m(\mathscr{X}, \mathscr{Y})
$$

or

$$
m\left(\mathscr{X}^{\prime}, \tilde{\mathscr{Y}}^{\prime}\right)<m\left(\mathscr{X}^{\prime}, \mathscr{Y}^{\prime}\right) \Rightarrow m(\mathscr{X}, \tilde{\mathscr{Y}})<m(\mathscr{X}, \mathscr{Y})
$$

holds.

The rationale behind definition 4.1.1 is based on the following observations. First, it is reasonable to expect that any change in control points should at least cause some change in the value produced by a quality measure. Specifically, if $\tilde{Y}^{\prime} \neq \mathscr{Y}^{\prime}$, then $m(\mathscr{X}, \tilde{Y}) \neq m(\mathscr{X}, \mathscr{Y})$, provided that $\tilde{\mathscr{Y}}^{\prime}$ was used to compute $\tilde{\mathscr{Y}}$ and $\mathscr{Y}^{\prime}$ to compute $\mathscr{Y}$. Second, overall visual layout changes caused by manipulation are straightforward to perceive, though observing definitive 
improvements in the mapping is not. By linking the effectiveness of manipulation to a number that evaluates some aspect of a mapping, we can assess its effects with better certainty. Third, while some quality measures produce values with known lower and upper bounds, many do not. This makes it difficult to perform comparisons between different mappings of the same dataset. Consequently, we circumvent this drawback by considering changes in a measure only in terms of it being greater (or less) than before the manipulation.

\subsection{Methodology}

In this section we describe how definition 4.1.1 is employed to study the effectiveness of manipulation. We developed a methodology for quantitatively assessing effectiveness, using it as the key concept for understanding user manipulation. Taking into account that there are many types of quality measures in the literature (SAMMON, 1969; ROUSSEEUW, 1987; PAULOVICH; MINGHIM, 2008; VENNA et al., 2010; MARTINS et al., 2014), such a methodology would not be complete if the many different aspects that they represent were not considered. Therefore, to perform this analysis, we designed a framework that contemplates a multitude of measures and techniques for consistently testing manipulation.

Manually performing an analysis of this nature, however, is not only susceptible to errors, but also a laborious task. Considering this, we also adopt manipulation procedures specifically tailored for each measure. With these, we overcome the challenges and pitfalls of manual manipulation while improving reproducibility. Creating a manipulation procedure for each measure, we intend to produce optimistic scenarios where an improvement by manipulation is available. In these scenarios, each technique is given a reasonable margin of improvements for generalizing them to the whole dataset. Hence, the optimistic essence of this methodology allows us to observe, in a measurable sense, the effectiveness of manipulation. Figure 14 depicts all the stages that compose this methodology, which we detail below.

Essentially, in this framework we consider two analyses for the manipulation. In the first, we consider uniformly sampled sets of control points. This scenario evaluates a technique's capability of being influenced by manipulation in a more broad sense. Using the same technique to initialize a projection for each set of control points, we perform manipulations that improve several different measures. Then, the results are compared using the measures extracted from before and after the manipulation. These measures are compared in two ways: using the average measure before and after visualization and the confidence intervals for the difference between the measures after manipulation and before.

An example of how we present these results is shown in Figure 15. A few important details are worth highlighting in this illustration. For the confidence interval results, whenever an interval contains zero, we do not have enough evidence to assess whether the improvements were relevant. On the other hand, intervals that do not contain zero represent statistically effective 
Figure 14 - Overview of the methodology for effectiveness analysis. We employ two approaches, one with a number of different uniform samplings that will be used as control points and other focusing on gradual improvement. The first encompasses broader scenarios, while the second focuses on the behavior as the manipulation improves a certain aspect of the positioning of control points.

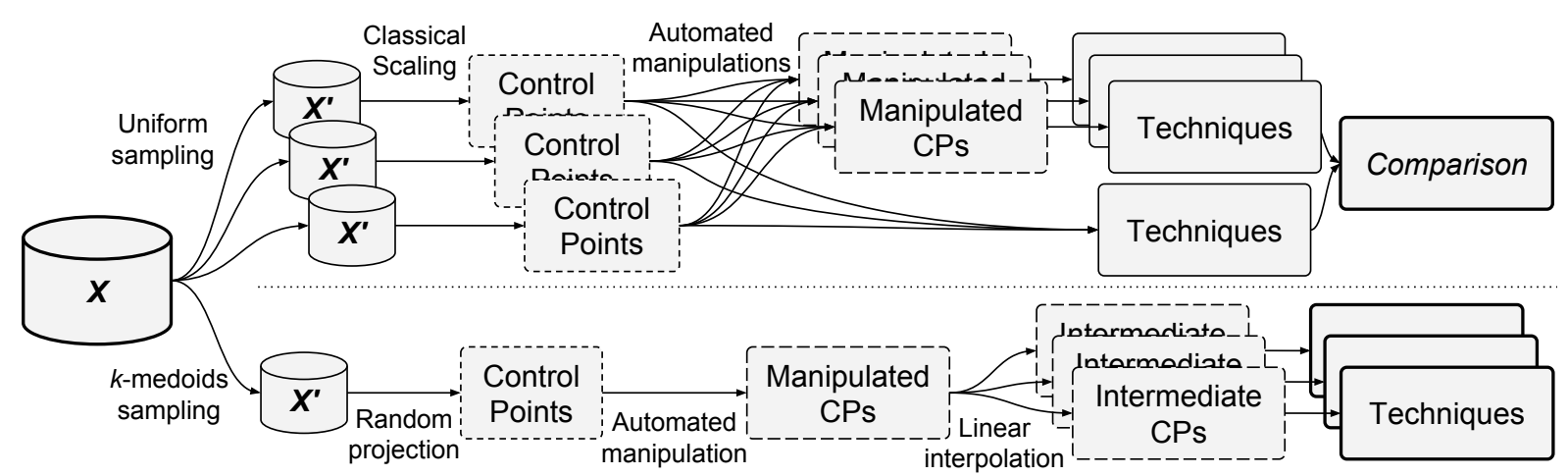

Source: Elaborated by the author.

Figure 15 - Details of how we present our results for many sets of control points. The confidence intervals (left) allow a statistically significant assessment of whether the difference between the improvement and the original measures is positive. The average values (right) are used for an overview of the behavior, allowing comparisons between many different combinations of techniques and datasets.

confidence interval for the difference:

after minus before manipulation

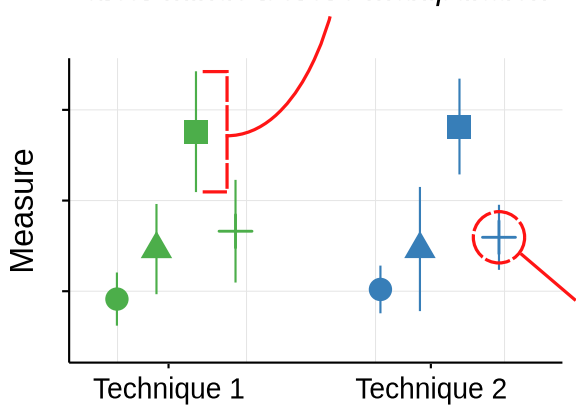

average measure value

(after manipulation)

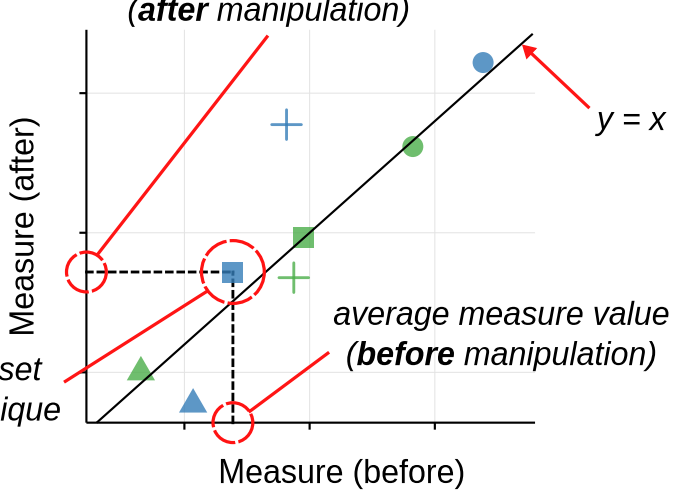

Source: Elaborated by the author.

manipulations. For the average measures, we have a simpler visualization that shows more details while being less statistically rigorous. In this case, we are interested in how points are positioned relative to the $y=x$ line: points above the line represent increased measure values after manipulation while points below the line represent decreased measure values.

In the second analysis, we start with a representative selection of control points using a $k$-medoids strategy. In this case, we intend to observe the behavior of gradual improvement in each type of manipulation. By starting from a random projection of control points $Y_{r}^{\prime}$, we linearly interpolate towards an improved control point positioning $Y_{f}^{\prime}$ with

$$
Y^{\prime}=(1-\alpha) Y_{r}^{\prime}+\alpha Y_{f}^{\prime}, \quad 0 \leq \alpha \leq 1 .
$$

These several stages are computed for values of $\alpha$ ranging in fixed steps from 0 to 1 . Then, each $Y^{\prime}$ computed with Equation (4.2) is used as input to the techniques. 
Figure 16 - Details of how we present our results for a set of control points chosen using the $k$-medoids strategy. Since we are interested in the evolution of measures, we connect related observations of the same dataset and technique, allowing us to see how the measures change in the full map as we improve them in the map of control points.

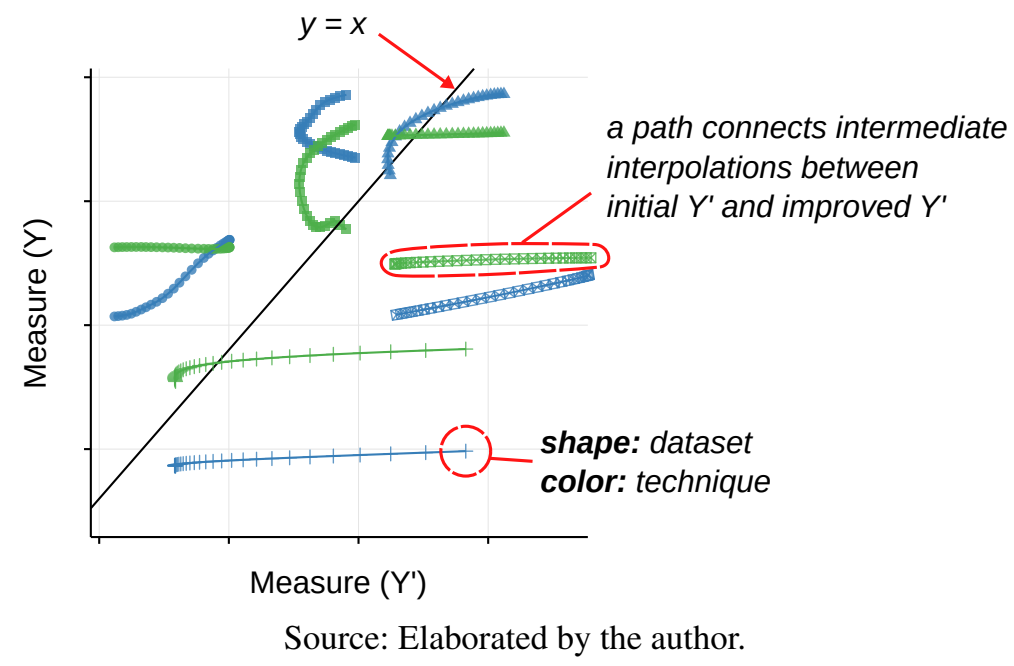

Figure 16 contains an illustration of how we present these results. In such a scatterplot, points whose projections are computed using the same $Y_{r}^{\prime}$ and $Y_{f}^{\prime}$ in the linear interpolation are connected with a line. Thus, each path of points represents the evolution of a measure as we go from a random projection towards a map that maximizes a certain measure as much as possible. Note that in this case, as opposed to Figure 15, the horizontal axis represents values of measures with respect to $Y^{\prime}$. Paths with a relatively positive "slope" represent the best case scenario: a measure improves for the full map as it improves with the positioning of control points. Contrarily, negative "slopes" represent measures that degrade as the control points mapping improves. Horizontal paths are cases where the manipulation has very little effect on the outcome.

\subsubsection{Quality measures}

As mentioned before, the use of various measures is necessary to evaluate the effectiveness of manipulation in different aspects. As a single measure would only illustrate a single type of manipulation, this could hinder our analysis.

\subsubsection{Silhouette coefficient}

The silhouette coefficient (ROUSSEEUW, 1987) measures the compactness and separation groups of points in $\mathscr{Y}$ based on their class labels. Originally designed to evaluate clustering algorithms, it can be employed in our context to evaluate how well $\mathscr{Y}$ can represent the different classes in the data.

Suppose the label of $\mathbf{y}_{i}$ is $l^{\prime}$. Let $a(i)$ be the average distance between $\mathbf{y}_{i}$ and each other point whose label is $l$. For every label $l^{\prime}$ not equal to $l$, consider the average distance between $\mathbf{y}_{i}$ and each each point whose label is $l^{\prime}$. Let $b(i)$ be the smallest of such averages. The silhouette 
coefficient $s(i)$ of $\mathbf{y}_{i}$ is defined as

$$
s(i)=\frac{b(i)-a(i)}{\max \{a(i), b(i)\}} .
$$

In this measure, being well grouped is a combination of both having small average distance between points of the same class while at the same time being distant from points of other classes. Therefore, compact, isolated clusters of instances have large silhouette values. The silhouette coefficient of $\mathscr{Y}$ is the average silhouette value over the whole dataset. Since $-1 \leq s(i) \leq 1$ for $1 \leq i \leq n$, we have that the silhouette coefficient is always in the range $[-1,1]$.

It is worth noting that classes are usually assigned by a human. Hence, they are not only susceptible to error but also a subjective assignment not entirely representable by the data. As a result, class separation in $\mathbf{y}_{i}$ dimensional settings is not always possible.

\subsubsection{Sammon's stress}

Another measure interesting to our analysis is stress (SAMMON, 1969). It quantifies the distortion in $\mathscr{Y}$ relative to $\mathscr{X}$ in terms of representing the original dissimilarities as the distance between items in the map. Formally, it measures how $\delta\left(\mathbf{x}_{i}, \mathbf{x}_{j}\right)$ is different from $\left\|\mathbf{y}_{i}-\mathbf{y}_{j}\right\|$ for every $1 \leq i \leq j \leq n$. The stress $\sigma$ of $\mathscr{Y}$ relative to $\mathscr{X}$ is the same as the error function, given by Equation (2.11), used in the Sammon's Mapping optimization (subsection 2.1.4).

As opposed to the silhouette coefficient, it measures how faithfully the map represents the dissimilarities of the original data, not relying on semantic information such as labels. Therefore, it is also an important aspect to include in our analysis.

\subsubsection{Neighborhood preservation index}

We evaluate the representation of neighborhood structures using the neighborhood preservation index (PAULOVICH; MINGHIM, 2008). Let $k \mathrm{NN}\left(\mathbf{x}_{i}\right)$ and $k \mathrm{NN}\left(\mathbf{y}_{i}\right)$ be the sets of indices of the $k$ nearest neighbors of $\mathbf{x}_{i}$ and $\mathbf{y}_{i}$, respectively. The neighborhood preservation index is the proportion of indices of the $k$ nearest neighbors of $\mathbf{x}_{i}$ that are also the $k$ nearest neighbors of $\mathbf{y}_{i}$ in the map,

$$
\mathrm{NP}_{k}(i)=\frac{\left|k \mathrm{NN}\left(\mathbf{y}_{i}\right) \cap k \mathrm{NN}\left(\mathbf{x}_{i}\right)\right|}{\left|k \mathrm{NN}\left(\mathbf{x}_{i}\right)\right|} .
$$

As this measure represents a proportion of the original neighborhood that is preserved in the map, we have $0 \leq \mathrm{NP}_{k}(i) \leq 1$. Additionally, since having more of the original neighborhood preserved is better, larger values represent better neighborhood representation. To evaluate the whole map, we average $\mathrm{NP}_{k}(i)$ over all points.

\subsubsection{Smoothed precision and smoothed recall}

Smoothed precision and smoothed recall are generalizations of the traditional information retrieval concepts of precision and recall (BAEZA-YATES; RIBEIRO-NETO, 2011). They were 
Figure 17 - An example of automated manipulation (NEWSGROUPS dataset; see chapter 5 for details). On the left, the map computed using the initial projection of control points. On the right, the map computed using control points manipulated to improve the silhouette coefficient. The control points used to compute the maps are depicted at the bottom-left insets of each map. Glyphs are colored according to the label of the instance they represent.

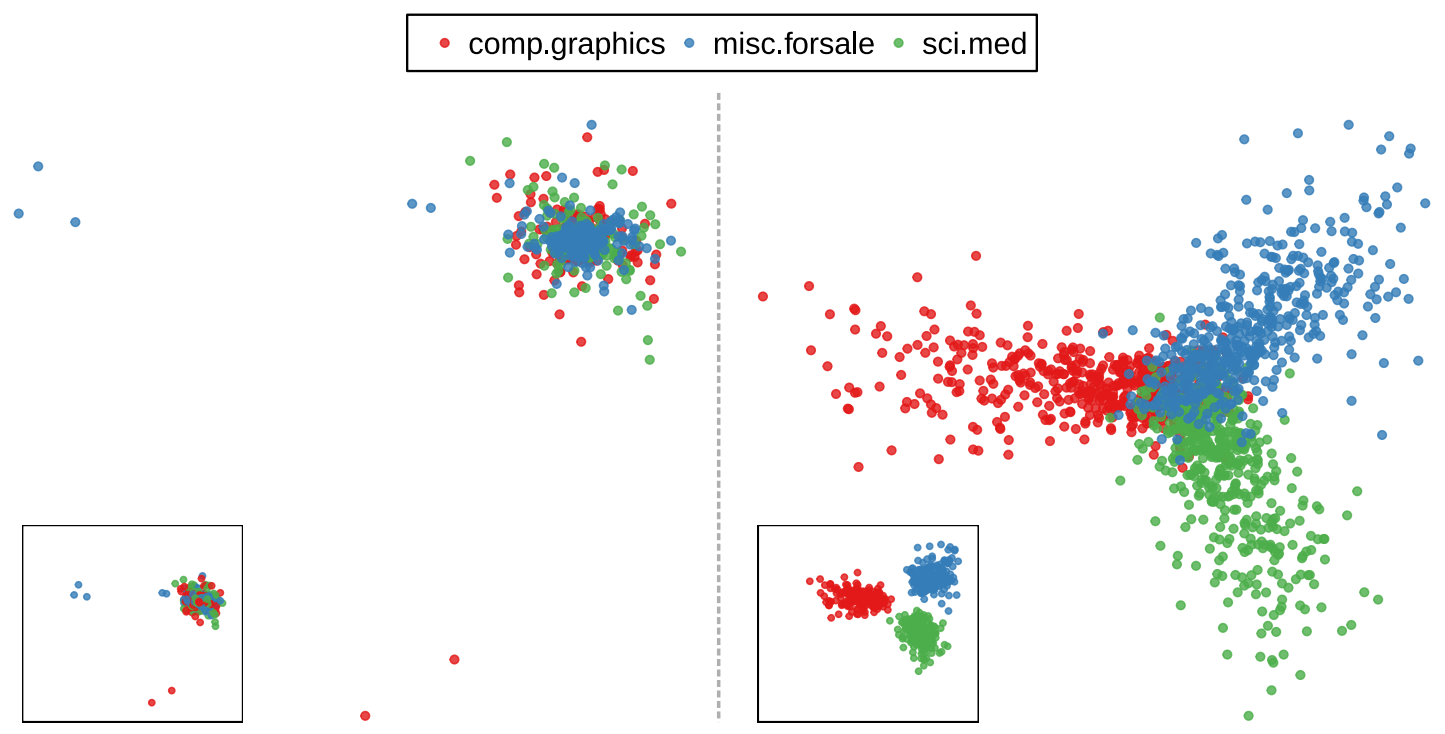

Source: Elaborated by the author.

introduced by Venna et al. (2010) as the foundations for the NeRV technique (subsection 2.1.6). In the context of visualizing high dimensional data using dimensionality reduction, smoothed precision and smoothed recall measure how well a certain map is capable of "retrieving" the original neighbors whenever the user inspects some point $\mathbf{y}_{i}$. This makes them interesting to add to our analysis, as the other measures are more focused on either preservation of distances, in the case of stress or rank preservation of distances, in the case of neighborhood preservation.

The smoothed precision and smoothed recall of $\mathbf{y}_{i}$ are given by $\operatorname{KL}\left(Q_{i} \| P_{i}\right)$ and $\operatorname{KL}\left(P_{i} \| Q_{i}\right)$, respectively, with $P_{i}$ defined in Equation (2.19) and $Q_{i}$ defined in Equation (2.20). To evaluate a map, we average the smoothed precision and smoothed recall over all points. A drawback of using smoothed precision and smoothed recall is that they are difficult to compare across different datasets, as the values obtained are not bounded.

\subsubsection{Automated manipulation procedures}

The measures previously mentioned evaluate different aspects of each mapping. As such, the manipulation required to improve them will have different objectives, which will be useful in quantifying the capability of each technique of generalizing various types of manipulations. Then, by comparing the attained maps before and after the manipulation, we can assess from an effectiveness perspective, how useful it was in creating a better representation of the data. In Figure 17, we provide an example of how the automated procedure is used to improve a measure, resulting in positive changes on the final projection. 
Below we describe the procedure employed to improve each measure.

Silhouette coefficient: We use the transformation introduced by Schaefer et al. (2013) to improve the silhouette coefficient. It is tailored exactly for separating and compacting clusters of points in a map using their class labels. Essentially, the technique adds more attributes to instances based on their class. Those new attributes increase the dissimilarity between instances of different classes and decrease the dissimilarity between those of the same class. Consequently, group separation and compactness is improved. We use classical scaling (subsection 2.1.2) to compute the new positions with these dissimilarities.

Stress: This measure is directly optimized using Sammon's Mapping (subsection 2.1.4). Since it optimizes the measure we are interested in seeing improved, we can simply use it to attain the desired manipulation.

Neighborhood preservation index: This measure is not as simple to optimize as the other measures, since as of this writing there are no methods that directly optimize it. Because of this, we use the t-SNE technique (subsection 2.1.6), which is known to produce maps with high neighborhood preservation when compared to many other techniques (FADEL et al., 2015).

Smoothed precision and smoothed recall: Both of these measures can be directly optimized using the NeRV technique (subsection 2.1.6). This technique allows us to separately optimize them by setting its $\lambda$ parameter appropriately. If $\lambda=0$, the technique optimizes the smoothed precision. On the other hand, if $\lambda=1$, it optimizes the smoothed recall.

\subsection{Chapter conclusions}

In this chapter we introduced the concept of effectiveness with respect to interactivity in multidimensional projection techniques. This concept guided the design of our experimental framework for understanding the limitations of manipulation. It was therefore central in our approach to tackling the problem of determining whether interactions with these techniques can indeed produce better representations of data as a result.

There are some limitations in the experimental framework. Since we don't test control point selection strategies, the full potential of manipulation could be hindered by not selecting the appropriate set of control points for each technique. We believe that several sets of uniformly randomly chosen control points are representative enough, but provide no guarantees for that. Consequently, unfavorable results could be attributed to suboptimal selections. Determining this set is a challenging issue, as most techniques in our study were not designed with clear specifications of what the optimal set of control points is. Additionally, a theoretical study, in favor of an experimental one, on interaction with multidimensional projection techniques could lead to new insights on how to better exploit interactivity. 


\section{EXPERIMENTS AND RESULTS}

\subsection{Experimental protocol}

We put our methodology to test in a series of experiments performed on a variety of datasets and techniques. In this section, we detail the experimental protocol, datasets and techniques used while performing the experiments.

\subsubsection{Techniques}

The techniques chosen for our experiments are LAMP (subsection 2.2.3), PLMP (subsection 2.2.2), LSP (subsection 2.2.1) and Pekalska's approximation (subsection 2.1.4). In Pekalska's approximation, we provide the manipulated control points as the initial sample of instances for computing the final mapping. This way, we can extend it to be interactive, including it in our tests and have a more diverse set of techniques to analyze the behavior from. Additionally, LAMP is tested twice in our experiments. That is because it can optionally project points considering only a certain number of nearest control points. We use two strategies: one considering all control points and one considering only the $25 \%$ nearest. Throughout this chapter, we call the first case LAMP-g and the second LAMP-25\%. As a benefit, we can also compare how this additional local behavior is relevant for effectiveness.

As for the other techniques, LSP has its neighborhood size set to $k=15$. Due to the PLMP formulation, we are unable to use less than $p$ control points for any dataset. Thus, whenever $c<p$, we use $p$ control points for all techniques, where $c$ is the number of control points obtained from the standard strategy we employ, described in subsection 5.1.4. This is the case for FACES, NEWSGROUPS and MNIST datasets. Pekalska requires no other parameters besides the input dissimilarities. We use the Euclidean distance normalized to have unitary mean as our dissimilarity function in all experiments. 
Table 1 - Summary of the datasets used in our experiments for evaluating effectiveness in ascending order of number of instances. Numbers shown for each dataset are not necessarily the same as it is provided by its source. Refer to Appendix B for more details regarding data preparation and preprocessing in our experiments.

\begin{tabular}{rcccll}
\hline Name & Instances $(n)$ & Dimensions $(p)$ & Labels & Source \\
\hline WDBC & 569 & 30 & 2 & Lichman (2013) & \\
FACES & 698 & 256 & - & Tenenbaum, Silva e & Langford (2000) \\
& & & & Lichman (2013) & \\
NEWSGROUPS & 1492 & 512 & 3 & Lecun et al. (1998) \\
MNIST & 1934 & 512 & 10 & Lichman (2013) \\
SEGMENTATION & 2086 & 18 & 7 &
\end{tabular}

Source: Elaborated by the author.

\subsubsection{Datasets}

The datasets are summarized in Table 1. They were chosen as being representative of different types of problems under analysis using multidimensional projections. Although most of the datasets are related to visual (image) data, they have sufficiently different features to justify this. For example, both the FACES and MNIST datasets consist of raw image data. However, the FACES dataset has no labels, as it is commonly used to evaluate manifold learning techniques (TENENBAUM; SILVA; LANGFORD, 2000), whereas the MNIST dataset is targeted towards classification. Refer to Appendix B for more details regarding data preparation and preprocessing in our experiments.

As some measures and techniques might also require dissimilarities as input, whenever that is the case, we use the Euclidean distance as our dissimilarity function. Furthermore, we also normalize all distances, including distances between points in the $q$-dimensional space (visual space), to have unitary mean. This helps us avoid possible issues with different scales of distances across techniques.

\subsubsection{Measures}

Some measures we chose to conduct our study requires parameters for evaluating the maps, which we set as follows. The neighborhood sizes we used on the measures was set to be the minimum between $\sqrt{n}$ and $5 \%$ of $n$. Thus, the neighborhood size for the neighborhood preservation index and the perplexity on smoothed precision and recall were all set this way.

The techniques used to improve some of the measures also require parameters to be set, which we detail here. The perplexity of t-SNE and NeRV were set to the neighborhood size in our experiments, which is the maximum value between 15 and $5 \%$ of the number of instances. Additionally, the $\lambda$ parameter of NeRV for optimizing smoothed precision and smoothed recall was set to $\lambda=0.01$ and $\lambda=0.99$, respectively. All other parameters of both t-SNE and NeRV are the recommended defaults by the authors. 


\subsubsection{Control point selection and mapping}

As previously mentioned, we use both a uniform random sampling strategy and a $k$-medoids approach for picking control points. Both methods use $3 \sqrt{n}$ control points or $p$, whichever is larger, since PLMP requires at least $p$ control points. Particularly for the random sampling, selections containing few representative points, such as having many similar points, can happen. To account for this, we perform 30 control point selections for each dataset. These control points are then mapped to two dimensions using classical scaling (subsection 2.1.2). As classical scaling is a linear technique, this strategy allows a reasonable margin to improve the various measures under analysis, since all of them are improved using nonlinear techniques.

As for the $k$-medoids approach, it is inspired by the control point selection methods present in some other techniques (PAULOVICH et al., 2008; FADEL et al., 2015). The reasoning behind this method is that, since we want better representative points, we should select as much "central" instances as possible, as they represent the many possible groups of instances in the dataset (FADEL et al., 2015). Thus, we set $k$, the number of medoids, to be equal to the number of control points, and use the computed medoids as the control points. These control points are then projected using a random projection (ACHLIOPTAS, 2001) so that we have the most headroom to observe gradual improvements.

\subsection{Results and discussion}

We start with an overview of the results containing the averages for measures over all control point selections, for each technique and dataset. Scatterplots of the data are shown in Figure 18. The scatterplots are annotated with a $y=x$ line, faciliting visual inspection: a measure increased when points are above the line, and decreased when below. These results illustrate the general behavior of techniques regarding each type of manipulation.

The first prominent results worth discussing are related to smoothed precision and recall. Surprisingly, none technique could consistently improve these measures, despite their solid foundation on a well-defined task and a direct optimization employed in the manipulation. This indicates that manipulations that improve the retrieval of elements for visualization, as defined by Venna et al. (2010), could not be captured by the techniques.

Another noticeable behavior is related to stress. As seen in Figure 18, every technique on every dataset is either close or far below the diagonal line. Since stress has been directly optimized using Sammon's Mapping, we have a guarantee that it was indeed improved by the automated manipulation. Despite this, results show that, on most cases, either little to no improvement (points close to the line) or significant improvements happened in the quality of the map.

There are some possible explanations for this behavior. The cases where stress has 
significantly improved involve mostly the MNIST, NEWSGROUPS and FACES datasets. These datasets are the most complex and high dimensional, justifying their poor stress values for all techniques. As a result, this gives the manipulation procedure more headroom for improvement. While this is true, only LSP and Pekalska's techniques had significant improvements. In the Pekalska's, since it was originally designed as an interpolation method for Sammon's Mappping, it might be more susceptible to carrying over the improvements to the whole map. LSP, on the other hand, is highly dependant on the control points for positioning. Since it tends to place points inside the convex hull formed by the nearby control points, the layouts attained usually respect the overall geometry of the control points.

The silhouette coefficient displayed improvements in almost all datasets and techniques. The NEWSGROUPS dataset is noticeably above the diagonal line for all techniques, except PLMP. As illustrated in Figure 17, the initial layout obtained by classical scaling is very cluttered. However, after manipulation, the Pekalska's technique attained a much better representation of classes. On the other hand, we see the opposite happened to the MNIST dataset, where only Pekalska's technique achieved some improvement. This is not necessarily positive for this technique, as it is probably due to having more headroom for improvement, as the original silhouette value was lower than all other techniques.

Moving our focus towards techniques, PLMP has little to no improvement on most measures and datasets. This is most likely due to the fact that, as shown by Paulovich, Silva e Nonato (2010), PLMP can be seen as a generalization of PCA. Whenever the linear transformation cannot properly represent the control point mapping, the resulting projection is not improved. Since PCA is equivalent to classical scaling when using the euclidean distance, as is our case, this could mean that PLMP is capable of some improvements, but the general behavior is similar to that of classical scaling.

Other noteworthy results are LSP and Pekalska's technique behavior in neighborhood preservation, silhouette and stress. While not always showing improvements, these techniques consistently show improvements whenever some other technique also show improvement, usually outperforming them. This is a surprising result for Pekalska's technique, as it consists of a simple linear approximation of the transformation between the original distances and the initial positions. LSP, however, as previously mentioned, is known for preserving the global geometry of the projection, and these results corroborate that fact.

Aiming at providing statistically meaningful results, we also show how each technique behaved in each dataset with respect to the difference in measures in terms of confidence intervals. These results detail and complement the results in Figure 18. To determine the confidence intervals, we subtract the measures obtained before the manipulation, $m(\mathscr{X}, \mathscr{Y})$, from the measures obtained after, $m(\mathscr{X}, \tilde{\mathscr{Y}})$. The computed $95 \%$ confidence intervals are shown in Figure 19 as line plots. Whenever the confidence interval of this difference does not contain zero, 
we can use definition 4.1.1 to statistically assess whether a manipulation was effective, since

$$
m(\mathscr{X}, \tilde{\mathscr{Y}})-m(\mathscr{X}, \mathscr{Y})>0 \Leftrightarrow m(\mathscr{X}, \tilde{Y})>m(\mathscr{X}, \mathscr{Y}) .
$$

The confidence intervals confirm the surprising behavior with smoothed precision and smoothed recall that was previously discussed. With smoothed precision, no technique could achieve a significant improvement, with the only exception being the SEGMENTATION dataset. In this case, LAMP-25\% achieved a significant improvement, while all other techniques exhibited a large decrease. In the case of smoothed recall, the scenario is not very different. With the exception of Pekalska's technique in the MNIST dataset, no technique achieved a significant improvement in smoothed precision or smoothed recall, and those which did were only in a single dataset. Instead, there were also many cases of decrease, with LSP in the MNIST dataset being the most noticeable. Corroborating to this idea, we see in Figure 20 that the behavior in smoothed precision is highly unstable, while smoothed recall is mostly stagnant in improvement.

Furthermore, results on stress and silhouette also confirm previous observations. The silhouette manipulation is effective on most techniques and datasets, with the exception of PLMP and MNIST. Additionally, Pekalska's technique along with LSP generally outperform other techniques on both measures. Particularly on stress, MNIST has the largest improvements with the best performing techniques, while LAMP-25\% has the worst performance, which was not as visible before due to cluttering in the previous results. In the LAMP-25\% case, we see that the "discontinuities" introduced by the increased local mapping is detrimental to stress, achieving results even worse than PLMP, the only technique that performs poorly, with respect to effectiveness, on all measures.

Regarding neighborhood preservation, a behavior similar to that of the silhouette coefficient is observed. Not all of those were visible in the previous results, also due to cluttering on smaller neighborhood preservation values. Although even PLMP showed some noticeable improvements, it also underperformed compared to other techniques. Despite this, it was the only technique which did not reduce the neighborhood preservation on the WDBC. This could be explained by the automated manipulation procedure we adopted, which is based on t-SNE. In our experiments, we noticed that t-SNE tends to perform poorly in small datasets, producing poor maps. Since WDBC is already small and we also employ t-SNE only on the control points, this could explain the poor results and corroborates the idea that PLMP is less prone to be affected by manipulation than other techniques. Despite this, the neighborhood preservation manipulations were effective in the other cases.

Focusing on the results in Figure 20, in most cases the potential for improvement is leveraged by the automated manipulation, leading to a better map overall. However, we must also consider that by starting from a very low measure, we expected to have an observable improvement. Particularly distinct is the behavior on the silhouette coefficient for the SEGMENTATION and NEWGROUPS datasets that many techniques were effective on. In the case of 
SEGMENTATION, we see that the measure does not always improve in terms of the sample, as the linear interpolation produces some worse configurations in terms of this measure, but the observed results in the full map are constantly improving. Conversely, we see the opposite in the NEWSGROUPS dataset, as the measure consistently improves in the control points' mapping but not in the full map. This might suggest that this methodology of linearly interpolating from two configurations is not the most adequate for this type of analysis. One other possible explanation is that the selection of control points might affect these datasets differently. While the prior assumption that the hierarchical clustering approach leads to a good set of control points works for SEGMENTATION, it was not a good choice for NEWGROUPS, making manipulation have almost no effect in the results.

\subsection{Chapter conclusions}

The experiments were conducted on five datasets with varied characteristics and five techniques, including two variations of one of them. Each technique and dataset combination was run several times with different control points, encompassing a wide range of scenarios. By using a series of automated manipulation procedures, we tested several types of manipulations with the intent of observe which kinds of manipulations the techniques were capable of carrying over to the full map.

The attained results indicate that PLMP, due to its inherent simplicity is in general not capable of incorporating improvements provided by user manipulation. Moreover, the only types of manipulation that proved effective were the ones improving the neighborhood preservation index and the silhouette coefficient. The only techniques that consistently showed noticeable improvements were LSP and Pekalska's. This result is surprising since Pekalska's technique uses a linear approximation to attain a transformation from dissimilarities into $q$-dimensional coordinates, whereas LSP uses a large linear system for mapping points close to their neighbors.

Smoothed precision and smoothed recall, despite their rigorous background and meaningful formulation, were not successfully incorporated as a manipulation by any technique, with a single exception in a single dataset in both. Surprisingly, the results were often worse after manipulation. 
Figure 18 - Scatterplots of the average measurements over all control point selections, before and after manipulation. The diagonal line $(y=x)$ is displayed as a reference for increase and decrease in the measures. Since the FACES dataset has no labels, it is not shown in the silhouette coefficient scatterplot. The highlighted area denotes an improvement in the measure.
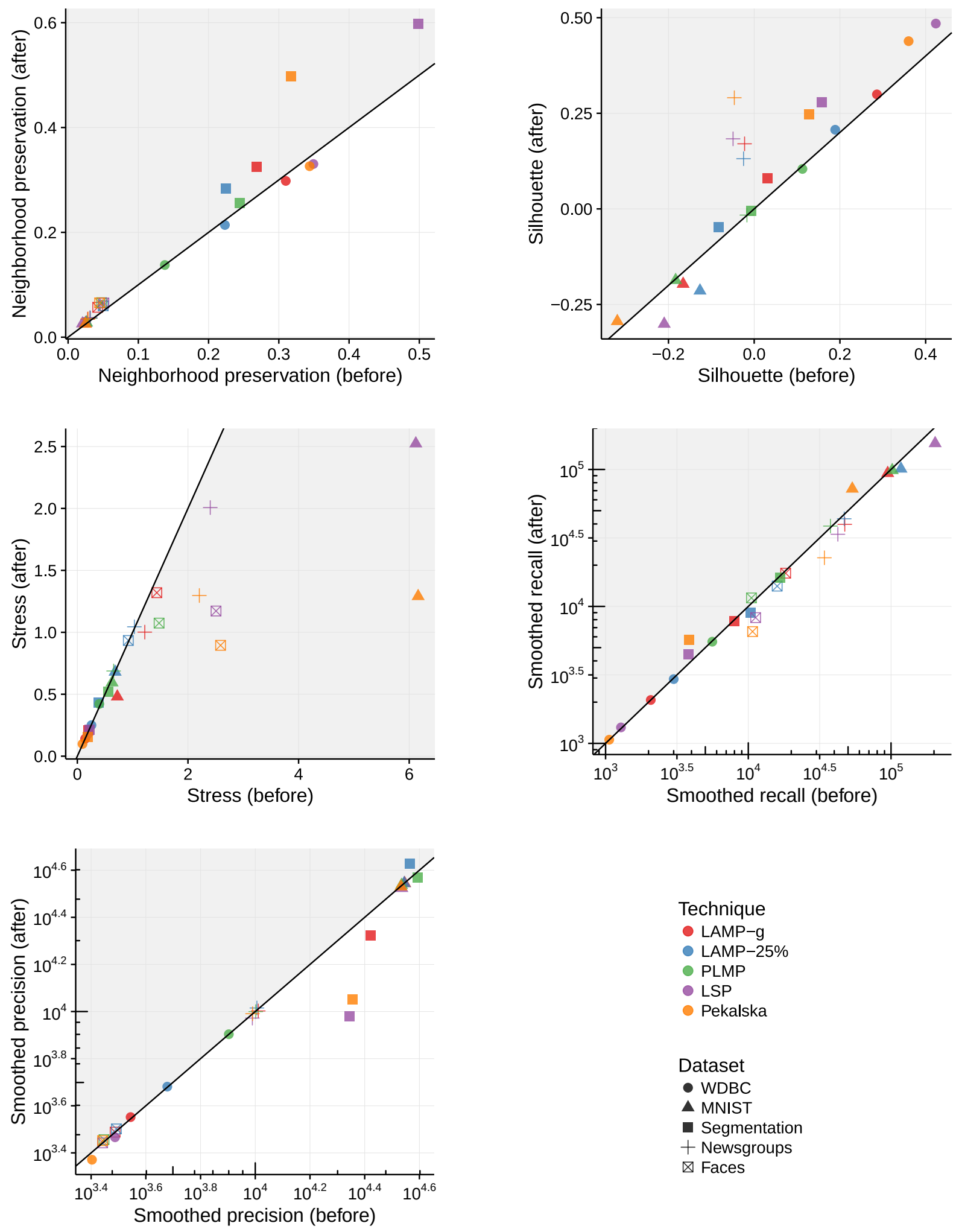

Source: Research data. 
Figure 19 - Line plots of $95 \%$ confidence intervals for the difference between a measure after manipulation and before. Since the FACES dataset has no labels, it is not shown in the silhouette coefficient plot.
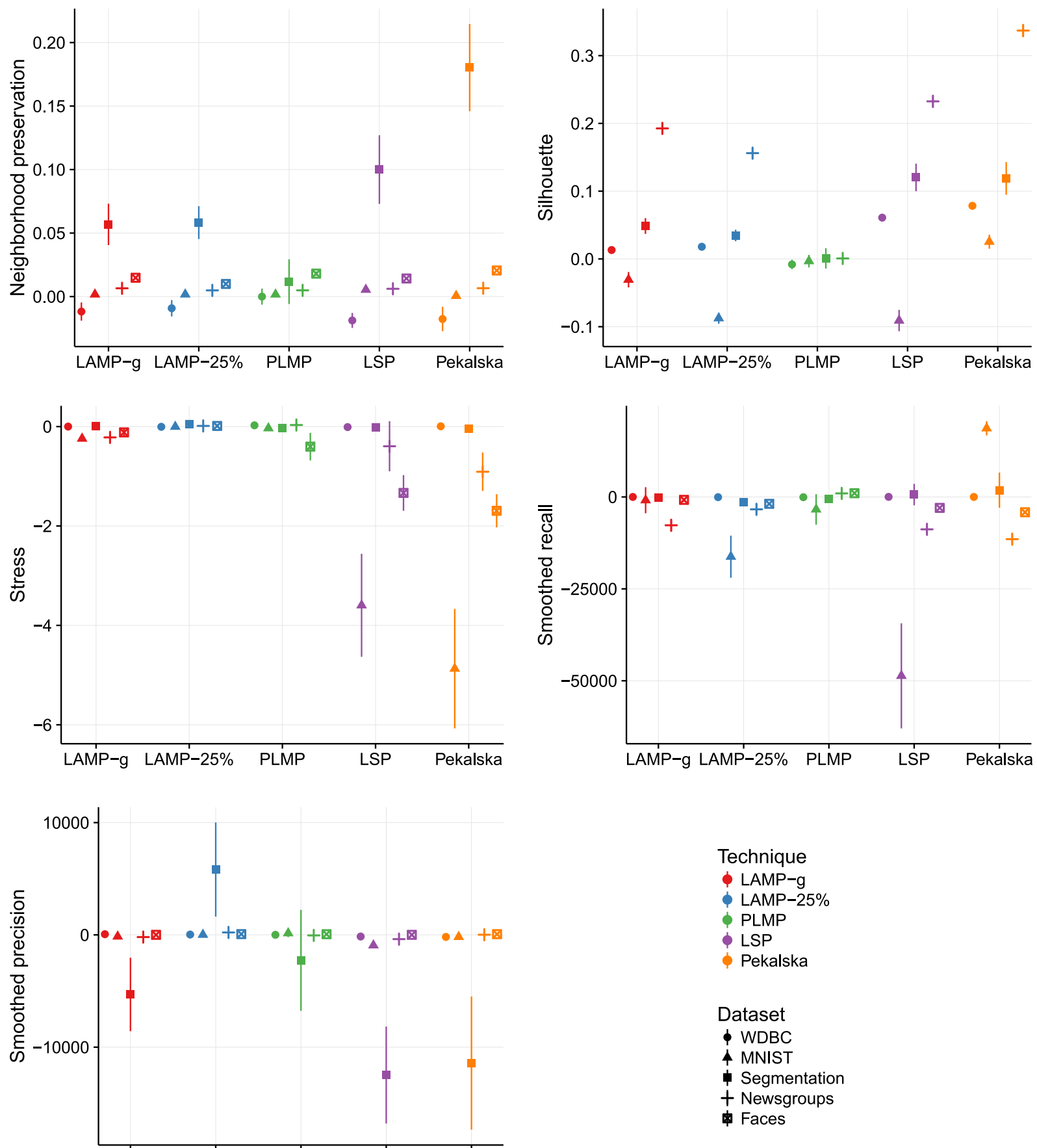

LAMP-g LAMP-25\% PLMP LSP Pekalska

Source: Research data. 
Figure 20 - The improvement in measures both in $\mathscr{Y}^{\prime}$ and $\mathscr{Y}$, starting from a randomly projected $\mathscr{Y}^{\prime}$ and then moving towards an improved placement according to a measure. The diagonal line $(y=x)$ is displayed as a reference for comparison between axes. Since the FACES dataset has no labels, it is not shown in the silhouette coefficient plot. The highlighted area denotes an improvement in $\mathscr{Y}$ with respect to $\mathscr{Y}^{\prime}$.
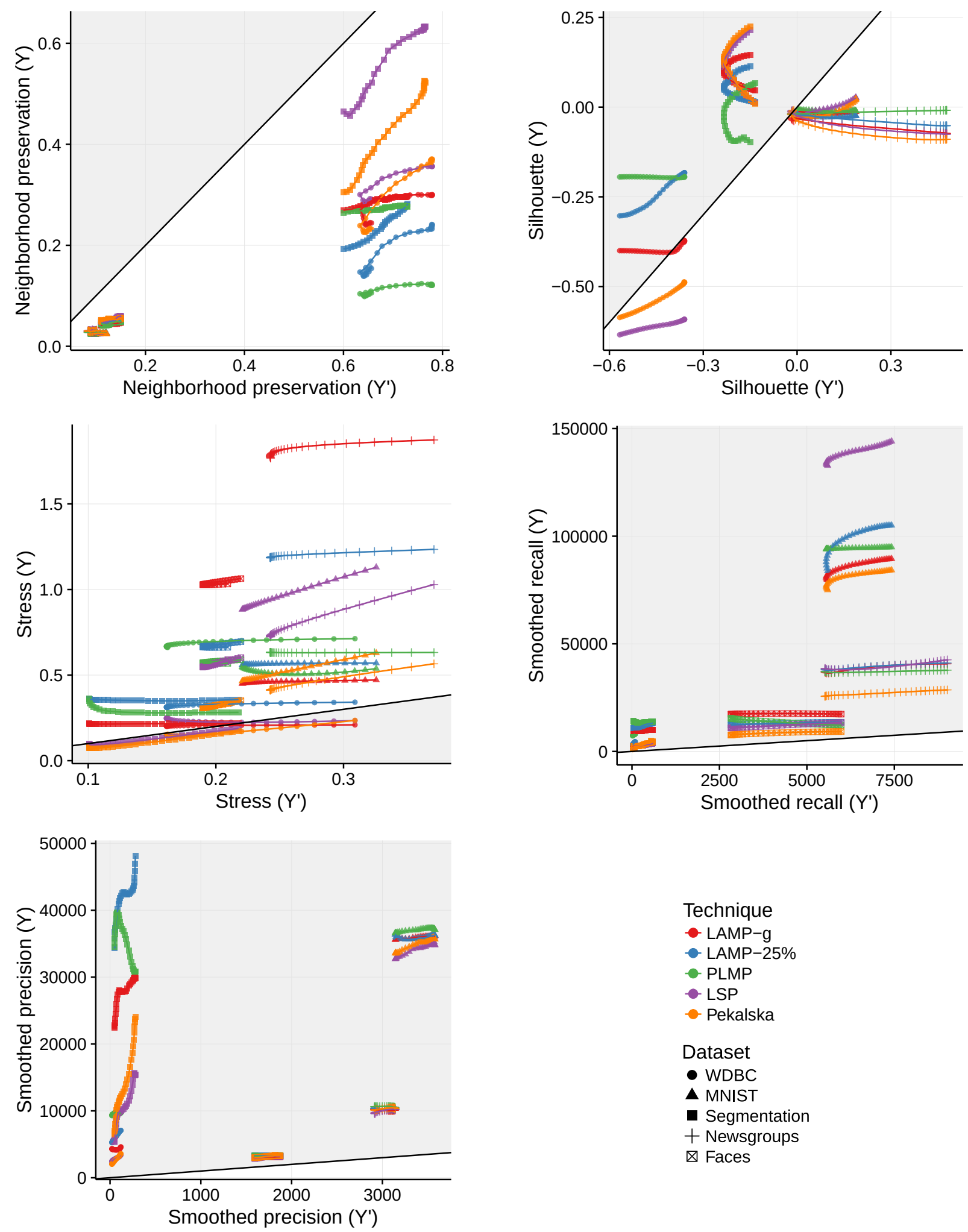

Technique

- LAMP-g

- LAMP-25\%

- PLMP

- LSP

- Pekalska

Dataset

- WDBC

A MNIST

- Segmentation

+ Newsgroups

$\nabla$ Faces

Source: Research data. 

CHAPTER

6

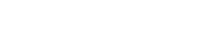

\section{CONCLUSIONS}

In this chapter we summarize the conclusions from Chapter 3 and Chapter 4, outlining the main contributions, limitations and some directions for future research.

This research is concerned with two different aspects of the same issue, which is understanding interactivity in multidimensional projections. Interactivity exploits the visual representation of complex data with our intuition in visual exploration, providing many analysis scenarios. From the users' point of view, interactivity is a way of incorporating our domain knowledge and hint the algorithm towards a mapping that overcomes some limitation in the data representation. From the perspective of the problem of mapping high-dimensional data to a low-dimensional representation, interactivity is one of the means of providing partial solutions to the algorithm.

\subsection{Contributions}

The contributions of this research are focused on the two discussed aspects of understanding interactivity. First, we designed visualizations that provide cues to explain the manipulation process. These cues serve the purpose of providing the necessary information to the user aiming at reducing the trial-and-error nature of interacting with a simple representation of high dimensional data. By designing a visualization for each step of the process, the user is guided by the additional information so that better decisions are made before attempting a manipulation. Moreover, these visualizations also emphasize and explain the changes caused by manipulation, another issue related to the high dimensionality of the data. While designed with a single technique in mind, visualizations such as those targeted at explaining changes are general enough to be reusable with other techniques.

Second, more contributions come from the empirical analysis of interaction in multidimensional projections. The definition of effectiveness that takes into account measurable influences caused by interaction, could be a useful guide for further research on the behavior of 
such techniques. Furthermore, we provide an experimental framework for assessing whether techniques are capable of effectively incorporating user manipulation into their computed mappings. Additionally, a series of experiments with this framework on several datasets and techniques were performed, demonstrating the behavior of many state of the art techniques.

From these results, we have significant evidence that LSP and Pekalska's technique are the most capable of ensuing effective manipulations. Moreover, the silhouette coefficient manipulation, despite ignoring the original dissimilarities to attain good representations of the original data labels, lead to one of the most effective manipulations. LSP, being a technique that positions instances inside the convex hull of their nearest neighbors' control points, is able to properly respect the positioning given by the automated manipulation procedures. Pekalska's technique, surprisingly, overcame others such as LAMP using only a number of local control points for each point. We believe that its original formulation, intended as an approximation of Sammon's Mapping, captures better than PLMP and LAMP the mapping from dissimilarities to $q$-dimensional coordinates. Thus, since both the stress optimization and the silhouette coefficient manipulation are directly interfering with this aspect, Pekalska's technique enables an effective manipulation in those cases.

\subsection{Limitations and future research}

Regarding the visualizations for guiding and explaining changes in interactions, the first notable drawback is the lack of a formal user study. We believe a formal user study could provide more solid evidence that the visualizations can be successfully used for their purposes, increasing the value of the contribution. Another limitation is that these visualizations are tailored to a specific technique, in this case LAMP. LAMP is cost-efficient enough to allow interactive manipulations while at the same time being capable of clearly determining what influence control points have on the positioning with respect to any point. Consequently, extending the visualizations to other techniques could be an interesting line of research, though a challenging one in this regard. Moreover, we believe an interesting line of further research could be related to predicting the results of manipulation. While our visualizations already provide a large portion of the information necessary to performing more precise manipulations, they cannot provide insight into the final layout. Approximations of, or even running LAMP on a limited number of control points, coupled with vector field visualization techniques could further reduce trial and error in the interactive process.

As for the framework for assessing the effectiveness of manipulation, it is limited in essence by being empirical. Theoretical studies, though significantly more difficult to conduct, would not only bring forth more reliable results, but could also lead to insights into better interactivity or even new techniques. Furthermore, we do not consider the addition or removal of control points, that is, choosing or removing some regular points as control points, could help 
the procedures achieve better improvements. Investigating these effects could also lead to an interesting study.

Another possible line of research would involve integrating the manipulation procedures into the visualizations as a guide for future manipulations. The user would focus on one aspect of the map and could then see what kinds of changes would occur with each type of measure being improved. 



\section{BIBLIOGRAPHY}

ACHLIOPTAS, D. Database-friendly Random Projections. In: Proceedings of the Twentieth ACM SIGMOD-SIGACT-SIGART Symposium on Principles of Database Systems. New York, NY, USA: ACM, 2001. (PODS '01), p. 274-281. ISBN 1-58113-361-8. Cited on page 67.

ANDREWS, K.; KIENREICH, W.; SABOL, V.; BECKER, J.; DROSCHL, G.; KAPPE, F.; GRANITZER, M.; AUER, P.; TOCHTERMANN, K. The InfoSky visual explorer: exploiting hierarchical structure and document similarities. Information Visualization, Palgrave Macmillan, v. 1, n. 3/4, p. 166-181, 2002. ISSN 1473-8716. Cited 2 times on pages 17 and 18.

ASIMOV, D. The grand tour: A tool for viewing multidimensional data. v. 6, n. 1, p. 128-143, 1985. ISSN 0196-5204. Cited 2 times on pages 39 and 40.

BAEZA-YATES, R.; RIBEIRO-NETO, B. Modern information retrieval: The concepts and technology behind search. In: [S.1.]: Addison Wesley, 2011. cap. Retrieval Evaluation. ISBN 9780321416919. Cited on page 62.

BROWN, E.; LIU, J.; BRODLEY, C.; CHANG, R. Dis-function: Learning distance functions interactively. In: 2012 IEEE Conference on Visual Analytics Science and Technology (VAST). [S.1.: s.n.], 2012. p. 83-92. Cited 2 times on pages 37 and 38.

CHALMERS, M. A linear iteration time layout algorithm for visualising high-dimensional data. In: Visualization '96. Proceedings. [S.1.: s.n.], 1996. p. 127-131. Cited 2 times on pages 28 and 34.

CHEN, Y.; WANG, L.; DONG, M.; HUA, J. Exemplar-based visualization of large document corpus (infovis2009-1115). IEEE Transactions on Visualization and Computer Graphics, IEEE Computer Society, Los Alamitos, CA, USA, v. 15, n. 6, p. 1161-1168, 2009. ISSN 1077-2626. Cited 2 times on pages 17 and 18.

COOK, D.; BUJA, A.; CABRERA, J.; HURLEY, C. Grand tour and projection pursuit. v. 4, p. 155-172, 1995. Cited 2 times on pages 26 and 40.

COX, T. F.; COX, M. A. A. Multidimensional Scaling. Second. [S.1.]: CRC Press, 2000. ISBN 9781420036121. Cited 2 times on pages 24 and 25.

ELMQVIST, N.; DRAGICEVIC, P.; FEKETE, J. Rolling the dice: Multidimensional visual exploration using scatterplot matrix navigation. v. 14, n. 6, p. 1539-1148, 2008. ISSN 1077-2626. Cited 3 times on pages 11, 41, and 42.

FADEL, S. G.; FATORE, F. M.; DUARTE, F. S. L. G.; PAULOVICH, F. V. LoCH: A neighborhood-based multidimensional projection technique for high-dimensional sparse spaces. Neurocomputing, v. 150, Part B, p. 546-556, fev. 2015. ISSN 0925-2312. Cited 5 times on pages $29,57,58,64$, and 67. 
FRIEDMAN, J.; TUKEY, J. A projection pursuit algorithm for exploratory data analysis. C-23, n. 9, p. 881-890, 1974. ISSN 0018-9340. Cited on page 26.

GOWER, J. C.; DIJKSTERHUIS, G. B. Procrustes Problems. [S.1.]: Oxford Statistical Science Series 30, 2004. ISBN 978-0-19-851058-1. Cited on page 35.

GUYON, I.; ELISSEEFF, A. An introduction to variable and feature selection. v. 3, p. 1158-1182, 2003. ISSN 1532-4435. Cited on page 22.

HEER, J.; SHNEIDERMAN, B. Interactive dynamics for visual analysis. v. 10, n. 2, p. 30:3030:55, 2012. ISSN 1542-7730. Cited on page 17.

HINTON, G.; ROWEIS, S. Stochastic neighbor embedding. In: BECKER, S.; THRUN, S.; OBERMAYER, K. (Ed.). Advances in Neural Information Processing Systems 15. [S.1.]: MIT Press, 2003. p. 857-864. Cited on page 30.

HUBER, P. J. Projection pursuit. v. 13, n. 2, p. 435-475, 1985. ISSN 0090-5364. Cited 2 times on pages 26 and 40.

HURTER, C.; TAYLOR, R.; CARPENDALE, S.; TELEA, A. Color Tunneling: Interactive Exploration and Selection in Volumetric Datasets. In: 2014 IEEE Pacific Visualization Symposium. [S.1.]: IEEE, 2014. p. 225-232. ISBN 978-1-4799-2874-3. Cited on page 53.

II, J. D.; ANDERSON, E. W.; NONATO, L. G.; SILVA, C. T. Interactive vector field feature identification. IEEE Transactions on Visualization and Computer Graphics, IEEE Educational Activities Department, Piscataway, NJ, USA, v. 16, n. 6, p. 1560-1568, nov. 2010. ISSN 1077-2626. Cited on page 17.

INGRAM, S.; MUNZNER, T.; OLANO, M. Glimmer: Multilevel MDS on the GPU. v. 15, n. 2, p. 249-261, 2009. ISSN 1077-2626. Cited on page 29.

JOIA, P.; PAULOVICH, F. V.; COIMBRA, D.; CUMINATO, J. A.; NONATO, L. G. Local affine multidimensional projection. v. 17, n. 12, p. 2563-2571, 2011. ISSN 1077-2626. Cited 5 times on pages $18,35,36,57$, and 58 .

JOLLIFFE, I. Principal component analysis. In: Encyclopedia of Statistics in Behavioral Science. [S.1.]: John Wiley \& Sons, Ltd, 2005. p. 1580-1584. ISBN 9780470013199. Cited 2 times on pages 22 and 23.

KAUFMAN, L.; ROUSSEEUW, P. Clustering by means of medoids. Statistical Data Analysis Based on the L1-Norm and Related Methods, p. North--Holland, 1987. Cited on page 58.

KEIM, D. Information visualization and visual data mining. v. 8, n. 1, p. 1-8, 2002. ISSN 1077-2626. Cited 2 times on pages 21 and 39.

KEIM, D.; KOHLHAMMER, J.; ELLIS, G.; MANSMANN, F. Mastering the Information Age: Solving Problems with Visual Analytics. [S.1.]: Florian Mansmann, 2010. ISBN 9783905673777. Cited on page 17.

LECUN, Y.; BOTTOU, L.; BENGIO, Y.; HAFFNER, P. Gradient-based learning applied to document recognition. Proceedings of the IEEE, IEEE, v. 86, n. 11, p. 2278-2324, 1998. ISSN 00189219. Cited on page 66. 
LESPINATS, S.; AUPETIT, M. CheckViz: Sanity check and topological clues for linear and non-linear mappings. v. 30, n. 1, p. 113-125, 2011. ISSN 1467-8659. Cited on page 47.

LICHMAN, M. UCI Machine Learning Repository. 2013. Disponível em: <http://archive.ics. uci.edu/ml>. Cited on page 66.

LIU, Y. Distance Metric Learning: A Comprehensive Survey. [S.1.], 2006. Cited on page 37.

MAATEN, L. van der; HINTON, G. Visualizing data using t-SNE. v. 9, p. 2579-2605, 2008. ISSN 1532-4435. Cited on page 31.

MAATEN, L. van der; POSTMA, E.; HERIK, J. van den. Dimensionality Reduction: A Comparative Review. [S.1.], 2009. Cited 4 times on pages 22, 23, 26, and 29.

MAMANI, G. M. H.; FATORE, F. M.; NONATO, L. G.; PAULOVICH, F. V. User-driven feature space transformation. v. 32, n. 3, p. 291-299, 2013. ISSN 1467-8659. Cited 5 times on pages $11,18,38,39$, and 57.

MARTINS, R. M.; COIMBRA, D. B.; MINGHIM, R.; TELEA, A. C. Visual analysis of dimensionality reduction quality for parameterized projections. Computers \& Graphics, v. 41, p. 26-42, jun 2014. ISSN 0097-8493. Cited 3 times on pages 11, 47, and 59.

MORRISON, A.; ROSS, G.; CHALMERS, M. A hybrid layout algorithm for sub-quadratic multidimensional scaling. In: Proceedings of the IEEE Symposium on Information Visualization (InfoVis'02). [S.1.]: IEEE Computer Society, 2002. (INFOVIS '02), p. 152-. ISBN 0-7695-1751-X. Cited 2 times on pages 29 and 34.

PAULOVICH, F. V.; ELER, D. M.; POCO, J.; BOTHA, C. P.; MINGHIM, R.; NONATO, L. G. Piecewise laplacian-based projection for interactive data exploration and organization. v. 30, n. 3, p. 1091-1100, 2011. ISSN 1467-8659. Cited on page 34.

PAULOVICH, F. V.; MAKI, R. M.; OLIVEIRA, M. C. F.; COLHONE, M. C.; SANTOS, F. R.; MIGLIACCIO, V.; CIANCAGLINI, P.; PEREZ, K. R.; STABELI, R. G.; PERINOTO, A. C.; OLIVEIRA OSVALDO N., J.; COLOTTO, V. Z. Using multidimensional projection techniques for reaching a high distinguishing ability in biosensing. Analytical and Bioanalytical Chemistry, Springer-Verlag, v. 400, n. 4, p. 1153-1159, 2011. ISSN 1618-2642. Cited on page 18.

PAULOVICH, F. V.; MINGHIM, R. HiPP: A Novel Hierarchical Point Placement Strategy and its Application to the Exploration of Document Collections. IEEE Transactions on Visualization and Computer Graphics, IEEE, v. 14, n. 6, p. 1229-1236, nov 2008. ISSN 1077-2626. Cited 2 times on pages 59 and 62 .

PAULOVICH, F. V.; MORAES, M. L.; MAKI, R. M.; FERREIRA, M.; JR., O. N. O.; OLIVEIRA, M. C. F. de. Information visualization techniques for sensing and biosensing. Analyst, The Royal Society of Chemistry, v. 136, p. 1344-1350, 2011. Cited on page 18.

PAULOVICH, F. V.; NONATO, L.; MINGHIM, R.; LEVKOWITZ, H. Least square projection: A fast high-precision multidimensional projection technique and its application to document mapping. v. 14, n. 3, p. 564-575, 2008. ISSN 1077-2626. Cited 7 times on pages 17, 18, 32, 33, 57,58 , and 67. 
PAULOVICH, F. V.; SILVA, C. T.; NONATO, L. G. Two-phase mapping for projecting massive data sets. v. 16, n. 6, p. 1281-1290, 2010. ISSN 1077-2626. Cited 5 times on pages 18, 34, 57, 58 , and 68 .

. User-centered multidimensional projection techniques. v. 14, n. 4, p. 74-81, 2012. ISSN 1521-9615. Cited on page 18.

PEKALSKA, E.; RIDDER, D. de; DUIN, R. P.; KRAAIJVELD, M. A. A new method of generalizing sammon mapping with application to algorithm speed-up. In: . [S.l.: s.n.], 1999. p. 221-228. Cited 3 times on pages 18, 27, and 28.

PUDIL, P.; HOVOVICOVA, J. Novel methods for subset selection with respect to problem knowledge. v. 13, n. 2, p. 66-74, 1998. ISSN 1094-7167. Cited on page 22.

ROBERTSON, G.; FERNANDEZ, R.; FISHER, D.; LEE, B.; STASKO, J. Effectiveness of Animation in Trend Visualization. IEEE Transactions on Visualization and Computer Graphics, IEEE, v. 14, n. 6, p. 1325-1332, 2008. Cited on page 53.

ROUSSEEUW, P. Silhouettes: a graphical aid to the interpretation and validation of cluster analysis. Journal of Computational and Applied Mathematics, Elsevier Science Publishers B. V., Amsterdam, The Netherlands, The Netherlands, v. 20, n. 1, p. 53-65, nov. 1987. ISSN 0377-0427. Cited 2 times on pages 59 and 61.

SAMMON, J. W. A nonlinear mapping for data structure analysis. v. 18, n. 5, p. 401-409, 1969. ISSN 0018-9340. Cited 3 times on pages 27, 59, and 62.

SCHAEFER, M.; ZHANG, L.; SCHRECK, T.; TATU, A.; LEE, J. A.; VERLEYSEN, M.; KEIM, D. A. Improving projection-based data analysis by feature space transformations. In: . [S.l.: s.n.], 2013. v. 8654. Cited on page 64.

SHNEIDERMAN, B. The eyes have it: a task by data type taxonomy for information visualizations. In: , IEEE Symposium on Visual Languages, 1996. Proceedings. [S.1.: s.n.], 1996. p. 336-343. Cited 2 times on pages 17 and 21.

SILVA, V. d.; TENENBAUM, J. B. Sparse multidimensional scaling using landmark points. [S.1.], 2004. Cited 2 times on pages 25 and 32.

STAHNKE, J.; DORK, M.; MULLER, B.; THOM, A. Probing Projections: Interaction Techniques for Interpreting Arrangements and Errors of Dimensionality Reductions. IEEE Transactions on Visualization and Computer Graphics, v. 22, n. 1, p. 629-638, jan 2016. ISSN 1077-2626. Cited on page 47.

SUN, G.-D.; WU, Y.-C.; LIANG, R.-H.; LIU, S.-X. A survey of visual analytics techniques and applications: State-of-the-art research and future challenges. v. 28, n. 5, p. 852-867, 2013. ISSN 1000-9000, 1860-4749. Cited on page 17.

TEJADA, E.; MINGHIM, R.; NONATO, L. G. On improved projection techniques to support visual exploration of multi-dimensional data sets. v. 2, n. 4, p. 218-231, 2003. ISSN 1473-8716, 1473-8724. Cited on page 29.

TENENBAUM, J. B.; SILVA, V. de; LANGFORD, J. C. A global geometric framework for nonlinear dimensionality reduction. Science (New York, N.Y.), American Association for the Advancement of Science, v. 290, n. 5500, p. 2319-23, dec 2000. ISSN 0036-8075. Cited on page 66 . 
THEUS, M. High-dimensional data visualization. In: Handbook of Data Visualization. [S.1.]: Springer Berlin Heidelberg, 2008, (Springer Handbooks Comp.Statistics). p. 151-178. ISBN 978-3-540-33037-0. Cited on page 40.

VENNA, J.; PELTONEN, J.; NYBO, K.; AIDOS, H.; KASKI, S. Information Retrieval Perspective to Nonlinear Dimensionality Reduction for Data Visualization. Journal of Machine Learning Research, Volume 11, p. 451-490, feb 2010. Cited 5 times on pages 11, 31, 59, 63, and 67.

WISE, J. A. The ecological approach to text visualization. Journal of the American Society for Information Science, John Wiley \& Sons, Inc., New York, NY, USA, v. 50, n. 13, p. 1224-1233, 1999. ISSN 0002-8231. Cited 2 times on pages 17 and 18.

ZWAN, M. van der; CODREANU, V.; TELEA, A. CUBu : Universal real-time bundling for large graphs. IEEE Transactions on Visualization and Computer Graphics, 2016. Cited on page 50 . 

For convenience, this appendix summarizes and explains the notation used throughout this document. In general, scalars are lowercase letters, vectors are bold lowercase letters, matrices are uppercase letters and sets use caligraphic uppercase letters. It is assumed that vectors are column vectors, unless otherwise stated. The following list contains often used symbols and their meaning.

$\mathbb{R}_{0}^{+} \quad$ The set $\{x \in \mathbb{R}: x \geq 0\}=[0, \infty)$.

$n \quad$ The dataset size; number of observations of each variable.

$p \quad$ Original number of variables; number of dimensions; dimensionality.

$q \quad$ Number of variables after dimensionality reduction or data transformation. Usually assumed to be 2 or 3 .

$\mathscr{X} \quad$ A set of tuples in $\mathbb{R}^{p}$. Represents $n$ observations of $p$ variables. Also called a dataset.

$\mathscr{Y} \quad$ A set of tuples in $\mathbb{R}^{q}$. Represents $\mathscr{X}$ after dimensionality reduction.

$X, Y$ Matrix representation of $\mathscr{X}$ and $\mathscr{Y}$, respectively.

$\mathbf{x}_{i} \quad$ Both a tuple of $\mathscr{X}$ or a row of $X$, whichever is contextually more appropriate. Also called a datum, instance or sample.

$\mathbf{y}_{i} \quad$ Both a tuple of $\mathscr{Y}$ or a row of $Y$, whichever is contextually more appropriate.

$\delta \quad$ A dissimilarity function. Usually defined as $\delta: \mathbb{R}^{p} \times \mathbb{R}^{p} \rightarrow \mathbb{R}_{0}^{+}$, unless otherwise stated.

$\delta_{i j} \quad$ The dissimilarity between objects $i$ and $j$; the value of $\delta\left(\mathbf{x}_{i}, \mathbf{x}_{j}\right)$.

$d_{i j} \quad$ The value of $\left\|\mathbf{y}_{i}-\mathbf{y}_{j}\right\|$. 
$[M]_{i j}$ The element in row $i$ and column $j$ of matrix $M$. 


\section{DATA PREPARATION AND PREPROCESSING}

This appendix summarizes the steps in preparing the datasets for the experiments described in Chapter 5. We performed normalizations and the other steps whenever either the dataset authors recommended them or we observed that it resulted in layouts subjectively more similar in structure, up to what is possible with different techniques. Moreover, all duplicate rows found in datasets were removed. Techniques such as Sammon's Mapping (subsection 2.1.4), used in improving the stress measure, require this preprocessing step. Below we describe other steps performed specifically for each dataset, in no particular order.

FACES: This dataset went through a preliminary dimensionality reduction to 256 dimensions using PCA with whitening.

WDBC: For this dataset, we only perform standardization, subtracting the mean of each column from itself, and then diving the resulting column by its standard deviation.

SEGMENTATION: We removed the third column from this dataset as all values were equal. We then performed standardization on the remaining columns (the removed column could not be standardized as all values were equal).

NEWSGROUPS: To transform this textual dataset into a multidimensional dataset, we used the term frequency, inverse document frequency (TF-IDF) transformation using 500 randomly selected news articles of the following three topics: comp.graphics, misc.forsale and sci.med. The text was also preprocessed to remove english stopwords ${ }^{1}$. Terms with a document frequency of less than 0.01 were also ignored. All other parameters of the

1 We used the list from <http://snowball.tartarus.org/algorithms/english/stop.txt>, including the suggested stopwords at the end. 
method were left as-is from the Scikit-Learn ${ }^{2}$ software package. We then reduced the attained dimensionality to 512, using PCA with whitening.

MNIST: This dataset originally consists of 60,000 images. We randomly selected 1,000 images from the training set and 1,000 images from the test set. This resulted in some repeated images, which were then removed by our other preprocessing step, described earlier. The images were transformed into vectors by concatenating each row into the one above. Then, the dimensionality of the vectors was reduced to 512, using PCA with whitening. 